\title{
Patient-Specific Induced Pluripotent Stem Cell Models of Parkinson's Disease
}

\author{
Dissertation \\ for the award of the degree \\ "Doctor rerum naturalium" (Dr. rer. nat.) \\ of Georg-August-Universität Göttingen \\ at the Faculty of Biology \\ of the Georg-August University School of Science (GAUSS)
}

submitted by

Mei-Chih Liao

from Taichung, Taiwan

Göttingen, 2013 


\section{Thesis Committee:}

Prof. Dr. Ahmed Mansouri

Molecular Cell Differentiation Group, Max Planck Institute for Biophysical Chemistry, Göttingen

Prof. Dr. Ernst Wimmer

Department of Developmental Biology, Georg-August-Universität Göttingen

\section{Members of the Examination Board:}

Prof. Dr. Ahmed Mansouri

Molecular Cell Differentiation Group, Max Planck Institute for Biophysical Chemistry, Göttingen

Prof. Dr. Ernst Wimmer

Department of Developmental Biology, Georg-August-Universität Göttingen

\section{Further Members of the Examination Board:}

Prof. Dr. Andreas Wodarz

Department of Anatomy and Cell Biology, Georg-August-Universität Göttingen

Dr. Roland Dosch

Department of Developmental Biochemistry, Georg-August-Universität Göttingen

Prof. Dr. Walter Paulus

Department of Clinical Neurophysiology, Universitätsmedizin Göttingen

Prof. Dr. Reinhard Schuh

Department of Molecular Developmental Biology, Max Planck Institute for Biophysical Chemistry, Göttingen 


\section{Declaration}

I hereby declare that this thesis "Patient-Specific Induced Pluripotent Stem Cell Models of Parkinson's Disease" has been written independently, with no other aids than those cited.

Mei-Chih Liao

Göttingen, August 2013 


\section{Acknowledgements}

When it comes to convey my acknowledgements for completion of this doctoral dissertation, I have lots of words and thoughts in my mind. However, I would apologize that I could not write them down word by word.

I would like to express my sincere appreciation towards Prof. Ahmed Mnsouri. Under his supervision, I got the opportunity to work in this quite interesting field of stem cell biology and continuous support. Although almost everything was new to me, I was encouraged to face challenges.

I also would love to thank Prof. Ernst Wimmer. As the other thesis supervisor, his advice and suggestions led me on the right way of doing the research.

In this work, many people contributed their efforts to it. Prof. Claudia Trenkwalder and Dr. Brit Mollenhauer, they were very kind to recruit PD patients for conducting the starting material for the project. I am very grateful to Prof. Ralf Dressel for the evaluation of teratoma formation. His patience and support gave me a lot of flexibility for doing work. Patrick Probst and Sigurd-Günter Hille, they helped me for those MolBio cloning and sequencing. Prof. Stefan Jakobs and Dr. Susann Kummer, I very appreciated them joining this project and devoting their aspirations. In particular, Susann she did a great work for phenotypic assay of mDA neurons. She also inspired and encouraged me to move forward to the further work.

During these 4 years as a doctoral student, I was very appreciated that I worked in the Molecular Cell Differentiation group and Department of Molecular Cell Biology. Thomas Schulz and Sharif Mahsur, they provided me many supports on cell culture. Since I spent most of time on cell culture, they are indeed important to me!! Dr. Christian Krug and Dr. Tamara Rabe, they are much more knowledgeable about the development of dopaminergic neurons. They are always nice to give me advice and help when I was lost in the maze of complicated brain. Dr. Zeeshan Ahmad, Farnaz Shamsi, and Dr. Hélène Foussard, I am very grateful to work with you, sharing the experience and life moments. Especially, they are patient to my occasional hyper. XD Thanks to all former and current members in the department for the inspiring and nice working environment.

And, but not the end, my father \& mother are my biggest support and always stand there for me. I wish them always keep healthy and happy. 


\section{Contents}

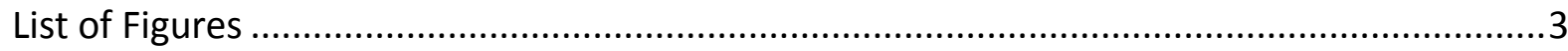

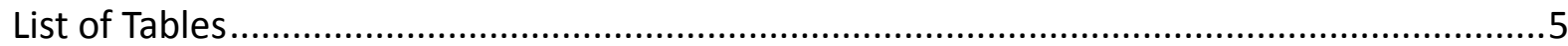

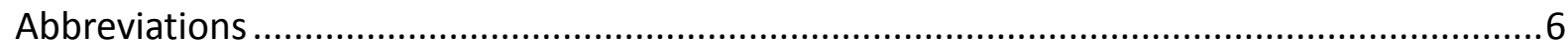

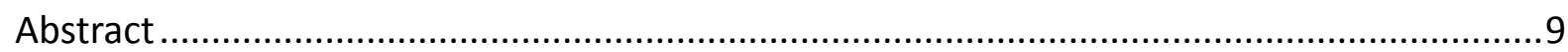

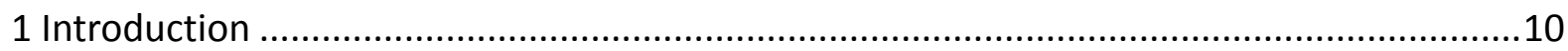

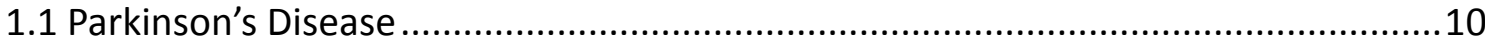

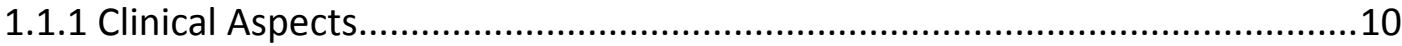

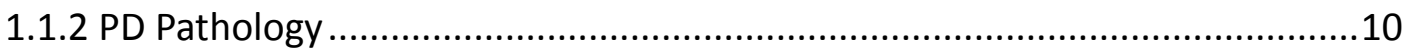

1.1.3 PD Etiology and Environmental Contribution ..........................................12

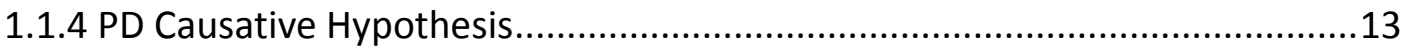

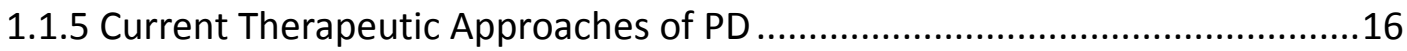

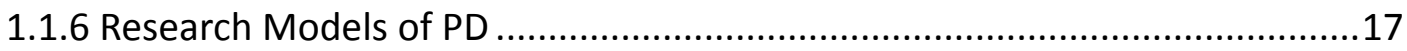

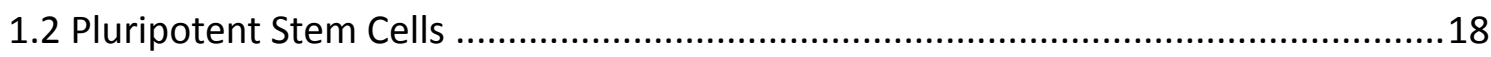

1.2.1 Human Embryonic Stem Cell ...................................................................18

1.2.2 Human Induced Pluripotent Stem Cell ....................................................19

1.2.3 Cloned Human Embryonic Stem Cell .........................................................21

1.3 Derivation of Dopaminergic Neurons from Human Pluripotent Stem Cell ...............24

1.4 Parkinson's Disease and Human Induced Pluripotent Stem Cell ...............................25

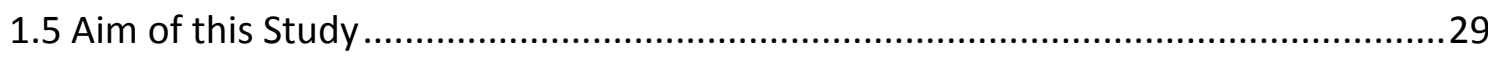

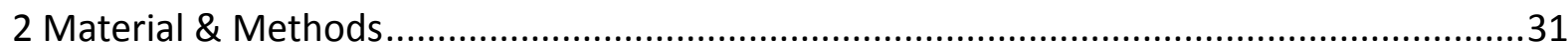

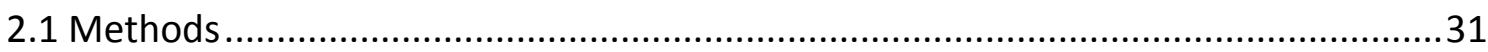

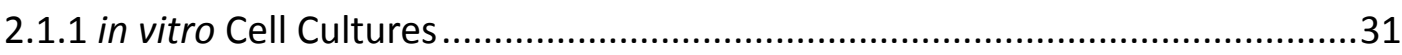

2.1.2 Biochemistry \& Molecular Biology Methods ...............................................36

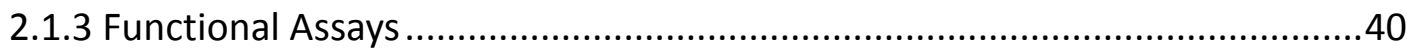

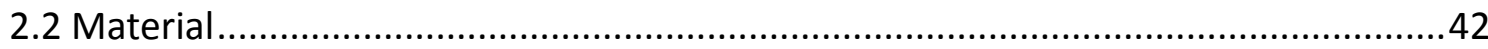

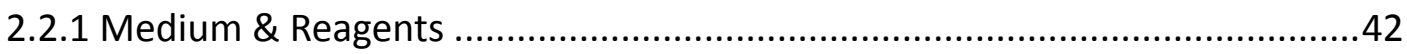

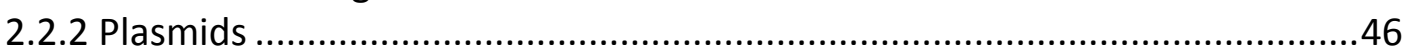

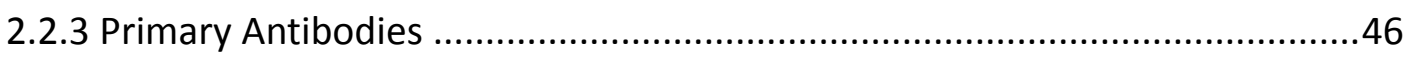

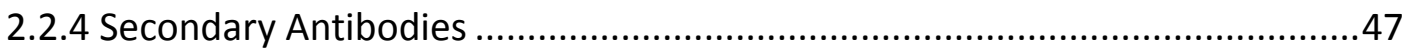

2.2.5 PCR Primers used for Cre Excision .............................................................48

2.2.6 PCR Primers used for Fingerprinting ..........................................................48

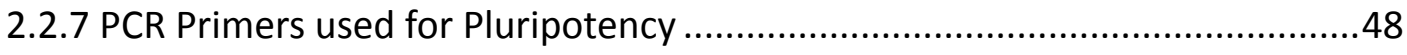

2.2.8 PCR Primers used for Bisulfite OCT4 Promoter ..........................................49

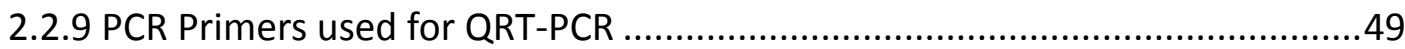

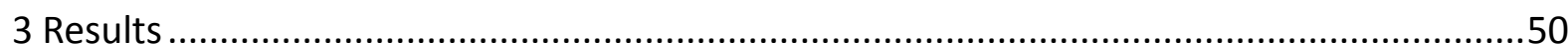

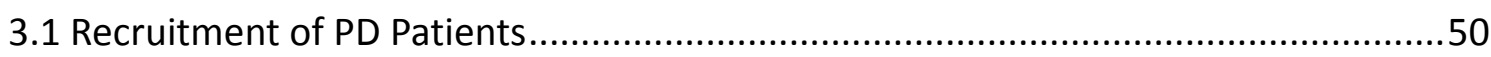

3.2 Generation of PD Patient Specific Induced Pluripotent Stem Cells ..........................51

3.2.1 Reprogramming of PD patients' Fibroblasts to Pluripotent States .................51

3.2.2 Excision of Transgene from Host Genome of Human ES-like Clones by

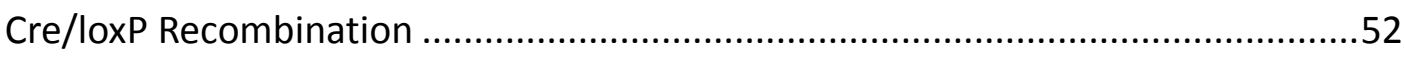

3.2.3 Characterization of PD Patient Specific hiPSC Lines ....................................56

3.3 Derivation of Neural Precursors from Control/PD-hiPSCs ........................................67

3.3.1 Direct Differentiation of Neural Precursors form Control and PD hiPSCs.......67 
3.3.2 Generation of dopaminergic neurons from Control/PD-hiPSC derived Neural

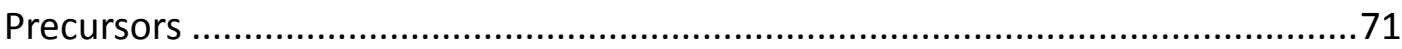

3.3.3 Transcriptome Analysis of Control/PD-hiPSC Derived Neural Precursors ......73 3.4 Derivation of Floor Plated-based Differentiated mDA Neurons from Conrtol/PD-hiPSCs .78

3.4.1 Direct Differentiation of midbrain Dopaminergic Neurons from Control/PD-hiPSCs .78

3.4.2 Electrophysiological Analysis of FP-based Control/PD-hiPSC Derived mDA

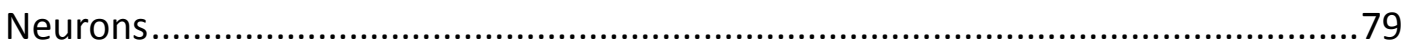

3.4.3 PD-hiPSC Derived mDA Neurons were Sensitive to CCCP-induced

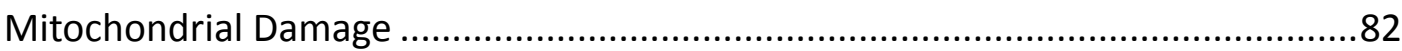

3.4.4 FGF20 Might Counteract the Mitochondrial Damage Induced by CCCP ........84

3.4.5 FGF20 Might Reduce the Excess Intracellular Reactive Oxygen Species (ROS)

.86

4 Discussion .88

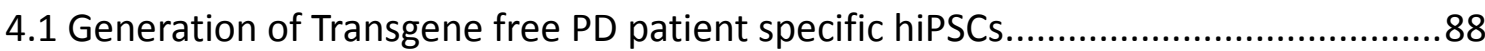

4.2 Derivation of Control/PD-hiPSC Neural Precursors and Transcriptome Analysis ......90 4.3 Direct differentiation of midbrain Dopaminergic (mDA) Neurons from

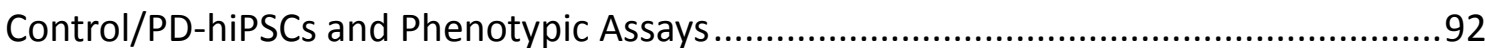

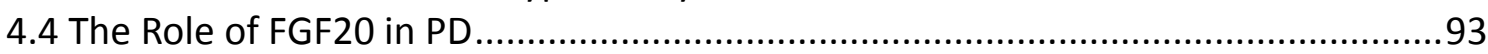

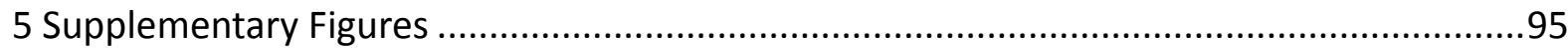

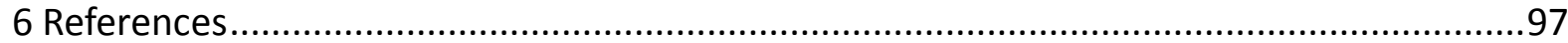




\section{List of Figures}

Figure 1.1: Brain Regions Affected by Parkinosn's Disease...................................................11

Figure 1.2: Micrographs of Mesencephalon Parkinson's Disease..............................................12

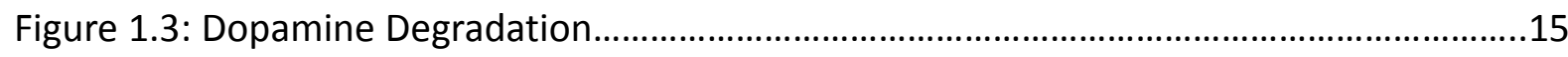

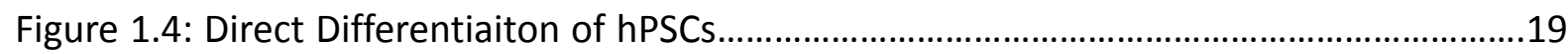

Figure 1.5: hiPSC Derivation, Differentiation, and Application..............................................21

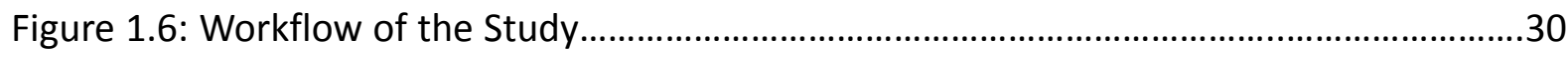

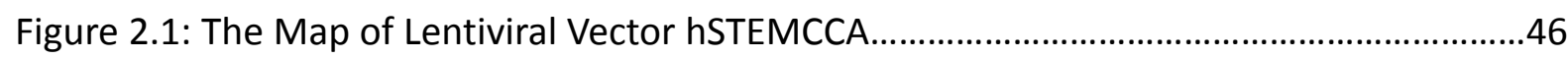

Figure 3.1 Generation of Human iPSC by a Lentiviral hSTEMCCA Vector.................................53

Figure 3.2: Cell Morphology of Human Skin Fibroblasts and Generated hiPSC Clones............54

Figure 3.3: Screen of Transgene-free hiPSC Clones by PCR and Southern Blot......................55

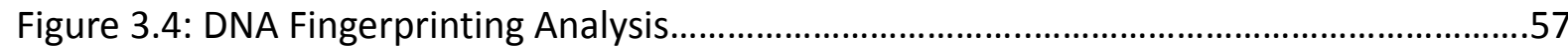

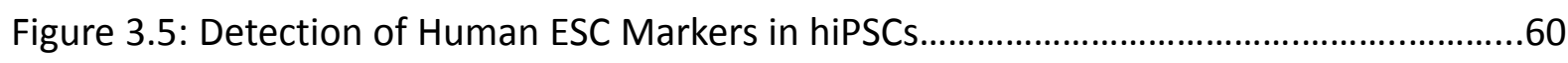

Figure 3.6: Methylation Status of OCT4 Promoter..................................................................61

Figure 3.7: in vitro and in vivo Differentiation of hiPSCs........................................................66

Figure 3.8: Flow Chart of Derivation of Neural Precursors.......................................................68

Figure 3.9: Neural Rosettes Structures in Neural Inductions...................................................69

Figure 3.10: Derivation of Neural precursors from Control/PD-hiPSCs Neural

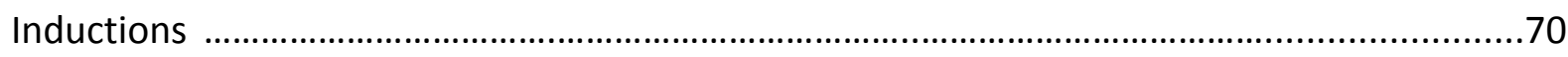

Figure 3.11: Dopamine Neuron Differentiation from PD-hiPSC derived Neural

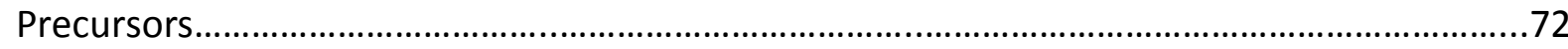

Figure 3.12: Comparative Transcriptome Analysis of Control/PD-hiPSC derived Neural

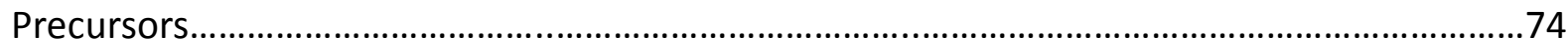

Figure 3.13: Functional Annotation Clustering by KEGG Pathway...........................................75

Figure 3.14: Expression Level of Genes of Susceptibility to Parkinson's Disease......................77

Figure 3.15: Flow Chart of FP-based Differentiation of Midbrain Dopaminergic

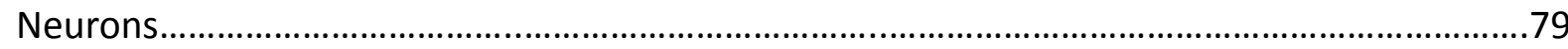

Figure 3.16: Induction and Maturation of hiPSC derived mDA Neurons...................................80

Figure 3.17: Electrophysiological Analyses of hiPSC Derived mDA Neurons..............................81

Figure 3.18: CCCP induced Mitochondrial Damage in hiPSC derived mDA Neurons................83 
Figure 3.19: Effect of FGF20 on CCCP Triggered Mitochondrial Damage in hiPSC-derived mDA

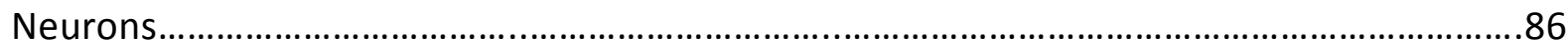

Figure 3.20: Effect of FGF2O on $\mathrm{H}_{2} \mathrm{O}_{2}$-induced Generation of Reactive Oxygen Species (ROS) in hiPSC-derived mDA Neurons .87

Figure 4.1: Various Cellular Stress Treatments on PD-hiPSC derived neural cells....................93

Figure 4.2: The Action of FGF20 on Dopaminergic Neurons..................................................94

Figure S1: KEGG Pathway Enrichment Analysis of Axon Guidance ..............................................95

Figure S2: Alkaline Phosphatase (ALP) Staining of hiPSC Colonies...........................................96 


\section{List of Tables}

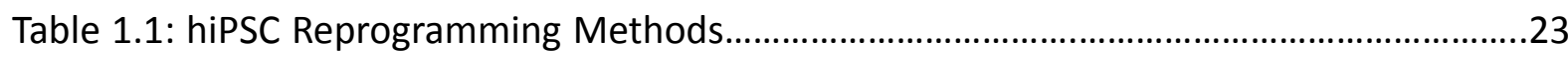

Table 1.2: Monogenic PD patients derived hiPSC and DA Neurons..............................................

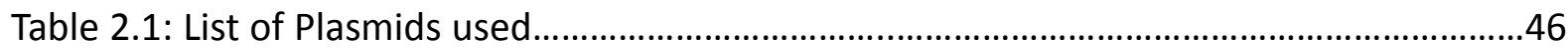

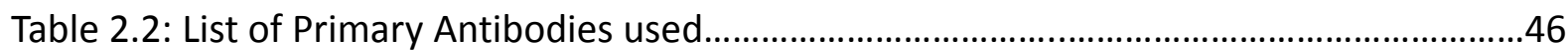

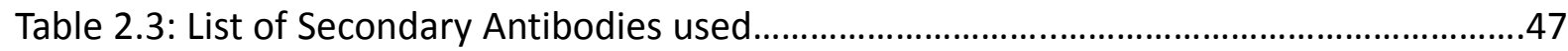

Table 2.4: List of PCR Primers for Cre Excision............................................................................48

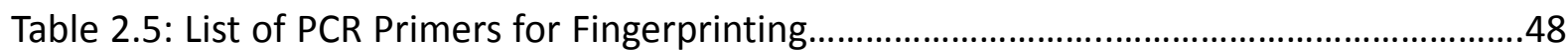

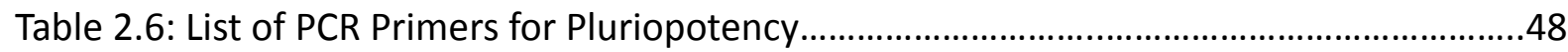

Table 2.7: List of PCR Primers for Bisulfite OCT4 Promoter........................................................49

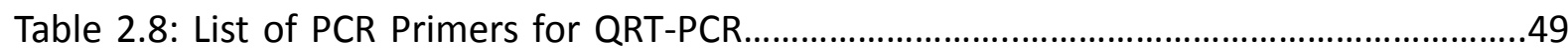

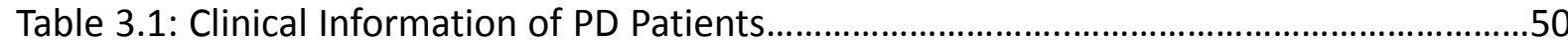

Table 3.2: Gene Ontology Analysis of Differential Gene Expression of Neuronal Precursors Annotated with the GO Term: Neuron Differentiation...............................................................76 


\section{Abbreviations}

$\begin{array}{ll}\text { ATP } & \text { adenosine triphosphate } \\ \text { BDNF } & \text { brain-derived neurotrophic factor } \\ \text { bFGF } & \text { basic Fibroblast growth factor } \\ \text { BSA } & \text { bovine serum albumin } \\ \text { cAMP } & \text { cyclic adenosine monophosphate } \\ \text { CCCP } & \text { carbonyl cyanide m-chlorophenyl hydrazone } \\ \text { cDNA } & \text { complementary deoxyribonucleic acid } \\ \text { CNS } & \text { central nervous system } \\ \text { DA } & \text { dopaminergic } \\ \text { DAPI } & \text { 4',6-Diamidino-2-phenylindole } \\ \text { DMEM } & \text { Dulbecco's modified Eagle's medium } \\ \text { DMSO } & \text { dimethyl sulfoxide } \\ \text { DNA } & \text { deoxyribonucleic acid } \\ \text { dNTP } & \text { deoxyribonucleotide triphosphate } \\ \text { EB } & \text { embryoid body } \\ \text { EDTA } & \text { Ethylenediaminetetraacetic acid } \\ \text { EPSC } & \text { excitatory postsynaptic current } \\ \text { ERK } & \text { extracellular signal-regulated protein kinases } \\ \text { ESC } & \text { embryonic stem cell } \\ \text { FACS } & \text { fluorescence-activated cell sorting } \\ \text { FCS } & \text { foetal calf serum } \\ \text { FGF2 } & \text { fibroblast growth factor } 2 \\ \text { FGF20 } & \text { fibroblast growth factor } 20 \\ \text { FGF8 } & \text { fibroblast growth factor } 8 \\ \text { FP } & \text { floor plate } \\ \text { GDNF } & \text { glial cell-derived neurotrophic factor } \\ \text { hO ontology } \\ \text { hour }\end{array}$




\begin{tabular}{|c|c|}
\hline hiPSC & human induced pluripotent stem cell \\
\hline iPSC & Induced pluripotent stem cell \\
\hline IRES & internal ribosome entry site \\
\hline KO & knockout \\
\hline KRS & knockout serum replacement media \\
\hline LRRK2 & Leucine-rich repeat kinase 2 \\
\hline LTR & long terminal repeat \\
\hline MAPK & mitogen-activated protein kinase \\
\hline $\mathrm{mDA}$ & midbrain dopaminergic \\
\hline MEF & mouse embryonic fibroblast \\
\hline MEF-CM & MEF conditioned media \\
\hline $\min$ & minute \\
\hline mRNA & messenger ribonucleic acid \\
\hline Na butyrate & sodium butyrate \\
\hline $\mathrm{P} / \mathrm{S}$ & penicillin/streptomycin \\
\hline PBS & Phosphate buffered saline \\
\hline PCR & Polymerase chain reaction \\
\hline PD & Parkinson's disease \\
\hline PDL & poly-D-lysine \\
\hline PFA & paraformaldehyde \\
\hline PNS & peripheral nervous system \\
\hline PSC & pluripotent stem cell \\
\hline qRT-PCR & quantitative reverse transcriptase PCR \\
\hline RNA & ribonucleic acid \\
\hline ROCK & rho-associated protein kinase \\
\hline ROS & reactive oxygen species \\
\hline RPM & revolutions per minute \\
\hline $\mathrm{rt}$ & room temperature \\
\hline RT-PCR & reverse transcriptase PCR \\
\hline SCNT & somatic cell nuclear transfer \\
\hline SD & standard deviation \\
\hline
\end{tabular}


SDS sodium dodecyl sulfate

SNpc substantia nigra pars compacta

SSEA stage specific embryonic antigen

SV40 simian virus 40 derived polyadenylation site

TGF 33 transforming growth factor 3

TH Tyrosine hydroxylase

TRA tumor rejection antigen

WPRE woodchuck hepatitis virus posttranscriptional regulatory element

wt wild-type 


\section{Abstract}

Parkinson's disease (PD) is one of the common neurodegenerative diseases. In particular, it affects the elderly and associates with the movement disorder. The hallmarks of PD are progressive loss of midbrain dopaminergic neurons and the appearance of Lewy bodies in the affected neurons. At present, the etiology of PD is not well understood and may reflect the interactions of environmental and genetic factors. The main hurdle in studying PD pathogenesis is the inaccessibility to the brain tissues from PD patients. Recent advances in cellular reprogramming now allow an alternative approach for investigating PD. PD patients' dermal fibroblasts can be converted to the pluripotent state, and further differentiated to the disease relevant cell type, dopaminergic neurons. Such PD affected neurons in culture represent a promising departure point for exploring the pathogenic factors of PD.

Here, we have generated disease specific human induced pluripotent stem cells (hiPSCs) from 3 individual idiopathic PD patients and 2 healthy persons' fibroblast. We applied a single lentiviral vector (hSTEMCCA-loxP) encoding OCT4, KLF4, SOX2, and C-MYC, and subsequently were able to generate the transgene-free hiPSCs using Cre-loxP recombination. The genomic constitutions of PD-hiPSCs were found identical to their parental fibroblasts by DNA fingerprinting. The characteristics of PD-hiPSCs resembled human embryonic stem cells (hESCs) displaying the expression of pluripotency marker genes, and the capability of differentiating into the three germ layers. Neural precursors were derived from PD-hiPSCs, and then examined by transcriptome analysis. Our results revealed some differentially expressed genes involved in axon guidance and neuron differentiation. Among those candidates, FGF2O was down regulated in PD patients. By Directed differentiation of floor-plate (FP) derived midbrain dopaminergic (mDA) neurons from hiPSCs, large population of specific midbrain dopaminergic neurons could be achieved. We exposed these control and PD specific hiPSCs derived mDA neurons to mitochondrial stress. PD-hiPSC derived mDA neurons were susceptible to CCCP treatments. Interestingly, FGF20 was able to counteract these cellular stresses. FGF20, therefore, might hold the capacity for dopaminergic neuroprotection. 


\section{Introduction}

\subsection{Parkinson's Disease}

Parkinson's disease (PD) is one of the most prevalent neurodegenerative disorders worldwide. It is a lifelong and progressive disease that mainly causes complicated cardinal motor symptoms. More than $1 \%$ of the population over 65 years of age is affected, and its prevalence is increased in individuals by aging (Bekris, Mata, \& Zabetian, 2010).

\subsubsection{Clinical Aspects}

In 1817, the clinical features of PD were first depicted in the monograph of "An Essay on the Shaking Palsy" published by James Parkinson. He described the presence of primary motor signs including resting tremor, rigidity, and bradykinesia as characteristics of PD, and the way that the disease progresses over time. Therefore, this neurological disease was named as Parkinson's disease in honor of his contribution (Lees, 2007). Those typical features affect PD patients' voluntary motor movements. Tremor (resting tremor) is the most apparent and notable PD symptom. It can be easily noticed on PD patient's shaking hand when they are at rest. Another characteristic feature of PD, bradykinesia (slowed movement), leads PD patients to make simple tasks difficult and time-consuming due to reduction of speed and amplitude of movements. Muscle rigidity limited PD patients' the range of motion and cause body pain (Jankovic, 2008). However, non-motor symptoms can develop during PD, as well. Cognitive impairment, sleep disturbance, depression, and abnormal behavior, such neuropsychiatric symptoms are common to observe in PD patients. They might be caused by the disease itself or accompanied by disability and inconvenience of daily life. By all means, the patient with PD would be affected by motor dysfunction, but also reduced quality of life.

\subsubsection{PD Pathology}

According to post-mortem studies of PD patients, a lot of cell death of dopaminergic (DA) neurons was observed in the Substantia Nigra pars compacta ( $\mathrm{SNpc}$ ), a region of the midbrain (Figure 1.1). Approximately $60 \%$ of the SN DA neurons were already lost at the onset of PD symptoms. Moreover, the axonal projections of the SN dopaminergic neurons to the striatum are reduced, and near $80 \%$ DA transmitter is depleted. Therefore, it is believed that the loss of SN DA neurons results in the loss of dopaminergic transmission in the 
striatum, leading to the majority of classic motor symptoms of PD. In the past decades, the major emphasis of PD research has been focused to the degeneration of midbrain DA neurons that have been consistently identified as the most severely damaged circuit. However, what causes massive SN DA neuronal death is still not fully understood.

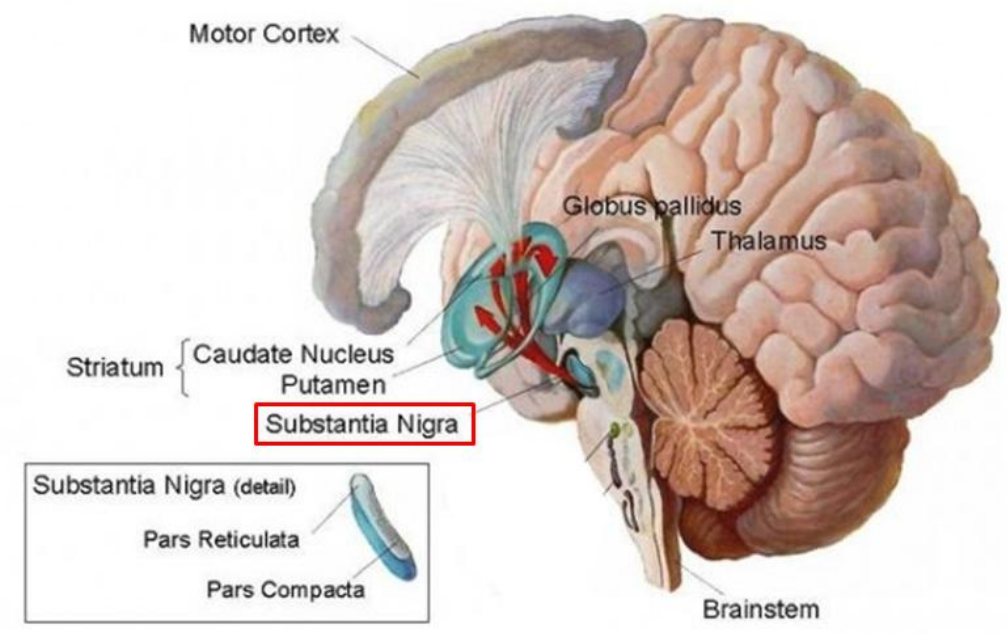

Figure 1.1: Brain Regions Affected by Parkinosn's disease.

The major aspect of PD pathological anatomy is the disappearance of pigmented dopaminergic neurons in the ubstantia nigra (pars compacta), resulting in loss of neuronal projection into the putamen. The image was taken from http://www.doctortipster.com/.

Another PD pathological hallmark is Lewy Bodies (LB). (Figure 1.2) LBs are intraneuronal cytoplasmic inclusions, frequently found in PD patients' brain tissues at autopsy. LBs contain a variety of proteins, neurofilaments, ubiquitin and $\alpha$-synuclein. Among these components, misfolded $\alpha$-synuclein is the predominant constituent of LBs, suggesting that it play a central role in the pathogenesis of PD (Spillantini et al., 1997). Heiko Braak and his colleagues proposed a theory (Braak's theory) for the progression of PD based on LB pathology progression (Braak \& Braak, 2000). However, recent studies have shown that 7-8 \% of PD patients did not present medullary LBs (Kalaitzakis, Graeber, Gentleman, \& Pearce, 2008). Therefore; the cause of PD seems to be more related to the degree of dopaminergic cell loss than to the severity of Lewy pathology. 
Normal aging induces neurodegeneration of DA neurons of SN at a rate of approximately $5 \%$ per decade, in PD patients the neurodegeneration rate is about 10 fold faster (Fearnley \& Lees, 1991). The severity and progression of PD might tightly link to aging induced neurodegeneration.

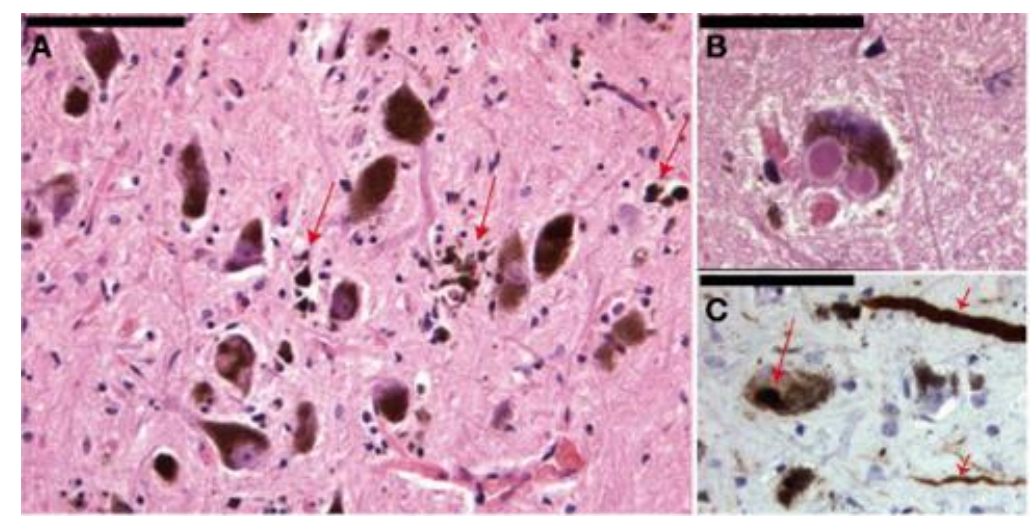

Figure 1.2: Micrographs of mesencephalon Parkinson's disease.

(A) Shows neuronal degeneration in the substantia nigra (arrows) by HE staining. (B) Shows a neuron with two classical Lewy bodies. (C) Shows $\alpha$-synuclein staining with positive staining of a Lewy body (long arrow) and Lewy neurites (short arrows). The image was modified from (den Dunnen, 2013)

\subsubsection{PD Etiology and Environmental Contribution}

Parkinson's disease is a multifactorial neurological disease. Most cases of PD are sporadic: idiopathic disorder may arise from the combinations of environmental and genetic vulnerabilities. The rest of PD ( $5 \%)$ is the result of genetic defects. Although the familial PDs, genetic inheritance, are rare, they share the pathological, biochemical, and clinical features with idiopathic PD. Therefore, researchers have tried to explore the possible mechanisms of PD underlying the Mendelian PD mutations in the past years. A number of genes associated with PD have been identified, either autosomal dominant ( $\alpha$-synuclein, LRRK2, UCHL1) or autosomal recessive (Parkin, DJ-1, PINK1, ATP13A2) (Thomas \& Beal, 2007) (Bekris, et al., 2010). Most of the loci are involved in the impairment of ubiquitin-proteasome system or mitochondrial dysfunctions. Studying those genetic defects provides an insight into PD pathogenesis. Besides, they provide some supportive causes for idiopathic PD (Corti, Lesage, \& Brice, 2011). 
Environmental factors have been suspected to be the critical cause of PD since 1918's influenza pandemic. Infectious agents in the environment affected many individuals leading to the development of Post-encephalitic Parkinsonism (Poskanzer \& Schwab, 1963). The environmental hypothesis was further reinforced by $\mathrm{N}$-methyl-4-phenyl-1, 2, 3, 6-tetrahydropyridine (MPTP) as drug abuse in 1980s (Langston, Ballard, Tetrud, \& Irwin, 1983). Rural pesticides, such as rotenone or paraqua (Ossowska et al., 2006), also have similar adverse effects leading to DA neuron degeneration (Freire \& Koifman, 2012). All of these toxins were subsequently identified to inhibit the activity of mitochondrial complex I, enhance the intracellular reactive oxygen species (ROS), and result in cell death eventually. To this point, by applying these toxins, such as MPTP, animal models can be established for studying PD in vivo (Jackson-Lewis \& Przedborski, 2007).

Investigation the monogenetic defects might shed light on the cellular mechanisms of PD. Nevertheless, the interplay of aging and environmental risks is more likely to contribute to the development of Parkinson's disease.

\subsubsection{PD Causative Hypothesis}

Loss of midbrain dopaminergic neurons and the formation of Lewy Bodies (LBs) are typical characteristics of PD. However, mechanisms underlying this neurodegeneration are not very clear yet. Different hypotheses have been proposed to contribute to neuronal cell death of PD: (1) abnormalities in mitochondrial function and (2) increased level of oxidative stress, (3) defects in protein metabolism through ubiquitin proteasome system (UPS), and (4) autophagy (Schapira \& Jenner, 2011). Besides, the assumption of multifactorial genesis was linked to the interaction of environmental susceptibility and genetic vulnerability. Therefore, the hypotheses mentioned above might be interconnected to each other, resulting in PD pathogenesis.

\subsubsection{Mitochondrial Dysfunction and Oxidative Stress in PD}

Neurons are ATP demanding cells because of the need of energy for impulse transmission. For this reason, mitochondria are enriched in the neuronal cells. Intracellular ATP production is via oxidative phosphorylation and electron transport by five multisubunit complexes (complex I-V) in the mitochondria. During ATP production high amounts of reactive oxygen 
species (ROS) (e.g. superoxide anion $\left(\mathrm{O}_{2}{ }^{-}\right)$, hydrogen peroxide $\left(\mathrm{H}_{2} \mathrm{O}_{2}\right)$ and hydroxyl radicals $(\bullet \mathrm{OH})$ ) can be produced. However, if ROS is generated within a physiological level, those superoxides can be eventually converted to $\mathrm{H}_{2} \mathrm{O}$ by the intracellular antioxidant enzymes (ex. superoxide dismutase, catalase, glutathione peroxidase and glutathione S-transferase, etc.). If the electron transport is blocked (e.g. inhibition of complex I) or mitochondrial function is abnormal (ex. inability of cytochrome c or antioxidant enzyme), it would accumulate excessive ROS and cause serious destruction to the cellular functions, leading to cell death (Fukui \& Moraes, 2008) (Ciccone, Maiani, Bellusci, Diederich, \& Gonfloni, 2013). Therefore, mitochondrial dysfunction and excessive ROS production are common seen in neurodegenerative disorders.

Abundant evidence from PD post-mortem studies supported that oxidative stress induced cell degeneration in the Substantia Nigra. Increased amounts of oxidative damaged lipids, proteins, and DNA were found in sporadic PD patients' affected tissues. ROS detoxification systems were altered, like decreased amount of glutathione $(G S H)$, reduced activity of mitochondrial complex I (Jenner, 2003). In particular, DA neurons are more likely susceptible to oxidative stress due to highly oxidative intracellular environment (Lotharius \& Brundin, 2002). The turnover of the neurotransmitter dopamine (DA) within the cell is processed by monoamine oxidase (MAO), catechol-O-methyltransferase (COMT) or autooxidation. (Figure 1.3) The process of oxidation of DA would result in the production of $\mathrm{H}_{2} \mathrm{O}_{2}$. PD patients have a reduced $\mathrm{H}_{2} \mathrm{O}_{2}$ clearance capacity, suggesting that the incidence of oxidative stress and lesion of antioxidant system might be the cause of PD pathogenesis. 


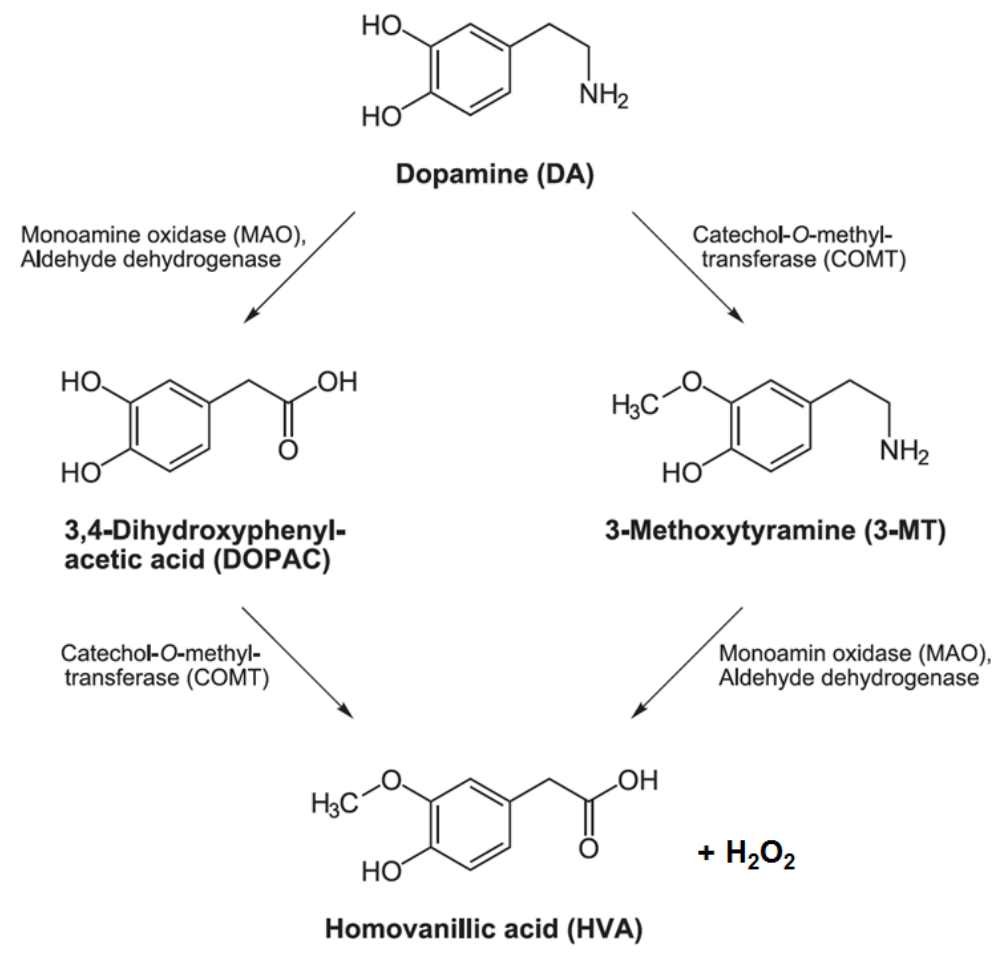

Figure 1.3: Dopamine Degradation.

Dopamine is converted to inactive metabolites by MAO and COMT, accompanied by the production of $\mathrm{H}_{2} \mathrm{O}_{2} \cdot \mathrm{H}_{2} \mathrm{O}_{2}$ is subsequently broken down to free radicals $\left(\bullet \mathrm{OH} \& \mathrm{OH}^{-}\right)$. The cellular antioxidant system would eliminate free radicals and turn into $\mathrm{H}_{2} \mathrm{O}$ eventually. The image was modified from http://en.wikipedia.org/wiki/Dopamine

\subsubsection{Impairment of ubiquitin proteasome system in PD}

The other pathological characteristic of PD is Lewy Bodies (LBs). LB formation is ascribed to the abuse of ubiquitin-mediated protein degradation, resulting in aberrant or misfolded protein aggregations and intracellular presence of proteinaceous deposits. Parkin acts as an E3 ubiquitin ligase, primarily involved in the targeting of aggregation-prone substrates for degradation by the UPS. Genetic evidence showed that defective parkin in PD affected tissues would hamper the protein metabolism, protein aggregations accumulated (Shimura et al., 2000). Especially $\alpha$-synuclein, the major component of LBs, was often found overexpressed or mutant form in either sporadic PD or familiar PD. An impairment of UPS function is one of contributive factors to the proteolytic stress due to fibrilization and aggregation of excess or mutant $\alpha$-synuclein in the cytosol (Bennett et al., 1999) (Tofaris, Layfield, \& Spillantini, 2001). 
As a matter of fact, these cellular pathways might be interconnected. For example, $\alpha$-synuclein is not only involved in the LB formation. The $\mathrm{N}$-terminal membrane-binding domain of $\alpha$-synuclein specifically binds to the mitochondrial membrane and causes mitochondrial fragmentation. The amount of $\alpha$-synuclein localized into mitochondria of SN neurons increases dramatically in PD (Nakamura et al., 2011). PINK1 plays a role of mitochondrial quality control. When the mitochondria is damage, interrupting membrane potential, PINK1 would recruit cytoplasmic parkin to the mitochondria and initiate the autophagic degradation of damaged mitochondria. Both parkin and PINK1 exert functions of ubiquitination and mitochondrial integrity, and implicate these cellular interactions in the PD disease progression (Matsuda et al., 2010). Besides, oxidative damage is also known to disturb ubiquitination and proteasome activity (Jenner, 2007). Thus, combination of oxidative stress, mitochondrial dysfunction, and impairment of UPS are likely to contribute to multifactorial vulnerability of DA neurons degeneration in PD.

\subsubsection{Current Therapeutic Approaches of PD}

Till now there is no cure for Parkinson's disease, but a few of medical treatments could alleviate PD symptoms. 1-3, 4-dihydroxyphenylalanine (L-DOPA), a precursor of dopamine, is a typical first-line drug administration for PD patients. Initially it provides an excellent effect for reducing the motor symptoms. As the progression of the disease, PD patients would experience severe side effects, such as motor fluctuations and dyskinesias (Lang \& Lozano, 1998). Moreover, there are alternative therapeutic options including dopamine agonists, anticholinergic agents, and monoamine oxidase-B (MAO-B) inhibitors (Samii, Nutt, \& Ransom, 2004). Besides, the surgery of deep brain stimulation (DBS) can benefit some population of severe PD patients. By placing the brain pacemaker in PD patient's body and sending electrical impulses to specific parts of the brain, it can help manage some of the PD symptoms and subsequently improve the patient's quality of life (Kleiner-Fisman et al., 2006). In 2006, DBS has been approved for PD treatments by the Food and Drug Administration in United States. Nevertheless, it still carries the risk factors based on the invasion therapy. Some small molecules, ex GDNF or Neurturin, have been proposed to delay or arrest neurodegeneration in PD, but their potential of neuroprotection has not been conclusively approved yet (Obeso et al., 2010). 
Transplantation of fetal mesencephalic tissue in PD patients has been applied in clinical uses. The grafted dopaminergic neurons can restore regulated dopamine release, and provide significant symptomatic relief. In the most successful cases, patients were able to resume an independent life after transplantation. However, the limited source of fetal tissues, occurrence of adverse effects: dystonia, immune rejection, and variability of functional outcome restrict its applications (Lindvall \& Bjorklund, 2004).

Advanced development in stem cell research has provided a new therapeutic approach for Parkinson's disease. Stem cells have the capability of self-renewal and plasticity to generate multilineage cells. Thus, they are a suitable source of various kinds of cells needed for cell therapy. To date, generating dopaminergic neurons from different stem cells have been succeeded. Those include mesenchymal stem cells (MSCs), human embryonic stem cells (hESCs), and induced pluripotent stem cells. However, the cell identity, purity, and quality control of differentiated DA neurons for transplantation should be considered crucially (Pawitan, 2011). In whatever manner, stem cell approaches still hold enormous competence either in cell therapy or modeling diseases.

\subsubsection{Research Models of PD}

Parkinson's disease (PD) is a neurodegenerative disease characterized by the cell death of midbrain Dopamine (DA) neurons and the ensuing locomotor symptoms. Over the past two decades, identification of genetic defects has shed insights into the molecular pathogenesis of PD (Klein \& Schlossmacher, 2007). However, the greatest obstacle for PD research is inaccessible to live PD patients' brain, and lack of patient-specific midbrain DA neurons for mechanistic research and drug discovery. Therefore, in an attempt to mimic the PD neurodegenerative and motor phenotypes different animal models have been created. Administration of neurotoxins (MPTP or 6OHDA) to the striatum area of rats' brain would cause selective dopaminergic neuron death and produce motor deficits. They lead to acute cell death but not fulfill the slow progression of neurodegeneration observed in PD patients. In the other way, PD related genetic studies have been applied to animals, either transgenic or knockout models, even to non-vertebrate systems (e.g. Drosophila, Zebrafish) (Blandini \& Armentero, 2012). Despite the animal models show significant motor phenotype and are useful for unraveling the genetic roles on cellular pathways and mechanism of midbrain DA 
neuron degeneration, there are still some drawbacks, like short life span, less complex brain structure and different physiological states. Furthermore, they could not interpret precisely the authentic features of PD disease in human. A model system that directly reflects the nature of genetics and physiology of the human condition is still urgently demanded ( $\mathrm{Pu}$, Jiang, Zhang, \& Feng, 2012). Thanks to the advance of stem cell biology, pluripotent stem cell seems to overcome the intractable problem and transfers PD research in many prospects.

\subsection{Pluripotent Stem Cells}

Human pluripotent stem cell (hPSC) has the capability of giving rise to almost all of the cell types of body. Therefore, they hold great promise for both scientific research and medical use. To date, a few of methods were established for the generation of hPSC: human embryonic stem cell (hESC) from the inner cell mass of preimplantation embryos, conversion of somatic cells into human induced pluripotent stem cell (hiPSC), and cloned human embryonic stem cell.

\subsubsection{Human Embryonic Stem Cell}

The first human embryonic stem cell line (hESC) was derived from the inner cell masses of blastocysts in 1998 by James Thomson's group (Thomson et al., 1998). They reported that the derived hESC cell lines with normal karyotypes expressed high levels of telomerase activity and presented ES cell specific surface markers. Besides, those hESC cell lines were able to in vivo differentiate to all three germ layers (ectoderm, mesoderm, and endoderm), showing the capacity of pluripotency.

Similar to mouse ES cells, Oct4, Sox2 and Nanog play critical roles in human ES cells. The functions of those master regulators: Oct4, Sox2, and Nanog were subsequently identified to control self-renewal and pluripotency and maintain hESC in the undifferentiated state (Nichols et al., 1998) (Avilion et al., 2003) (I. Chambers et al., 2003) (Hart, Hartley, Ibrahim, \& Robb, 2004) (Boyer et al., 2005). Moreover, unraveling the mechanisms of the lineage commitments allowed hESC to directly differentiate to different types of cells in a dish either for developmental research or clinical purposes (Figure 1.4) (Han, Williams, \& Eggan, 2011) (Kadzik \& Morrisey, 2012) (Spence et al., 2011) (Murry \& Keller, 2008). 


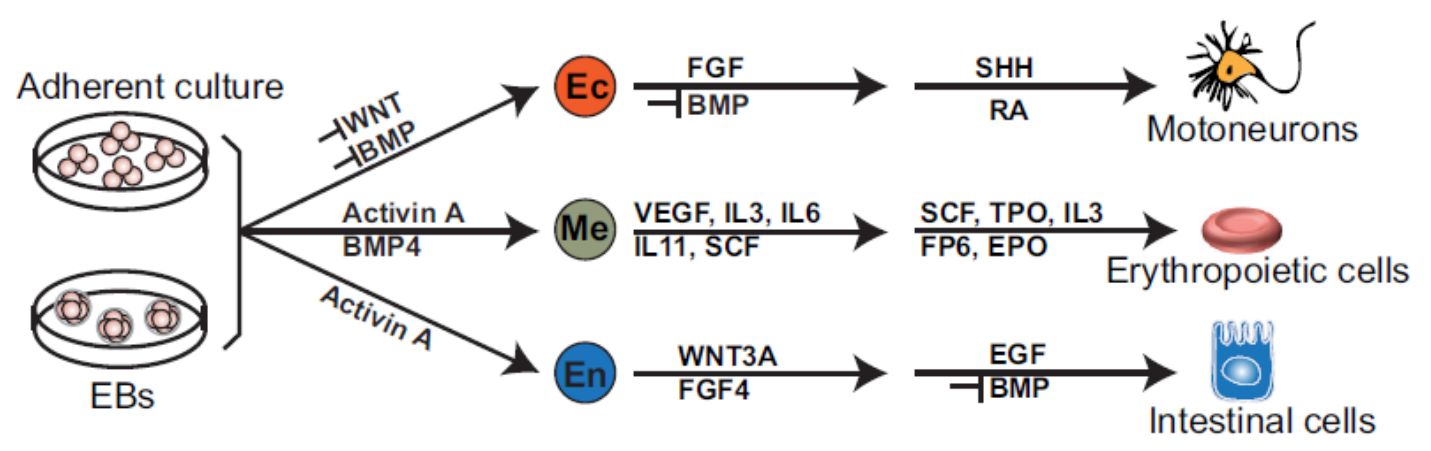

Figure 1.4: Direct Differentiation of hPSCs.

in vitro differentiation of hPSCs can be carried out either by adherent culture or Embryonic bodies (EBs) culture. The presence of growth factors and small molecules activate or inhibit various signaling pathways in a stepwise manner by mimicking embryonic development. Herein three specific examples are illustrated: motorneurons from the ectoderm (Ec), erythropoietic cells from the mesoderm (Me) and intestinal cells from the endoderm (En). The image was taken from (Zhu \& Huangfu, 2013)

hESCs provide power tools for studying human embryogenesis (confirming and distinguishing the finding from mouse genetics), and the potential for clinical use (drug test or differentiated cell for cell replacements) In spite of the fact that isolation of hESCS brought the great expectations, a few of issues should be concerned. One is ethical debate that is about the destruction of human preimplantation embryos for the purpose of generating hESCs. The other one is transplanted non-autologous hESCs might cause immune rejection.

\subsubsection{Human Induced Pluripotent Stem Cell}

Following the breakthrough of 2006's generation of mouse induced pluripotent stem cell (Takahashi \& Yamanaka, 2006), reprogramming human somatic cells to pluripotent state was achieved (Takahashi et al., 2007) (Yu et al., 2007) (Park, Zhao, et al., 2008). By introducing four defined transcription factors: Oct4, Sox2, KIf4, and c-Myc, human somatic cells are allowed to convert to human ES-like cells. These human ES-like cells were similar to ES cells in morphology and cellular characteristics. When transplanted into immunodeficient mice, they grew teratomas including a variety of tissues from three germ cell lineages, showing 
their pluripotency. Thus, they were termed "human induced pluripotent stem cells" (hiPSCs). Those defined factors were also called "Yamanaka factors" for generation of iPSCs. Despite other combinations, including Nanog (Yu, et al., 2007) or hTert (Park, Zhao, et al., 2008), could generate iPSCs, most researchers adopted Yamanaka factors.

To date, iPS technology develops vigorously and attracts extensive attention. Not only that it circumvent the ethical issues concerned in hESCs, the generation of individual hiPSC could also provide the possibility of autologous transplantation, avoiding the risk of immune rejection. Besides, these specific hiPSC cell lines could be used for drug discovery, disease modeling, and the development of cellular transplantation therapies (Bellin, Marchetto, Gage, \& Mummery, 2012). (Figure 1.5)

With the advance of iPS technology, various methods of hiPSC generation developed as well. The initial strategies were retro- or lenti-viral delivery approaches, trying to reach the maximum amount of transduced cells expressing reprogramming factors (Takahashi, et al., 2007) (Yu, et al., 2007). Regarding the transgenic integration and genomic instability, nonviral and nonintegrating methods have been explored. Besides, different sources (ex. skin biopsies, urine (Zhou et al., 2012) or amniotic fluid (Li et al., 2009)) or cell types (ex. fibroblasts, keratinocytes (Aasen et al., 2008) or dental pulp cells (Tamaoki et al., 2010)) have been applied. Table 1.1 lists some distinct and reproducible methods for hiPSC generation. In general, the viral methods are mostly reproducible and highly efficient.

Although iPS technology holds great promise for future medicine, it still keeps some drawbacks. If the transgenic vectors integrate to the host genome, hiPSC might be risky for exogenous reactivation and harmful for further applications, like differentiation or transplantation. Despite hiPSC resembles hESCs in many respects, hiPSC might retain epigenetic memory from the origin of somatic cells, making it more difficult to differentiate to specific cell types. Depending on different approaches of hiPSC generation, cell line-to-cell line differences make it challenging to compare the outcomes either from research or translational medicine (Bellin, et al., 2012). Therefore, iPS technology has been focused on elaborating more efficient \& reproducible nonintegrating delivery methods, establishing standards for cellular characterizations and safety in use. Even if some advanced techniques were established, allowing somatic cells to convert directly to specific cell types (Caiazzo et al., 2011) (Kurian et al., 2013). hiPSC possess the flexibility and unlimited cell source for various cell lineage differentiations, pre-clinical and clinical applications. 


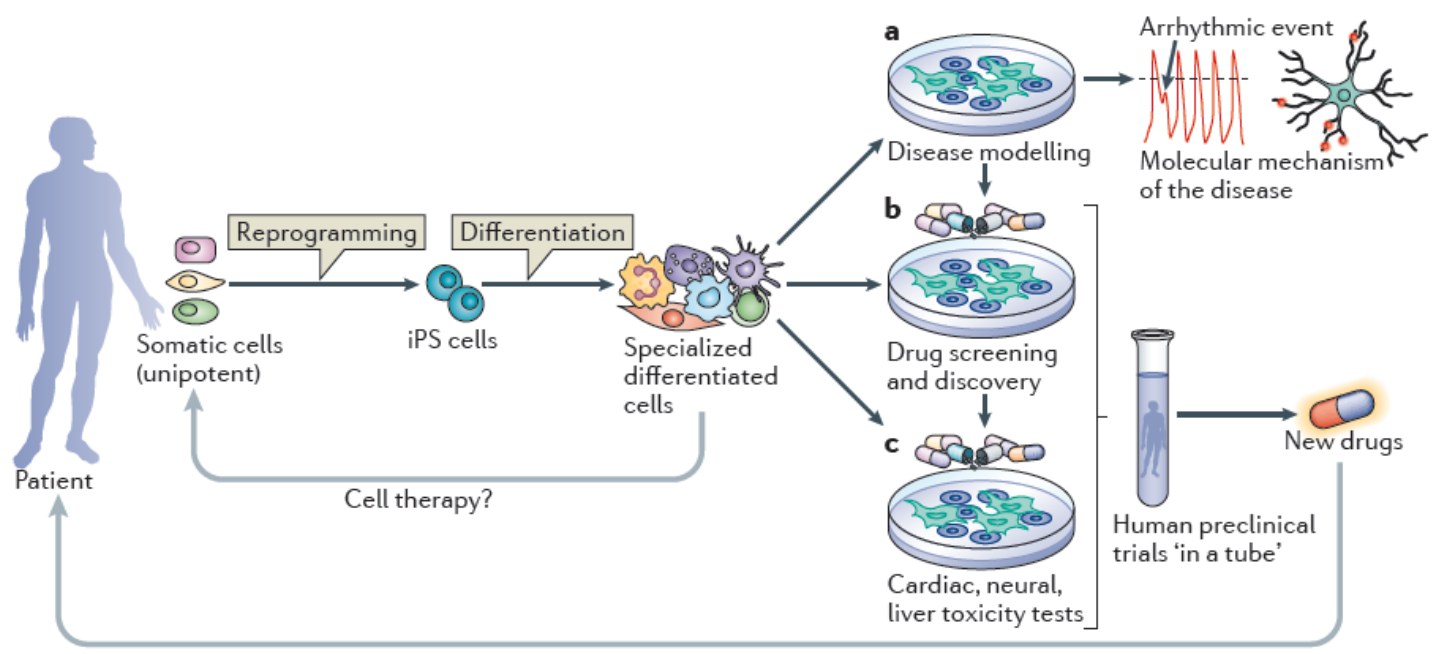

Figure 1.5: hiPSC Derivation, Differentiation, and Application.

Somatic cells from Individuals can be converted to hiPSCs. After direct differentiation, hiPSC derived specialized cells could be used for several applications: (a) disease modeling underlying disease phenotypes, (b) drug screening and discovery, and (c) cellular toxicity tests. hiPSCs provide the potential of autologous cell therapy. The image was taken from (Bellin, et al., 2012)

\subsubsection{Cloned Human Embryonic Stem Cell}

Since the initial discovery in Xenopus (Gurdon, 1962), somatic cell nuclear transfer (SCNT) enable to transplant a nucleus from a mature cell to an enucleated egg and give rise to a living organism. The first cloned mammal, Dolly the sheep and later others (e.g. monkeys) have been created using SCNT (Campbell, McWhir, Ritchie, \& Wilmut, 1996) (Byrne et al., 2007). However, it failed to produce human cloned ES cell in last years. After a long wait, Mitalipov and colleagues have succeeded for the first time in deriving human ES cells by nuclear transfer of human somatic cells to human oocytes (Tachibana et al., 2013). They optimized the SCNT conditions used in monkeys, added extra factors (caffeine) to prevent the premature oocyte activation, and then significantly increased development of human SCNT embryos, cloned human ESC obtained. Cloned hESC displayed normal genome constitutions and received nuclear genome exclusively from parental somatic cells. Gene expression and differentiation profiles in cloned hESC were similar to embryo-derived ESCS. This breakthrough approach will give much hope and another opportunity to prepare 
reprogrammed pluripotent cells for therapeutic cloning and autologous cell transplant. However, the oocytes for use are not easy to acquire legally. The technique for SCNT is not simple to achieve universally. Besides, it is imperative to compare the quality of cloned ES cells and iPS cells in many aspects. 
Table 1.1: hiPSC reprogramming methods

\begin{tabular}{|c|c|c|c|c|c|c|c|c|c|c|c|c|c|c|c|}
\hline 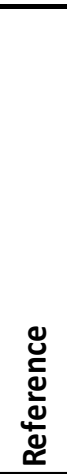 & & 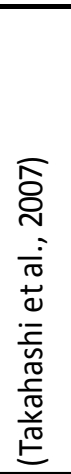 & 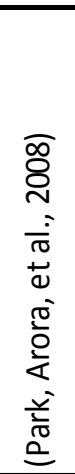 & 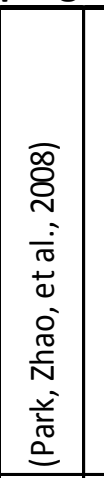 & 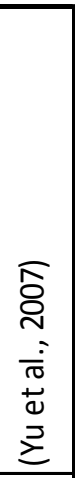 & 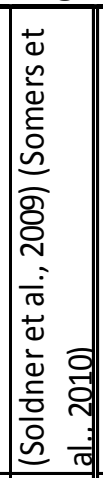 & & 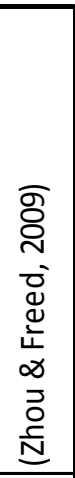 & 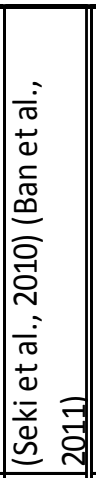 & & 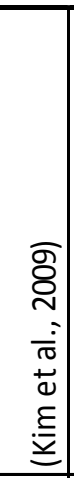 & 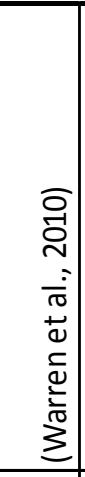 & 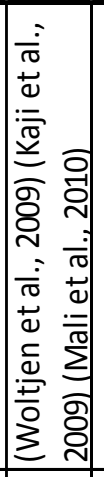 & 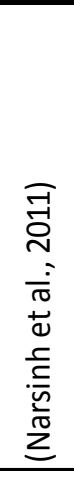 & 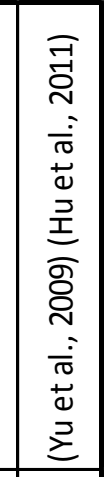 \\
\hline 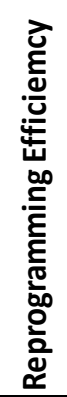 & & $\begin{array}{l}\text { ठे } \\
\text { రे } \\
\text { i }\end{array}$ & 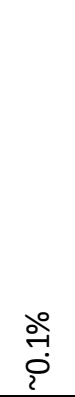 & 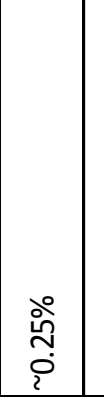 & 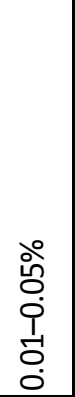 & $\begin{array}{l}\text { ㅇํ } \\
\text { 官 } \\
\text { ¿ें }\end{array}$ & & 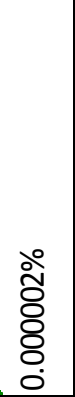 & 웜 & & $\begin{array}{l}\circ \\
\grave{8} \\
\dot{0} \\
\end{array}$ & $\underset{i}{\stackrel{\circ}{i}}$ & 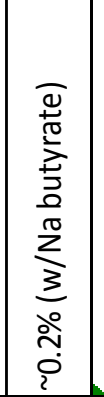 & ㅇํㅇ & \begin{tabular}{|r} 
\\
\\
\\
\end{tabular} \\
\hline 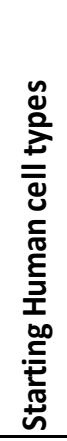 & & 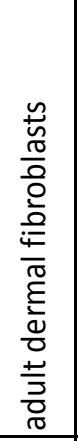 & 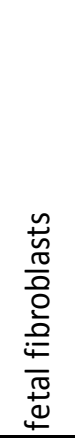 & 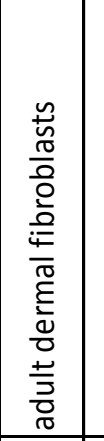 & 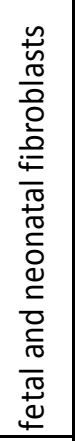 & 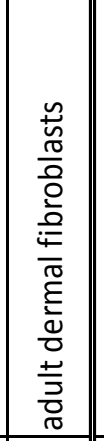 & & 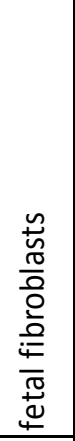 & 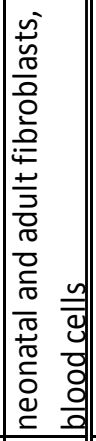 & & 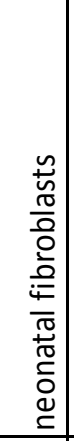 & 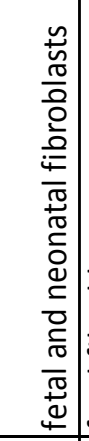 & 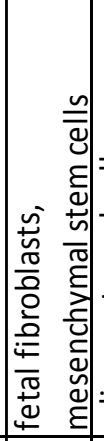 & 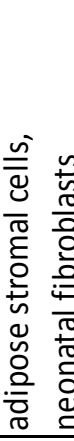 & 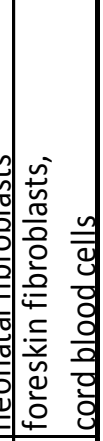 \\
\hline 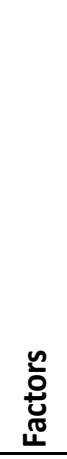 & & 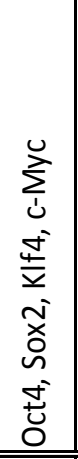 & & 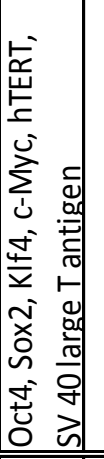 & 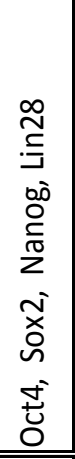 & 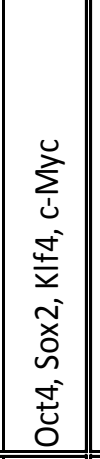 & & 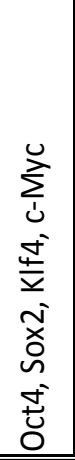 & 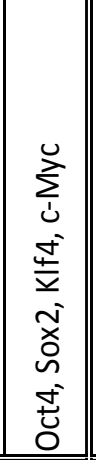 & & 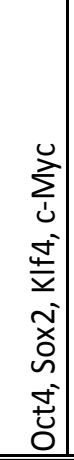 & 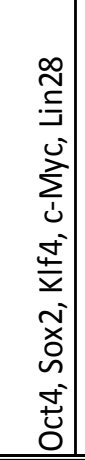 & 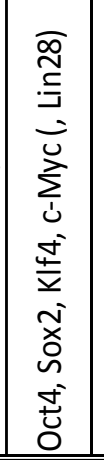 & 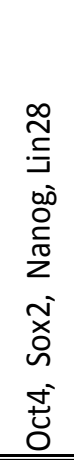 & 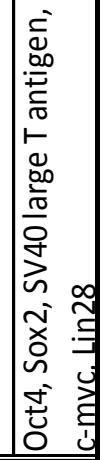 \\
\hline $\begin{array}{l}\frac{n}{0} \\
0 \\
\pm \\
\underline{0}\end{array}$ & 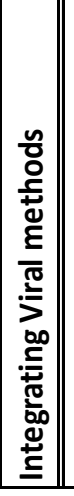 & 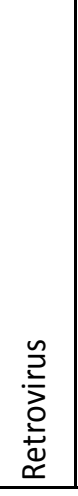 & & & 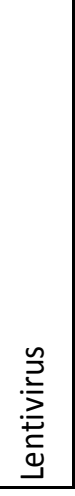 & 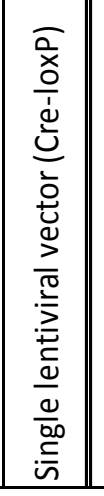 & 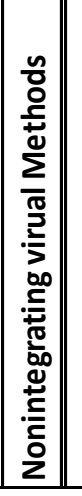 & $\begin{array}{l}\frac{n}{2} \\
\frac{2}{2} \\
\frac{0}{c} \\
\frac{d}{2} \\
\frac{0}{2}\end{array}$ & 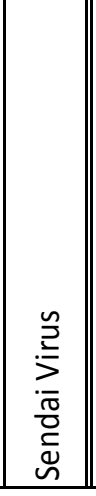 & 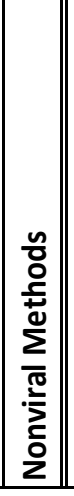 & 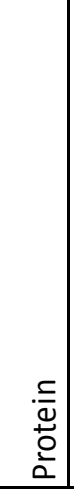 & 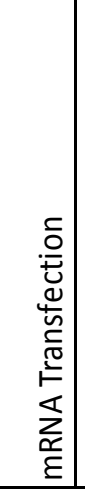 & 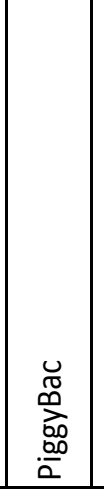 & 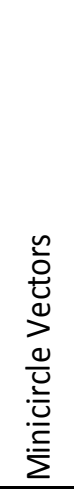 & 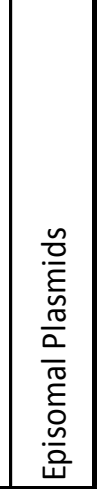 \\
\hline
\end{tabular}




\subsection{Derivation of Dopaminergic Neurons from Human Pluripotent Stem Cell}

Since human ESC was first isolated in 1998, the applications of human pluripotent stem cells (hPSCs) have proposed an extensive scope of possibilities for future regenerative medicine. hPSCs offer a nearly unlimited cell source and are capable of generating therapeutically relevant cell types. Especially, patient specific iPSCs make it possible to model human disease and autologous cell transplantation. In particular, the derivation of DA neurons from hPSCs has drawn an attractive attention either for increasing the knowledge of development and specification of mDA neurons or for understanding the pathogenesis of PD.

Midbrain DA (mDA) neurons play central roles in not only motor control but also motivational, rewarded and cognitive conducts. In virtue of remarkable headway of mouse genetics and mouse ESC, directed differentiation of $\mathrm{mDA}$ neurons has been developed in several ways. Then they were successfully applied in human ESC. Neural lineage was induced from PSCs either by suspension culture of embryoid bodies (EBs) (S. H. Lee, Lumelsky, Studer, Auerbach, \& McKay, 2000) (S. C. Zhang, Wernig, Duncan, Brustle, \& Thomson, 2001) or coculture on stromal feeder layers (PA6 or MS5) (Kawasaki et al., 2000) (Vazin, Chen, Lee, Amable, \& Freed, 2008). Moreover, TGF-B/SMAD inhibitors (Noggin, SB431542, LDN193189 or Dorsomorphin) were employed to accelerate and to dedicate hPSCs to neural lineages (S. M. Chambers et al., 2009). Then neural stem cell/ precursors were isolated and expanded independently. Alternatively subsequent cell fate of mDA neurons was specified by patterning factors, Shh \& FGF8 and undergone for maturation. Then differentiated mDA neurons were characterized by expression of specific markers, like Tyrosine Hydroxylase (TH), engrailed-1 (EN1), or PITX3...etc. Furthermore, detection of dopamine release by HPLC (Studer et al., 1996) and specific physiological properties by electrophysiological assays (Kim et al., 2002) were utilized to determine the functional properties of mDA neurons. Even in vivo engraftment of PSC-derived mDA neurons to adult rodent model approved that they were functional integrated to the brain tissues and restored the behavioral deficits.

The various protocols for increasing the high population of specific mDA neurons have been developed. Forced expression of midbrain-specific markers (e.g. Lmx1a or Nurr1) during neural induction was also used for differentiation of mDA neuron (Andersson et al., 2006). However, the studies of successful engraftment and long-term survival of differentiated mDA neurons were generally poor (Ferrari, Sanchez-Pernaute, Lee, Studer, \& Isacson, 2006) (H. 
Kim et al., 2011). Therefore, an alternative strategy was explored using floor plate-based dopamine neuron differentiation. mDA neurons are believed to originate from floor plate during brain development. Thus, early Shh exposure of hPSC during neural induction would commit high induction of floor plate precursor (Fasano, Chambers, Lee, Tomishima, \& Studer, 2010). Then interplay of Shh and canonical Wnt signaling result in the robust derivation of mDA progenitor coexpressing Foxa2/Lmx1a. Maturation and maintenance of mDA Neuron were subsequently promoted under a set of neurotrophic factors (e.g. GDNF, BDNF, Ascorbic Acid, TGF- $\beta 3$, cAMP). Most importantly, these floor plated-based differentiated mDA neurons show great effects on high amounts of survival cells and rescue motor function after a long time of in vivo engraftment (Kriks et al., 2011). The floor plate-based strategy has been adopted by different groups, modified and optimized to increase mDA neuron specification and application (Xi et al., 2012) (Denham et al., 2012) (Kirkeby et al., 2012)

\subsection{Parkinson's Disease and Human Induced Pluripotent Stem Cell}

PD patient specific iPSC can be generated from their skin fibroblasts, and further differentiated to disease relevant cell type: midbrain dopaminergic neurons. Besides, iPSCS hold a renewable, unlimited cell source and flexibility for PD research in a dish. In recent years, different monogenic PD-iPS cell lines have been established, like SNCA triplication, LRRK2 (G2019S), PINK1 \& Parkin mutants. (Table 1.2) They observed PD phenotypes in PD-iPSC derived DA neurons and revealed possible mechanisms resulting in PD. The increased expression level of $\alpha$-synuclein was found not only in SNCA mutant DA neurons, but also in LRRK2 (G2019S) mutant DA neurons. Moreover, it was often observed that the PD-iPSC derived DA neurons were sensitive to oxidative stress and ROS as evaluated from the analysis of responsive gene transcripts (e.g. cellular stress, antioxidant \& apoptotic genes) were also elevated in affected DA neurons. Mitochondrial damage and impaired clearances of mitochondria (mitophagy) \& aggregated protein were also evident in those PD-iPSC derived DA neuron models. The inhibitions of ERK activity or Drp1 were even proposed to improve the viability of DA neurons (Reinhardt et al., 2013) (Su \& Qi, 2013). Although monogenic forms of PD only take up for a small population of PD cases, it is critically important for understanding the degeneration of DA neurons underlying these 
genetic mutants. It might be a common way to cause PD no matter in familial or idiopathic PD.

Nevertheless the majority of PD cases are idiopathic. Complex interactions between environmental and genetic factors are likely to contribute to the main cause of idiopathic PD. Therefore, DA neurons generated from idiopathic PD patient specific iPSC allow us to compare the genetic compositions and disease phenotypes to discover the vulnerable circumstance. Transplantation of DA neurons derived from idiopathic PD-iPSC into the adult rodent striatum showed that transplanted cells survived and behaved functionally to reduce motor deficits (Hargus et al., 2010). The long-term survival cell rate of transplanted DA neurons was more improved with the floor plate-based differentiation protocol (Kriks, et al., 2011). These transplantation studies provide the practicable prototypes and foundations for the future cell therapy of autologous transplantation. 
Table 1.2: Monogenic PD patients derived hiPSC and DA neurons

\begin{tabular}{|c|c|c|c|c|c|c|c|c|}
\hline Gene & Protein & Mutation & Reference & Donors & Starting cell types & $\begin{array}{l}\text { Reprogramming } \\
\text { factors }\end{array}$ & Affected DA Neuron Phenotype & Perspective \\
\hline \multicolumn{9}{|c|}{ Autosomal dominant } \\
\hline \multirow[t]{3}{*}{ LRRK2 } & LRRK2 & G2019S & (Nguyen et al., 2011) & $\begin{array}{l}160 \text { yr f-patient \& } \\
146 \text { yr f-CTL }\end{array}$ & dermal fibroblast & Retro- OSK & $\begin{array}{l}\text { Increased expression of key oxidative } \\
\text { stress-response genes \& } \alpha \text {-synuclein } \\
\text { protein, sensitive to caspase- } 3 \text { activation } \\
\text { and cell death caused by exposure to } \\
\text { stress agents }\end{array}$ & \\
\hline & & $\begin{array}{l}\text { G2019S } \\
\text { (vs idiopathic PD) }\end{array}$ & (Sanchez-Danes et al., 2012) & $\begin{array}{l}4 \text { LRRK2 (G2019S) } \\
\text { patients, } 7 \text { idiopathic } \\
\text { patient \& } 4 \text { CTLs }\end{array}$ & $\begin{array}{l}\text { dermal } \\
\text { keratinocytes \& } \\
\text { dermal fibroblast }\end{array}$ & Retro- OSK & $\begin{array}{l}\text { LRRK2 PD: increased } \alpha \text {-synuclein } \\
\text { accumulation in cytosol. LRRK2/ iPD: } \\
\text { reduced numbers of neurites \& neurite } \\
\text { arborization, accumulation of autophagic } \\
\text { vacuoles over long-time culture }\end{array}$ & \\
\hline & & G2019S & (Reinhardt et al., 2013) & $\begin{array}{l}255 y r, 82 \text { yr f-patients \& } \\
4 \text { CTLS }\end{array}$ & dermal fibroblast & Retro- OSKM & $\begin{array}{l}\text { Reduced neurite outgrowth \& increased } \\
\text { sensitivity to stress, increased levels of } \alpha \text { - } \\
\text { synuclein, increased MAPK ERK } 1 / 2 \text { activity }\end{array}$ & $\begin{array}{l}\text { Inhibition of ERK activity } \\
\text { rescued mDA neurons as } \\
\text { possible targets for new } \\
\text { therapeutics }\end{array}$ \\
\hline \multirow[t]{2}{*}{ SNCA } & $\alpha$-synuclein & SNCA triplication & (Devine et al., 2011) & $\begin{array}{l}1 \mathrm{f} \text {-patient \& } 1 \mathrm{CTL} \\
\text { (first-degree relative) }\end{array}$ & dermal fibroblast & Retro- OSKM & $\begin{array}{l}\text { Evaluated SNCA expression in RNA \& } \\
\text { protein levels, increased } \alpha \text {-synuclein } \\
\text { secreted from cells }\end{array}$ & \\
\hline & & SNCA triplication & (Byers et al., 2011) & $\begin{array}{l}142 y r \text { m-patient \& } \\
1 \text { CTL (46yr unfeccted } \\
\text { sister ) }\end{array}$ & dermal fibroblast & Retro- OSKM & $\begin{array}{l}\text { Accumulation of } \alpha \text {-synuclein, inherent } \\
\text { overexpression of markers of oxidative } \\
\text { stress, sensitivity to peroxide induced } \\
\text { oxidative stress. }\end{array}$ & \\
\hline
\end{tabular}

Note: Donors--f: female, m: male, yr: age, CTL: control. Reprogrammimg factors--Retro: retrovirus delivery method, Lenti: lentivirus delivery method, O: Oct4, S: Sox2, K: KIf4, M: c-Myc, N: Nanog. 
Table 1.2, cont.

\begin{tabular}{|c|c|c|c|c|c|c|c|}
\hline Gene Protein & Mutation & Reference & Donors & Starting cell types & $\begin{array}{l}\text { Reprogramming } \\
\text { factors }\end{array}$ & Affected DA Neuron Phenotype & Perspective \\
\hline \multicolumn{8}{|c|}{ Autosomal recessive } \\
\hline \multirow[t]{2}{*}{ PARK6 PINK1 } & $\begin{array}{l}\text { Q456X (nonsense) \& } \\
\text { V170G (missense) }\end{array}$ & (Seibler et al., 2011) & $\begin{array}{l}153 \text { yr patient \& } 1 \text { CTL } \\
\text { (healthy family } \\
\text { member) }\end{array}$ & dermal fibroblast & Retro- OSKM & $\begin{array}{l}\text { Reduction of } 80-90 \% \text { PINK1 mRNA level, } \\
\text { mitochondrial depolarization showed } \\
\text { impaired recruitment of overexpressed } \\
\text { Parkin to mitochondria, increased }\end{array}$ & \\
\hline & $\begin{array}{l}\text { V170G } \\
\text { ([Seibler, 2011 \#357]) }\end{array}$ & (Rakovic et al., 2013) & 1 patient \& $1 \mathrm{CTL}$ & dermal fibroblast & Retro- OSKM & $\begin{array}{l}\text { Ubiquitination of endogenous Parkin } \\
\text { attenuates mitophagy }\end{array}$ & $\begin{array}{l}\text { Disease relevant cell } \\
\text { types are faithful in } \\
\text { physological condition }\end{array}$ \\
\hline PARK2 Parkin & PARK2 exon deletions & (Imaizumi et al., 2012) & $\begin{array}{l}272 y r f-, 50 y r \text { m-patient } \\
\& 2 \text { CTLs }\end{array}$ & dermal fibroblast & Retro- OSKM & $\begin{array}{l}\text { Increased oxidative stress and enhanced } \\
\text { activity of Nrf2 pathway, exhibited } \\
\text { abnormal mitochondrial morphology \& }\end{array}$ & \\
\hline & PARK2 exon deletions & (Jiang et al., 2012) & 2 patients $\& 2$ CTLS & dermal fibroblast & Lenti- OSKN & $\begin{array}{l}\text { Increased spontaneous DA release, } \\
\text { reduced DA uptake and DAT-binding sites, } \\
\text { elevated ROS by increasing MAO } \\
\text { transcripts }\end{array}$ & $\begin{array}{l}\text { Parkin controls } \\
\text { dopamine utilization in } \\
\text { human midbrain DA } \\
\text { neurons }\end{array}$ \\
\hline
\end{tabular}




\subsection{Aim of this Study}

Parkinson's disease (PD) is a multifactorial neurodegenerative disease. The past decades PD research has drawn numerous attentions, trying to explore the disease mechanism and the medical therapies. A few sets of genetic mutants found in familial PD were identified, and their roles have been linked to mitochondrial dysfunction and impairment of UPS, given the insights of degeneration of mDA neuron, the central cause of PD. Although many efforts have been done in the animal models, the authentic PD patients' affected mDA neuron model is still imminently demanded. Despite the inaccessibility of PD patients' live brain tissue, we are able to generate PD patient specific iPSC from skin fibroblasts and further differentiate to mDA neurons.

In this study, we followed the workflow (Figure 1.6). We attempted to recruit PD patients from different kindred. Together with healthy persons, PD patient specific iPSC would be generated and characterized their states of pluripotency. Neural precursors would be then derived from these control and PD-iPSCs and applied for transcriptome analysis. By gene expression profiles between control and PD patients' neural precursors, differential cellular networks and novel molecular players might be perceived. More importantly, the phenotypes of mDA neurons derived from control and PD-iPSC would be examined using external stress stimulations, like mitochondrial damage and oxidative stress. By establishing the PD patient specific hiPS cell models, we expect to obtain the new perception for understanding Parkinson's disease. 


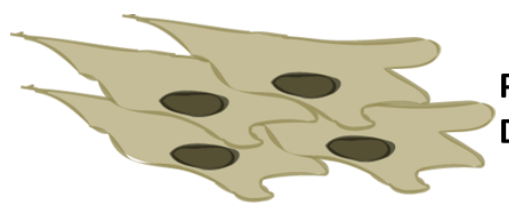

PD patients' / healthy person

Dermal fibroblasts

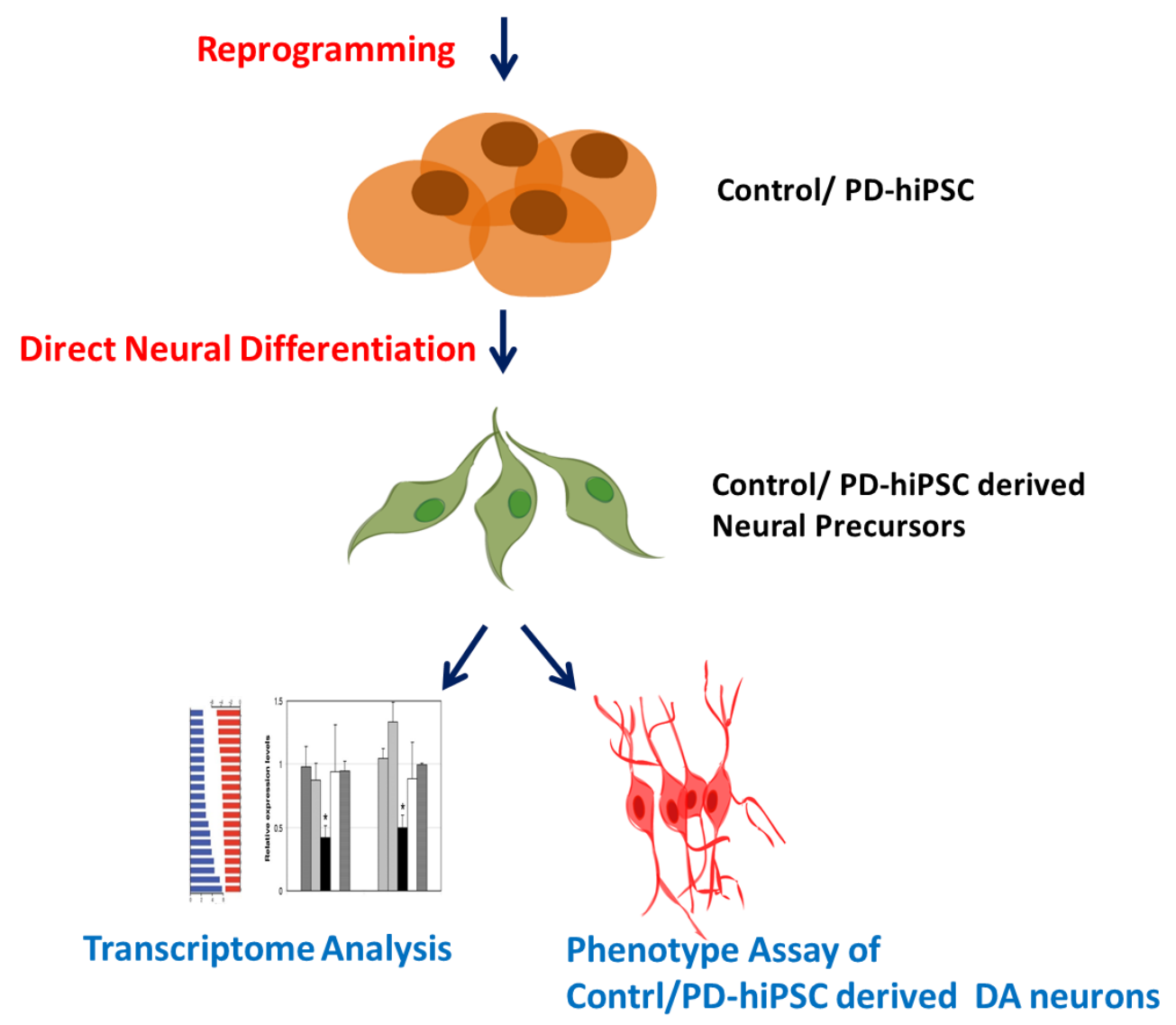

Figure 1.6: Work Flow of the Study 


\section{Material \& Methods}

\subsection{Methods}

\subsection{1 in vitro Cell Cultures}

\subsubsection{Isolation of Mouse Embryonic Fibroblasts (MEFs)}

The Procedure was performed by following the protocol (Mansouri, Fukumitsu, Schindehuette, \& Krieglstein, 2009) which was published from our lab. Briefly, mouse embryos were removed from E13.5 CD1 pregnant mice. Their heads, limbs, tails, and viscera were cut off. The rest of the body was minced into small pieces, and incubated with Trypsin for $30 \mathrm{~min}$ at $37^{\circ} \mathrm{C}$. Then the cell pellet was centrifuged down for $15 \mathrm{~min}$ at $1000 \mathrm{rpm}$, and plated on gelatinized $14.5-\mathrm{cm}$ dishes. The plated MEFs were fed with MEF medium at $37^{\circ} \mathrm{C}$. When newly prepared MEFs reached up to $90 \%$ confluence, the cells were either frozen as PO or subculture for expansions. In general, MEFs can be passaged up to 4 passages for the use of feeder layers.

\section{Mitotic Inactivation of MEFs}

80-90\% confluent MEFs were treated with Mitomycin $\mathrm{C}(10 \mu \mathrm{g} / \mathrm{mL})$ for $2.5 \mathrm{~h}$ at $37^{\circ} \mathrm{C}$, and either directly replated to $0.2 \%$ gelatinized plates as feeder layers or frozen for future uses.

\subsubsection{Culture of Human Pluripotent Stem Cells (PSCs)}

Human pluripotent stem cells (hPSCs), like human embryonic stem cells ( $\mathrm{H} 1$ and $\mathrm{H} 9$ ) and human induced pluripotent stem cells (hiPSCs), were grown and maintained on a layer of mitotically inactivated MEFs (iMEFs). One day before plating PSCs, iMEFs were plated on gelatinized $3.5-\mathrm{cm}$ dishes $\left(20000\right.$ cells $\left./ \mathrm{cm}^{2}\right)$. When hPSCs colonies were grown big enough, hPSCs were passaged by treatments of Collagenase IV $(1 \mathrm{mg} / \mathrm{ml})$ to lift the edges of cell colonies and manual splitting using cell scrapers. The hPSCs colonies were collected in a 15 $\mathrm{ml}$ Falcon tube and pulled down to the bottom by gravity in a few minutes. hPSCs colonies were washed by hPSCs medium, and the suspension containing iMEFs and Collagenase IV was removed. Washing step was repeated twice. Then hPSCs colonies were carefully broken into smaller pieces by pipetting and replated to new iMEF feeder dishes at the ratio of 1:3 1:5. hPSCs were fed with hPSC medium supplemented with bFGF $(10 \mathrm{ng} / \mathrm{ml})$ at $37^{\circ} \mathrm{C}$. The medium was changed daily. hPSCs were passage about every $5^{\sim 7}$ days. Working with 
human ES cells (H1, H9) imported from WiCell (Madison, Wisconsin) was approved by the Robert-Koch Institute in Berlin (AZ. 1710-79-1-4-5) according to the German law.

\subsubsection{Generation of Human Induced Pluripotent Stem cells (hiPSCs)}

For production of lentivirus, $2^{\text {nd }}$ generation Packaging System was applied in this work. To produce virus, a single packaging plasmid (psPAX2), an envelope plasmid (pMD2.G), and the transfer vector (hSTEMCCA-loxP) were included. Prior to transfection, HEK293T/T17 (ATCC, CRL-11268) (293T) were grown to 80-90\% confluence in a gelatinized T75 flask. By the time transfection mixtures were first prepared as following:

(hSTEMCCA: psPAX2: pMD2.G = 10:2:1) $15 \mu \mathrm{g}$ total plasmid/ $500 \mu \mathrm{l}$ DMEM

$45 \mu$ l Lipofetamine 2000/500 $\mu$ I DMEM

, mixed gently and incubated at room temperature ( $\mathrm{rt}$ ) for $20 \mathrm{~min}$ according to the manufacturer's instruction (Lipofetamine 2000, Invitrogen, 11668-019). Meanwhile, 293T cells were fed with fresh $9 \mathrm{ml}$ 293T medium. Then the $1 \mathrm{ml}$ transfection mixture were slowly added to the $293 \mathrm{~T}$ cells and incubated at $37^{\circ} \mathrm{C}, 5 \% \mathrm{CO}_{2}$. Supernatant was carefully collected at $24 \mathrm{~h}$ and $36 \mathrm{~h}$ after transfection, and filtered through a $45 \mu \mathrm{m}$ filter (Sartorius) to get rid of cell debris. The remaining supernatant was centrifuged for $1.5 \mathrm{~h}$ at $48960 \mathrm{~g}$ in an ultracentrifuge. The viral pellets were resuspended in $100 \mu \mathrm{lPBS}$ at $4^{\circ} \mathrm{C}$ overnight. The volume of $10 \mu \mathrm{l}$ for each aliquot was made and stored at $-80^{\circ} \mathrm{C}$.

\section{Lentiviral Infection and Human iPSC Generation}

$1 \times 10^{5}$ fibroblasts derived from patients or controls were infected with concentrated lentivirus in hFB medium supplemented with $5 \mu \mathrm{g} / \mathrm{ml}$ polybrene. The next day, the infectious medium was replaced by the fresh hFB medium. On day 3 , the infected cells were transferred onto iMEF feeder plates. After $24 \mathrm{~h}$, the medium was switched to hPSC medium containing $10 \mathrm{ng} / \mathrm{ml} \mathrm{bFGF}$ and Sodium Butyrate $(0.5 \mathrm{mM})$. The medium was changed every other day. On day 10, the cells were fed with hPSC medium supplemented with $10 \mathrm{ng} / \mathrm{ml}$ bFGF. About 20 days later, the ES-like colonies started to appear. Then hPSC medium was fed every day. One month after infection, the ES-like colonies were about to grow big enough. The ES-like colonies were manually picked at stereomicroscope, transferred onto fresh iMEF feeder plates, and expanded under the standard hPSC culture conditions. hiPSC colonies, 
some were frozen in liquid nitrogen when expanded to passage $4-5$, and some were grown for further application.

\section{Excision of hSTEMCCA}

The excision of hSTEMCCA vector from the hiPSC colonies was based on Cre/loxP recombination by transient overexpression of Cre recombinase in the cells. Delivery of Cre expression vector (pHAGE2-Cre-IRES-PuroR) into the cells was followed the procedure of reverse transfection using Lipofetamine 2000 Transfection Reagent (Invitrogen, 11668-019). One day before transfection, puromycin antibiotic resistant iMEFs (Cell Biolabs, CBA-312) were plated on gelatinized $3.5-\mathrm{cm}$ dishes. hiPSCs were fed with hPSC medium containing 10 $\mu \mathrm{M}$ Y27632, additionally. At the time of transfection, transfection mixtures were first prepared as following:

\section{$2 \mu \mathrm{g}$ Cre plasmid/ $50 \mu \mathrm{l} \mathrm{KO-DMEM}$ \\ $10 \mu$ l Lipofetamine 2000/50 $\mu \mathrm{l}$ KO-DMEM}

, incubated for $5 \mathrm{~min}$ at room temperature $(\mathrm{rt})$. Then those two were mixed by gently pipetting, and incubated for another $30 \mathrm{~min}$ (maximum $50 \mathrm{~min}$ ) at rt. Meanwhile, hiPSC colonies were dissociated into single cells by using Accutase. 800,000 hiPSCs were counted and suspended in $100 \mu \mathrm{l}$ hPSC medium (10 $\mu \mathrm{M}$ Y27632). Transfection mixtures were added into suspended hiPSC gently for additional 15 min incubation, then plated on puromycin resistant iMEFs feeder dishes. The next morning, hPSC medium (10 $\mu \mathrm{M}$ Y27632) was freshly replaced. By the evening, puromycin selection $(1.2 \mu \mathrm{g} / \mathrm{ml})$ was begun $24 \mathrm{~h}$ following the initial transfection and lasted for $48 \mathrm{~h}$. The transfected hiPSC cells were kept on feeding hPSC medium (10 $\mu \mathrm{M}$ Y27632 (ROCK inhibitor)) for one week, then MEF-conditioned hPSC medium (10 ng/ml bFGF) were replaced. The re-emergence of hiPSC colonies was noted within 1-2 week, and 5-7 colonies from each dish were picked on day 11-14 for passaging. PCR of gDNA extracted from each subclone was performed to screen for excision of the hSTEMCCA vector using the STEM_SPM primers (Table 2.3 ), $95^{\circ} \mathrm{C}$ for $3 \mathrm{~m}$; followed by 33 cycles of $94^{\circ} \mathrm{C}$ for $30 \mathrm{sec}, 60^{\circ} \mathrm{C}$ for $30 \mathrm{sec}$, and $72^{\circ} \mathrm{C}$ for $1 \mathrm{~m}$; followed by a single cycle of $72^{\circ} \mathrm{C}$ for $5 \mathrm{~m}$. PCR products were analyzed by running on $2 \%$ agarose gels. Furthermore, vector excision was confirmed by Southern blotting. The vector free hiPSC cells were either frozen in liquid nitrogen or kept on growing for further study. 


\section{MEF-Conditioned hPSC Medium}

About 6.6 million mitotic inactive MEFs were plated on T75 flask in MEF medium and incubated overnight at $37^{\circ} \mathrm{C}, 5 \% \mathrm{CO}_{2}$. The medium was switched to $15 \mathrm{ml} \mathrm{hPSC}$ medium containing $5 \mathrm{ng} / \mathrm{ml} \mathrm{bFGF}$, and kept incubated at $37^{\circ} \mathrm{C}, 5 \% \mathrm{CO}_{2}$. The MEF-Conditioned hPSC medium was collected and replaced by fresh medium onto MEFs daily for 7 days. The collected MEF-Conditioned hPSC medium was filtered through $0.22 \mu \mathrm{M}$ filter and stored at $-80^{\circ} \mathrm{C}$ in aliquots. Before use, additional $10 \mathrm{ng} / \mathrm{ml} \mathrm{bFGF}$ was added freshly into MEF-Conditioned hPSC medium.

\subsubsection{4 in vitro Differentiation}

Embryoid body (EB) formation is one of the easiest procedures for in vitro differentiation of PSCs. PSCs were dissociated into small pieces by using Collagenase IV (1 mg/ml) and manual splitting. The small clumps of cells were washed twice with hPSC medium to remove MEFs, and cultured in suspension. After 4-5 days, embryonic bodies (EBs) were formed. EBs were transferred to gelatinized plates or coverslips to grow for additionally 7 days for further differentiation. During the course of the differentiation, cells were fed with differentiation medium in the absence of bFGF. The differentiated cells were examined by immunocytochemistry for differentiation markers: Tuj1 for Ectoderm layer, AFP for Endoderm layer, and Brachyury for Mesoderm layer.

\subsubsection{5 in vivo Differentiation}

The in vivo differentiation experiments (teratoma formation) were performed by Prof. Dr. Ralf Dressel in the Institute of Human Genetics, University of Göttingen. At least 2 million hiPSC cells were harvested by Accutase and suspended in hPSC medium containing $10 \mu \mathrm{M}$ Y27632 and 30\% matrigel. The cells were injected into SCID or RAG-/- mice. The mice were carefully monitored for the appearance of tumors. If tumors were formed, the mice were sacrificed. Tumors were collected for histological analysis by paraffin embedding and hematoxylin and eosin (HE) staining. 


\subsubsection{Derivation and Maintenance of Human Neural Precursors}

hiPSCs were harvested by $1 \mathrm{mg} / \mathrm{ml}$ Collagenase IV and plated on PA6 stromal feeder dishes (400,000 PA6 cells/3.5-cm dish). hiPSCs were fed with Neural Induction medium which contained BMP inhibitors (noggin $200 \mathrm{ng} / \mathrm{ml}$ or Dorsomorphin $2 \mu \mathrm{M}$ ) every second day. After 1 2 weeks, neural rosette structures started to appear with hiPSC colonies. By manually dissecting under the stereomicroscope, neural rosettes were transferred to poly-D-Lysine (PDL)-coated plates, and fed with NP medium supplemented with bFGF $20 \mathrm{ng} / \mathrm{ml}$ and EGF 20 $\mathrm{ng} / \mathrm{ml}$.

\section{Maintenance of Neural Precursor Cells}

After some days of incubation, NPs outgrew from neural rosette structure. Following Accutase treatments, the cells became round in shape, then the cells were collected, centrifuged, suspended in NP medium, and plated to new plates. Then neural precursor cells (NPs) were grown as monolayers on NP medium (bFGF \& EGF) at $37^{\circ} \mathrm{C}$ and $5 \% \mathrm{CO}_{2}$. Media were changed daily or every other day. Cells were passaged every $5 \sim 7$ days. Cells can be frozen with NP medium containing $10 \%$ DMSO in liquid nitrogen. So far, neural precursors can be maintained up to passage 25 .

\section{Neuronal Differentiation}

50-75 $\times 10^{4}$ NPs were seeded on matrigel (BD Bioscience, 356230) coated 3.5-cm culture dishes or glass coverslips. The minimal supportive medium for differentiating neural cells into neurons is neural differentiation medium containing BDNF $10 \mathrm{ng} / \mathrm{mL}$ and $200 \mu \mathrm{M}$ Ascorbic Acid. Differentiations are usually carried out for 1 2 weeks with media changes every two to three days.

To enrich for dopaminergic neurons, the NPs were first fed with neural differentiation medium supplemented with $100 \mathrm{ng} / \mathrm{ml} \mathrm{FGF8} \mathrm{\&} 100 \mathrm{ng} / \mathrm{ml}$ Shh (or $2 \mu \mathrm{M}$ Purmorphamine) for neuron patterning. The medium was changed every other day. After 2 weeks, for neuron maturation, the cells were fed with neural differentiation medium containing $10 \mathrm{ng} / \mathrm{ml}$ GDNF, $10 \mathrm{ng} / \mathrm{ml} \mathrm{BDNF}, 1 \mathrm{ng} / \mathrm{ml}$ TGF- $\beta 3,200 \mu \mathrm{M}$ Ascorbic Acid and $0.5 \mathrm{mM}$ dbcAMP for subsequent weeks. Cells were analyzed after day 30 following the onset of differentiation procedure. 


\section{Culture of PA6 Stromal Cells}

PA6 cells (RIKEN Bio Resource Center Cell Bank), bone-marrow derived stromal cells, were maintained in PA6 medium. Upon passage, PA6 cells were split by the ratio of 1:5. For inactivation, cells were treated with Mitomycin $\mathrm{C}(10 \mu \mathrm{g} / \mathrm{mL})$ for $1 \mathrm{~h}$ at $37^{\circ} \mathrm{C}$, and either directly replated to $0.2 \%$ gelatinized plates as feeder layers or frozen for future uses.

\subsubsection{Differentiation of Floor-Plate Derived Dopaminergic Neurons}

Midbrain dopaminergic neurons are believed to originate from the midbrain floor plate (FP) during the brain development. Herein, the differentiation protocol was modified from (Xi, et al., 2012). hiPSCs were plated on PA6 stromal feeder dishes. The next day, hiPSC were fed with FP induction medium in the presence of $10 \mu \mathrm{M}$ SB431542, $0.1 \mu \mathrm{M}$ LDN193189, $2 \mu \mathrm{M}$ purmorphamine (pur), and $0.5 \mu \mathrm{M}$ CHIR99021 (CHIR). The medium was changed every second day. In addition, Shh (C24II) $100 \mathrm{ng} / \mathrm{ml}$ was added for the first four days. On day 9, SB431542 and LDN193189 were removed. The cells were fed with FP medium (Pur \& CHIR) till day $11 \sim 13$. On day 11 , the cells were replated on Laminin $(5 \mu \mathrm{g} / \mathrm{ml}) /$ Fibronectin $(5 \mu \mathrm{g} / \mathrm{ml})$ or on matrigel coated coverslips for immunostaining. Cells were continuously maintained on

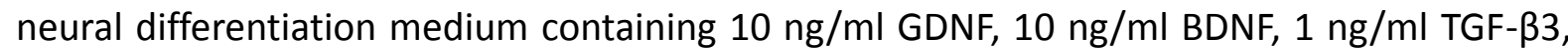
$200 \mu \mathrm{M}$ Ascorbic Acid, $0.5 \mathrm{mM}$ dbcAMP, and DAPT $5 \mu \mathrm{M}$. About day 23 25, the differentiated cells were replated on either coverslips or new dishes for future studies. After one month of differentiation, or even longer (till 40 45 days), the differentiated cells were harvested for molecular and functional analysis.

\subsubsection{Biochemistry \& Molecular Biology Methods}

\subsubsection{Alkaline Phosphatase Assay}

hiPSC colonies were washed with PBS once, and fixed by $3.7 \%$ Formaldehyde for 20 min at room temperature $(\mathrm{rt})$. Followed 3 times of PBS-washes, fixed cells were washed $3 \times 10 \mathrm{~min}$ in NTMT solution to equilibrate to alkaline $\mathrm{pH}(9.0-9.5)$. Then the cells were exposed to NBT/BCIP (Roche, 11681451 001) diluted NTMT (200 $\mu \mathrm{l} / 10 \mathrm{ml}$ NTMT) in the dark with gentle shaking. If color developed, the reaction was stopped by removing NBT/BCIP, and washed by PBS. 


\subsubsection{Fluorescent Immunocytochemistry}

To detect the expression of proteins by immunocytochemical methods, the cells were grown on either matrigel or PDL/laminin/fibronectin coated coverslips. At the time of assays, cells were rinsed with PBS once and fixed by $3.7 \%$ Formaldehyde for $20 \mathrm{~min}$ at rt. The fixed cells were washed with PBS and permeabilized by $0.5 \%$ Triton in PBS for $10 \mathrm{~min}$. The cells were washed again and blocked in the blocking solution (5\% BSA and $0.1 \%$ Triton in PBS) for $1 \mathrm{~h}$ at rt. Primary antibodies were diluted in blocking solutions, and incubated with cells either overnight at $4^{\circ} \mathrm{C}$ or $2 \mathrm{~h}$ at rt. After that, cells were washed 3 times with PBST (0.1\% Tween-20 in PBS), 10 min each. Diluted fluorescent secondary antibodies were applied for conjugation of respective primary antibodies, and they were incubated for $1 \mathrm{~h}$ in the dark. Then the coverslips were washed 3 times with PBST. Nuclear staining was counterstained by DAPI (Vector Labs). The coverslips were sealed by nail polish and stored at $4^{\circ} \mathrm{C}$ in the dark.

The fluorescent images were acquired by using Leica SP5 Confocal microscope and Olympus BX60 fluorescent microscope. The processed images were done by Adobe Photoshop CS2 and arranged by Adobe Illustrator CS3. Used primary and secondary antibodies are listed in Table $2.1 \& 2.2$

\subsubsection{Isolation of total RNA}

Total RNA was isolated with the RNeasy Mini Kit (Qiagen, 74104) according to the manufacturer's instructions. Briefly, collected cells were lysed and RNA was purified using silica-membrane RNeasy spin columns. DNA removal was carried by on-column DNase treatment. The concentration and quality of purified RNA were determined by the NanoDrop (Peqlab).

\subsubsection{4 cDNA synthesis}

0.5-1 $\mu$ g total RNA was subjected to cDNA synthesis with QuantiTect Reverse Transcription Kit (Qiagen, 205311) following the manufacturer's instructions. The RNA template was annealed by a unique RT Primer Mix and transcribed into cDNA by QuantiTect Reverse Transcriptase. The final volume was equal for each sample (e.g. $20 \mu l$ ). The synthesized cDNA was stored at $-20^{\circ} \mathrm{C}$ or optimized for use in conventional PCR or QRT-PCR. 


\subsubsection{Quantitative Real-Time-PCR (QRT-PCR)}

To quantify the relative expression of specific genes was relied on QRT-PCR. Target genes were amplified by their own specific primer set and normalized by housekeeping genes (GAPDH or Actin). For each reaction mix was total $10 \mu \mathrm{l}$ :

Forward / reverse primer mix (5 $\mu \mathrm{M}$ each) $1 \mu \mathrm{l}$

2X SYBR Green PCR Master Mix (KAPA SYBR FAST Universal) $5 \mu l$

$$
\mathrm{ddH}_{2} \mathrm{O} 2 \mu \mathrm{l}
$$

cDNA diluent (1:2-1:5) $2 \mu \mathrm{l}$

The PCR was performed on the Mastercycler realplex system (Eppendorf).

\subsubsection{Isolation of genomic DNA}

Collected cell pellets were lysed in $500 \mu$ l DNA lysis buffer and $50 \mu$ l Proteinase $\mathrm{K}(10 \mathrm{mg} / \mathrm{ml})$ at $56^{\circ} \mathrm{C}$ overnight. The next day, $500 \mu$ I Isopropanol was added to precipitate the genomic DNA. After $10 \mathrm{~min}$ of centrifugation (13.000 rpm), the supernatant was discarded, and $1 \mathrm{ml}$ $70 \%$ ethanol was added to wash the cell pellets. Samples were centrifuged again for $5 \mathrm{~min}$ (13.000 rpm) and the supernatant was discarded. The pellets were air-dried for $5 \mathrm{~min}$ and dissolved in 10-50 $\mu \mathrm{l} \mathrm{TE} \mathrm{buffer,} \mathrm{depending} \mathrm{on} \mathrm{the} \mathrm{concentration} \mathrm{needed.} \mathrm{The} \mathrm{concentration}$ and quality of resulting genomic DNA were measured by using NanoDrop.

\subsubsection{Fingerprinting}

The method was referred by (Park, Arora, et al., 2008). 50 ng of genomic DNA was used to amplify genomic regions containing highly variable numbers of tandem repeats (VNTR) in order to verify the genetic relatedness of iPS cell lines relative to their parent fibroblasts. The sequences of each primer set: D10S1214, D17S1290, D7S796 and D21S2055 were listed in Table 2.4. PCR conditions were: $94^{\circ} \mathrm{C}$ for $10 \mathrm{~min}$ followed by 35 cycles of $94^{\circ} \mathrm{C}$ for $1 \mathrm{~min}$, $55^{\circ} \mathrm{C}$ for $1 \mathrm{~min}$ and $72^{\circ} \mathrm{C}$ for $1 \mathrm{~min}$, with a final extension step of $72^{\circ} \mathrm{C}$ for $5 \mathrm{~min}$. PCR products were analyzed by running on $2.5 \%$ agarose gels.

\subsubsection{Bisulfite sequencing}

The EpiTect Bisulfite Kit (Qiagen, 59104) was used for bisulfite conversion of genomic DNA, unmethylated Cytosine nucleotides (C) to Uracil nucleotides (U). The procedure was 
conducted as the manufacturer's instructions. The promoter region of OCT4 were amplified by the subsequent PCR using primers (Table 2.6) previously described ((Freberg, Dahl, Timoskainen, \& Collas, 2007) primer pair 4 for OCT4). The resulting amplified products were cloned into pTZ57R/T Cloning Vector (Fermentas, K1214), amplified in DH5 $\alpha$ cells. The cloned plasmids were purified by the QIAprep Spin Miniprep Kit (Qiagen, 27106) and sequenced by ABI PRISM ${ }^{\circledR} 3100$ Genetic Analyzer (Applied Biosystems).

\subsubsection{Southern Blot}

After overnight digestion with BamHI, $10 \mu$ g genomic DNA fragments were resolved on $0.8 \%$ agarose gel with low voltage (25 V) over 16 hours. The gel was soaked in $0.25 \mathrm{M} \mathrm{HCl}$ for 15-20 min with gentle shaking. This step is necessary for the optimal transfer of DNA fragments $>4 \mathrm{~kb}$ to the blotting membrane. Two 20 min-denaturation washes followed in denaturing buffer. Then the other two 20 min-neutralization washes were conducted. During the neutralization, the transfer stuff was started to set up, including Whatman paper, tissue paper, a big glass, a baking dish with 10X SSC buffer, and blotting membrane. The gel was rinsed with 10X SSC buffer for a while, then set to transfer DNA to blotting membrane overnight with gravity. After the DNA transfer is done, the membrane was rinsed by $2 \mathrm{X}$ SSC buffer to remove some residual gel, and placed on Whatman paper for a while for drying. The membrane was then UV crosslinked by Stratalinker "autocross link" setting (STRATAGENE).

The UV crosslinked membrane was set into a hybridization tube with Hybrid Mix buffer, and placed in a hybridization oven at $65^{\circ} \mathrm{C}$ for at least $30 \mathrm{~min}$. Meanwhile, radioactive WPRE probes were generated from 50 ng PCR products using the Rediprime ${ }^{\mathrm{TM}}$ || DNA Labeling System (GE Healthcare) and [a-32P]-dCTP (PerkinElmer), according the manufacturers' protocols. Radio-labeled WPRE probes were purified using the ProbeQuant G-50 Micro Columns (Amersham Pharmacia Biotech, 27-5335-01) and heat-denatured $\left(99^{\circ} \mathrm{C}, 5 \mathrm{~min}\right.$ followed by 5 min on ice). Hybridization was performed at $65^{\circ} \mathrm{C}$ overnight in Hybrid Mix with slow rotation. The following day, the membrane was washed $3 \mathrm{x}$ in $10 \mathrm{XSSC}$ buffer at $65^{\circ} \mathrm{C}$, wrapped with Saran Wrap and exposed to film (Kodak) for several days at $-80^{\circ} \mathrm{C}$. 


\subsubsection{Transcriptome Analysis}

The gene expression filing of hiPSC derived neural precursors was carried out by the Transkriptomanalyselabor (TAL) -Microarray and Deep-Sequencing Facility- in University of Göttingen. Neural precursors were collected by Accutase, suspended in TRIzol ${ }^{\circledR}$ Reagent (Life Technologies, $15596-026)$, and frozen at $-80^{\circ} \mathrm{C}$. The total RNA was isolated and assessed RNA quality by the Agilent RNA 6000 Nano Kit (Agilent, 5067-1511). Following the standard procedure, the resulting gene expression data was obtained using Human GE $4 \times 44 \mathrm{~K}$ V2 Microarray (Agilent, G2519F-026652).

\subsubsection{Functional Assays}

\subsubsection{Electrophysiology}

Electrophysiological analyses were performed by Dr. Kun-Han Lin in the Max-Planck Institute for Biophysical Chemistry. Patch-clamp recordings were obtained from differentiated cells using an EPC-10 amplifier controlled by Pulse software (HEKA Elektronik, Lambrecht/Pfalz, Germany). Cells were visualized by differential interference contrast microscopy through a 60x water-immersion objective (NA 1.0, Olympus, Hamburg, Germany) using an Axioskop FS microscope (Zeiss, Oberkochen, Germany). All experiments were performed at room temperature.

Patch pipettes were made from borosilicate glass (Science Products GmbH, Hofheim, Germany). Pipette resistance was 4-6 M . Access resistance (Rs) values were $\leq 20 \mathrm{M} \Omega$ for recordings. Rs was compensated 60-70\% during voltage-clamp experiments. For measuring sodium (INa(V)), potassium currents (IKa(V)), and action potentials (APs), pipettes were filled with K-gluconate based solution (Lin, Oleskevich, \& Taschenberger, 2011).

\subsubsection{Carbonyl cyanide m-chlorophenyl hydrazine (CCCP) Treatments}

The mitochondrial stress experiments were carried out by Dr. Susann Kummer in the Department of NanoBiophotonics, Max-Planck Institute for Biophysical Chemistry. The cells were exposed to CCCP (Fluka BioChemika, 21855), which integrates into the inner membrane of mitochondria leading to an uncoupling of the proton gradient, and thus, to cellular ATP depletion and cell death. Incubation of hiPSC-derived neurons with 100-200 $\mu \mathrm{M}$ CCCP for $45 \mathrm{~min}$ at $37^{\circ} \mathrm{C}$, was followed by fixation using $4 \%$ paraformaldehyde, blocking of 
unspecific bindings using 5\% BSA, and cell membrane permeabilization using $0.1 \%$ Triton $\mathrm{X}$-100. (Incubation was at room temperature for $5 \mathrm{~min}$ for each step). Cells subjected to microscopic imaging were treated with specific antibodies in a double staining procedure. Immunostaining was performed using the antibody combinations summarized in table 2.2 \& 2.4 for $1 \mathrm{~h}$ at room temperature to visualize mitochondria and dopaminergic neurons. The fluorescent images were acquired by using Leica DM6000 widefield microscope.

\subsubsection{Intracellular ROS Assay}

The cell-permeant 2', 7'-dichlorodihydrofluorescein diacetate (H2-DCFDA) is known as a general oxidative stress indicator for detection of intracellular ROS (Reactive Oxygen Species). The acetate groups of DCFDA are cleaved by intracellular esterases and oxidation, and fluorescent fluorophore, DCF, is yielded. Therefore, the level of ROS can be measured by the intensity of fluorescent DCF.

The hiPSC-derived neurons were exposed $100 \mathrm{mM} \mathrm{H}_{2} \mathrm{O}_{2}$ for $1 \mathrm{~h}$ at $37^{\circ} \mathrm{C}$ and followed by incubation with $10 \mu \mathrm{M}$ DCFDA for additional $20 \mathrm{~min}$. Afterwards, cells were harvested and suspended in HEPES buffer. The fluorescence of living cells was monitored using a flow cytometer (BD FACSCanto II). The excitation and emission wavelengths used for the dyes are $\lambda e x / \lambda e m=475 / 525 \mathrm{~nm}$ for DCF. The data was analyzed using flowing software (http://www.flowingsoftware.com). 


\subsection{Material}

\subsubsection{Medium \& Reagents}

\subsubsection{Culture Medium}

\section{MEF Medium/ hFB Medium/ 293T Medium}

High D-MEM (Invitrogen, 41965-039)

$10 \%$ FCS (Fetal Calf Serum)

1x P/S (Penicillin-Streptomycin, Liquid)(Invitrogen, 15140-122)

\section{hPSC Medium}

Knockout D-MEM (Invitrogen, 10829-018)

20\% Knockout Serum Replacement (KSR) (Invitrogen, 10828-028)

1x NEAA (Non-Essential Amino Acids Solution 10 mM, 100x) (Invitrogen, 11140-050)

2 mM L-Glutamine (Invitrogen, 25030-024)

$0.1 \mathrm{mM} \beta$-Mercaptoethanol (Sigma, M3148)

$1 \times \mathrm{P} / \mathrm{S}$

\section{Differentiation Medium}

Knockout D-MEM (KO-DMEM)

$20 \%$ FCS

1x NEAA

$1 \times \mathrm{P} / \mathrm{S}$

2 mM L-Glutamine

$0.1 \mathrm{mM} \beta$-Mercaptoethanol

\section{Neural Induction Medium}

Knockout D-MEM

15\% Knockout Serum Replacement

1x NEAA

$1 \times \mathrm{P} / \mathrm{S}$

2 mM L-Glutamine

$0.1 \mathrm{mM} \beta$-Mercaptoethanol 


\section{Neural Precursor Medium (NP Medium)}

Neurobasal Medium (Invitrogen, 21103-049)

1x NEAA

$1 \times \mathrm{P} / \mathrm{S}$

2 mM L-Glutamine

1x N2 (Invitrogen, 17502-048)

1x B27 Minus Vitamin A (Invitrogen, 12587-010)

0.2 mM Ascorbic Acid (Sigma, A4403)

\section{Neuronal Differentiation Medium}

Neurobasal Medium

1x NEAA

$1 \times \mathrm{P} / \mathrm{S}$

2 mM L-Glutamine

1x B27 Minus Vitamin A

$0.2 \mathrm{mM}$ Ascorbic Acid

\section{PA6 Medium}

MEM $\alpha$ medium, Nucleosides (Invitrogen, 12571-063)

$10 \%$ FCS

$1 \times \mathrm{P} / \mathrm{S}$

\section{FP Induction Medium}

50\% Knockout D-MEM

$50 \%$ Neurobasal Medium

1x NEAA

$1 \times \mathrm{P} / \mathrm{S}$

2 mM L-Glutamine

$1 \times N 2$

1x B27 Minus Vitamin A 


\subsubsection{Growth Factors, Recombinant Protein \& Small molecules}

bFGF (PeproTech, 100-18B)

Y27632 (TOCRIS, 1254)

Noggin (R\&D SYSTEMS, 344-NG)

EGF (PeproTech, AF-100-15)

Dorsomorphin (Sigma, P5499)

FGF8 (PeproTech, 100-25)

Shh (PeproTech, 100-45)

BDNF (PeproTech, 450-02)

GDNF (PeproTech, 450-10)

TGF- $\beta 3$ (R\&D SYSTEMS, 243-B3-002)

dbcAMP (Sigma, D0627)

DAPT (TOCRIS, 2634)

SB431542 (TOCRIS, 1614)

LDN193189 (STEMGENT, 04-0074)

Shh C24II (R\&D SYSTEMS, 1845-SH)

FGF20 (PeproTech, 100-41)

purmorphamine (Cayman Chemical, 10009634)

CHIR 99021 (STEMGENT, 04-0004)

Poly-D-Lysine (Sigma, P6407)

Laminin (Sigma, L2020)

Fibronectin (R\&D SYSTEMS, 1030-FN)

H2-DCFDA (Sigma, D6883)

\subsubsection{Buffers \& Reagents}

\section{NTMT Solution}

$100 \mathrm{mM} \mathrm{NaCl}$

100 mM Tris- $\mathrm{HCl} \mathrm{pH} 9.5$

$50 \mathrm{mM} \mathrm{MgCl} 2$

1\% Tween 20 
DNA Lysis Buffer

100 mM Tris- $\mathrm{HCl} \mathrm{pH} 8.0$

5 mM EDTA

$0.2 \%$ SDS

$200 \mathrm{mM} \mathrm{NaCl}$

1X Neutralization Buffer

$1.5 \mathrm{M} \mathrm{NaCl}$

$0.5 \mathrm{M}$ Tris- $\mathrm{HCl} \mathrm{pH} 7.5$

\section{X Denaturation Buffer}

$0.5 \mathrm{M} \mathrm{NaOH}$

$1.5 \mathrm{M} \mathrm{NaCl}$

20X SSC Buffer

$3 \mathrm{M} \mathrm{NaCl}$

$0.3 \mathrm{M} \mathrm{Na}$ Citrate

Hybrid Mix

500 mM Sodium Phosphate Buffer, PH 7.2

$7 \%$ SDS

1 mM EDTA

\section{HEPES Buffer}

$5 \mathrm{mM} \mathrm{KCl}$

$140 \mathrm{mM} \mathrm{NaCl}$

$2 \mathrm{mM} \mathrm{CaCl}_{2}$

$1 \mathrm{mM} \mathrm{MgCl} 2$

10 mM Glucose

$5 \mathrm{mM}$ Hepes 


\subsubsection{Plasmids}

Table 2.1: List of Plasmids used

\begin{tabular}{lll}
\hline Function & Plasmids & Source \\
\hline \hline Virus Production & pMD2.G & Addgene, 12259 \\
Virus Production & psPAX2 & Addgene, 12260 \\
Reprogramming & pHAGE2 EF1aL-hSTEMCCA-W-loxP & Gift from Kotton Lab \\
Cre Excision & pHAGE2-Cre-IRES-PuroR & Gift from Kotton Lab \\
Bisulfite sequencing & pTZ57R/T Cloning Vector & Fermentas, K1214
\end{tabular}

Kotton Lab: Pulmonary Center, Boston University School of Medicine

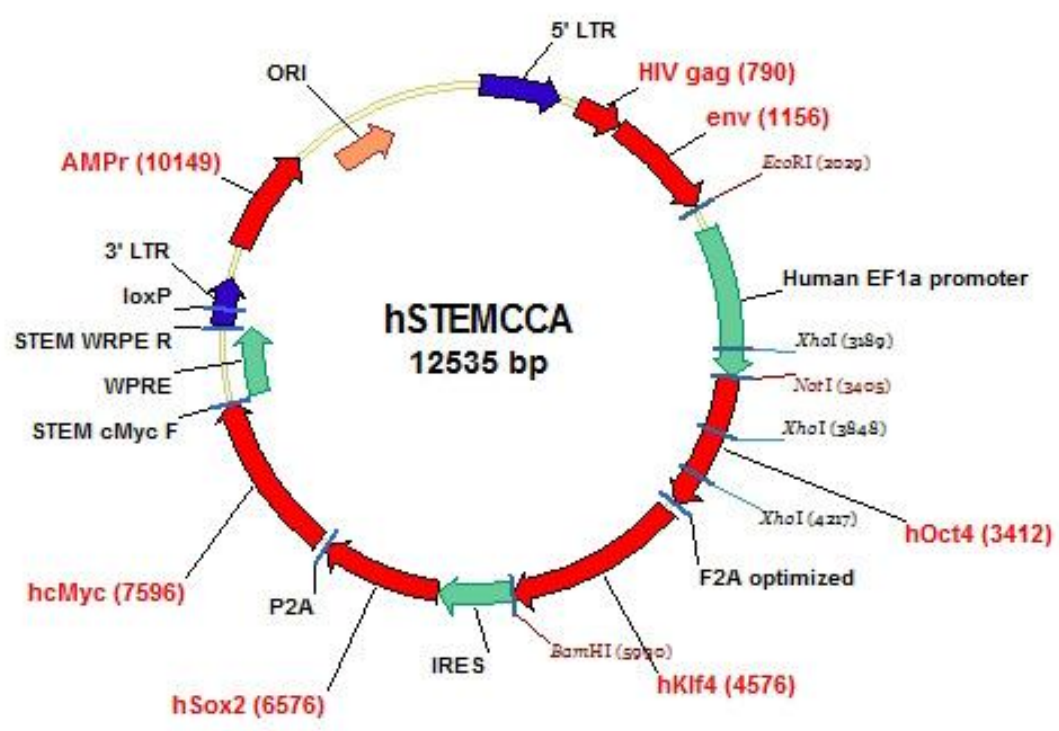

Figure 2.1: The Map of Lentiviral Vector hSTEMCCA.

(pHAGE2 EF1aL-hSTEMCCA-W-loxP)

\subsubsection{Primary Antibodies}

Table 2.2: List of Primary Antibodies used

\begin{tabular}{llll}
\hline Antibody & Species & Dilution & Source \\
\hline \hline Oct4 & Rabbit Polyclonal & $1: 500$ & Abcam, ab19857 \\
Sox2 & Rabbit Polyclonal & $1: 400$ & Abcam, ab15830 \\
Nanog & Rabbit Polyclonal & $1: 100$ & Abcam, ab21624 \\
Nanog & Goat Polyclonal & $1: 100$ & R\&D Systems, AF1997
\end{tabular}




\begin{tabular}{|c|c|c|c|}
\hline $\operatorname{Lin} 28$ & Rabbit Polyclonal & 1: 750 & Abcam, ab63740 \\
\hline TRA-1-60 & Mouse Monoclonal & 1: 100 & Abcam, ab16288 \\
\hline SSEA4 & Mouse Monoclonal & 1: 100 & Abcam, ab16287 \\
\hline Sox17 & Goat Polyclonal & 1: 100 & R\&D Systems, AF1924 \\
\hline Brachyury & Goat Polyclonal & 1: 100 & R\&D Systems, AF2085 \\
\hline$\alpha$-Fetoprotein/AFP & Mouse Monoclonal & 1: 100 & R\&D Systems, MAB1368 \\
\hline Tyrosine Hydroxylase/TH & Rabbit Polyclonal & 1: 300 & Millipore, AB152 \\
\hline$\beta$-tubulin III/Tuj1 & Mouse Monoclonal & 1: 1000 & Covance, MMS-435P \\
\hline Nestin & Mouse Monoclonal & 1: 750 & R\&D Systems, MAB1259 \\
\hline Msi1 & Rabbit Polyclonal & 1: 200 & Abcam, 21628 \\
\hline Pax6 & Rabbit Polyclonal & 1: 300 & Covance, PRB278P \\
\hline FoxA2 & Goat Polyclonal & 1: 100 & Santa Cruz, sc-6554 \\
\hline $\operatorname{Lm} \times 1 A$ & Rabbit Polyclonal & 1: 2000 & Millipore, AB10533 \\
\hline EN1 & Mouse Monoclonal & 1: 50 & DSHB, 4 G11 \\
\hline TOM20 & Mouse Monoclonal & 1: 100 & BD Bioscience, 612278 \\
\hline
\end{tabular}

DSHB: Developmental Studies Hybridoma Bank

\subsubsection{Secondary Antibodies}

Table 2.3: List of Secondary Antibodies used

\begin{tabular}{lllll}
\hline Conjugate & Host species & Reactivity & Dilution & Source \\
\hline \hline Alexa 488 & Donkey & Rabbit & $1: 750$ & Invitrogen, A-21206 \\
Alexa 488 & Goat & Rabbit & 1: 750 & Invitrogen, A-11008 \\
Alexa 488 & Goat & Rabbit & 1: 100 & Molecularprobes, A11034 \\
Alexa 488 & Chicken & Goat & $1: 750$ & Invitrogen, A-21467 \\
Alexa 488 & Goat & Mouse & $1: 750$ & Invitrogen, A-11001 \\
Alexa 594 & Chicken & Goat & $1: 750$ & Invitrogen, A-21468 \\
Alexa 594 & Donkey & Rabbit & $1: 750$ & Invitrogen, A-21207 \\
Alexa 594 & Goat & Rabbit & $1: 750$ & Invitrogen, A-11012 \\
Alexa 594 & Donkey & Mouse & $1: 750$ & Invitrogen, A-21203 \\
Alexa 594 & Goat & Mouse & $1: 750$ & Invitrogen, A-11005 \\
Rhodamine & Sheep & Mouse & $1: 100$ & Dianova \\
\hline
\end{tabular}


Rhodamine labeling anti-mouse KK114 was made in the Department of NanoBiophotonics (Kolmakov et al., 2010).

\subsubsection{PCR Primers used for Cre Excision}

Table 2.4: List of PCR Primers for Cre Excision

\begin{tabular}{ll}
\hline Gene/ Oligos & Primer sequence 5'-3' \\
\hline \hline STEM_SPM_F & GCCGCCCCCAGCAGACTTCA \\
STEM_SPM_R & GGGGTGGGCAGCAGCTCGAA \\
WPRE_F & CGTAAGTCGATAGATCCTAATCAAC \\
WPRE_R & TTAAAGGTACCAGGCGGGGAG \\
\hline
\end{tabular}

\subsubsection{PCR Primers used for Fingerprinting}

Table 2.5: List of PCR Primers for Fingerprinting

\begin{tabular}{ll}
\hline Gene/ Oligos & Primer sequence 5'-3' \\
\hline \hline D21S2055_F & AACAGAACCAATAGGCTATCTATC \\
D21S2055_R & TACAGTAAATCACTTGGTAGGAGA \\
DS17S1290_F & GCAACAGAGCAAGACTGTC \\
DS17S1290_R & GGAAACAGTTAAATGGCCAA \\
D7S796_F & TTTTGGTATTGGCCATCCTA \\
D7S796_R & GAAAGGAACAGAGAGACAGGG \\
D10S1214_F & ATTGCCCCAAAACTTTTTTG \\
D10S1214_R & TTGAAGACCAGTCTGGGAAG \\
\hline
\end{tabular}

\subsubsection{PCR Primers used for Pluripotency}

Table 2.6: List of PCR Primers for Pluriopotency

\begin{tabular}{ll}
\hline Gene/ Oligos & Primer sequence 5'-3' \\
\hline \hline OCT4_F & CAGTGCCCGAAACCCACAC \\
OCT4_R & GGAGACCCAGCAGCCTCAAA \\
SOX2_F & TTACCTCTTCCTCCCACTCCAG \\
SOX2_R & GGGTTTTCTCCATGCTGTTTCT
\end{tabular}




$\begin{array}{ll}\text { NANOG_F } & \text { CCTGAAGACGTGTGAAGATGAG } \\ \text { NANOG_R } & \text { GCTGATTAGGCTCCAACCATAC } \\ \text { TERT_F } & \text { GTGAACAGCCTCCAGACGGT } \\ \text { TERT_R } & \text { GCGTTCTTGGCTTTCAGGAT } \\ \text { FOXD3_F } & \text { AAGCCCAAGAACAGCCTAGTGA } \\ \text { FOXD3_R } & \text { GGGTCCAGGGTCCAGTAGTTG } \\ \text { TDGF1_F } & \text { TACCTGGCCTTCAGAGATGACA } \\ \text { TDGF1_R } & \text { CCAGCATTTACACAGGGAACAC } \\ \text { GAPDH_F } & \text { TGACATCAAGAAGGTGGTGAAGC } \\ \text { GAPDH_R } & \text { CCCTGTTGCTGTAGCCGTATTC }\end{array}$

\subsubsection{PCR Primers used for Bisulfite OCT4 Promoter}

Table 2.7: List of PCR Primers for Bisulfite OCT4 Promoter

\begin{tabular}{ll}
\hline Gene/ Oligos & Primer sequence 5'-3' $^{\prime}$ \\
\hline \hline hOCT4P-4_F & GGATGTTATTAAGATGAAGATAGTTGG \\
hOCT4P-4_R & CCTAAACTCCCCTTCAAAATCTATT \\
\hline
\end{tabular}

\subsubsection{PCR Primers used for QRT-PCR}

Table 2.8: List of PCR Primers for QRT-PCR

\begin{tabular}{ll}
\hline Gene/ Oligos & Primer sequence 5' -3' $^{\prime}$ \\
\hline \hline GAPDH_F & TGACATCAAGAAGGTGGTGAAGC \\
GAPDH_R & CCCTGTTGCTGTAGCCGTATTC \\
FGF20_F & GACCACAGCCTCTTCGGTAT \\
FGF20_R & CTGCTCCCTAAAGATGCATTCG \\
\hline
\end{tabular}




\section{Results}

\subsection{Recruitment of PD Patients}

In collaboration with Prof. Dr. Claudia Trenkwalder in Paracelsus-Elena-Klinik, Kassel, Germany, and following German ethical regulations, PD patients were recruited with the purpose of establishment of PD patient specific hiPSC model and investigation of the PD pathogenesis. At the start, 6 PD patients who developed obvious PD symptoms at the age of 38 54 years were enlisted and agreed to perform skin punch biopsy. However, only 4 PD patients' dermal fibroblasts could be isolated and derived from the skin biopsies. Together with 2 controls contributed by healthy persons, 3 PD patients' fibroblasts were further used for generation of hiPSC model and direct differentiation of dopaminergic neurons. The clinical information of 3 PD patients was listed in Table 3.1.

Table 3.1: Clinical Information of PD Patients

\begin{tabular}{|c|c|c|c|}
\hline PD Patient & Kas01 & Kas02 & Kas05 \\
\hline Gender & female & male & female \\
\hline Age of Onset & 35 & 44 & 41 \\
\hline Age of Skin Biopsy & 38 & 47 & 48 \\
\hline Family History & N/A & N/A & $\begin{array}{l}\text { mother and maternal } \\
\text { grandfather with PD }\end{array}$ \\
\hline Features & pronounced dyskinesias & $\begin{array}{l}\text { pronounced dyskinesias, } \\
\text { DBS }\end{array}$ & \\
\hline Clicical Symptoms & $\begin{array}{l}\text { freezing, rigid, } \\
\text { dysarthria,dystonia,left- } \\
\text { sided hypokineseia, } \\
\text { severe fluctuations }\end{array}$ & $\begin{array}{l}\text { tremor and left-sided } \\
\text { hypokinesia, motor } \\
\text { fluctuations }\end{array}$ & $\begin{array}{l}\text { rigid, dystonia, RLS, right- } \\
\text { sided hypokinesia, } \\
\text { fluctuations }\end{array}$ \\
\hline UPDRS III & $22 / 46$ & $37 / 77$ & $10 / 35$ \\
\hline Levodopa Response & yes & yes & yes \\
\hline Present Treatments & $\begin{array}{l}\text { Amantsdin, Apomorphin, } \\
\text { levodopa }\end{array}$ & $\begin{array}{l}\text { DBS, Levodopa, } \\
\text { Entacapon, Rasagilin, } \\
\text { Ropinirol }\end{array}$ & $\begin{array}{l}\text { Levodopa, Ropinirol, } \\
\text { entacapon, amantadin }\end{array}$ \\
\hline
\end{tabular}

Note: UPDRS III (Unified Parkinson's Disease Rating Scale-motor examination), RLS (Restless legs syndrome), DBS (Deep Brain Stimulation), N/A (Not Available) 


\subsection{Generation of PD Patient Specific Induced Pluripotent Stem Cells}

\subsubsection{Reprogramming of PD patients' Fibroblasts to Pluripotent States}

To generate human iPSCs, the protocol used in this study was adopted from Kotton and Mostoslavsky's published article. (Somers et al., 2010) They generated a humanized version of lentiviral Stem Cell Cassette (hSTEMCCA) (Figure 3.1.A) containing four defined factors (Oct4, KIf4, Sox2, and c-Myc). In addition, this lentiviral vector is flanked by a loxP site on 3' long terminal repeat (LTR) region. Upon proviral replication, the loxP site in the $3^{\prime}$ LTR is duplicated into the $5^{\prime}$ LTR, resulting in an integrated transgene flanked by loxP sites on both LTRs. The transgene would be then removed using the technology of Cre/loxP recombination.

Human dermal fibroblasts (Figure 3.2.a \& d) used for reprogramming were within passage 4. (Figure 3.1.B) One day before transduction (day 0), 1x105 human fibroblasts were plated in hFB medium. The next day (day 1 ), the cells were infected with hSTEMCCA lentivirus. After $24 \mathrm{~h}$ of transduction (day 2), the virus remaining in the medium was removed, and the cells were fed with fresh hFB medium for 2 days. On day 5, the transduced fibroblasts were passaged onto inactive mouse embryonic fibroblast (MEF) feeder layers. Acting as normal human embryonic stem (hES) cell culture, the cells were fed with hPS medium supplemented with bFGF. In the first week (day 5-12), the cells were treated with sodium butyrate ( $\mathrm{Na}$ butyrate) additionally. It has been reported that $\mathrm{Na}$ butyrate, as a Histone deacetylase inhibitor, greatly enhances the efficiency of hiPSC derivation (Mali et al., 2010). Afterwards, the cells were kept growing on the hPS medium. About day 20-25, some small human ES-like colonies, started to appear in the dish. Following one month of transduction, hiPSC colonies with the appropriate size for propagation in culture were passaged. These hiPSC colonies were isolated and picked up mechanically under the stereomicroscope in the laminar flow hood. Isolated colonies were transferred to a fresh MEF feeder plate respectively and propagated under standard hES cell culture condition. Unlike parental fibroblasts, fully reprogrammed colonies were easily distinguishable by their similarity to hESC colonies and their tightly packed morphology. All selected clones had high nuclei to cytoplasm ratio, well defined borders, densely packed colonies, and were indistinguishable from human ESCs. (Figure 3.2.b \& e) In addition, those hiPSC clones showed the enzyme activity of alkaline phosphatase (ALP), one of the characteristics of hESC. (Figure $3.2 \mathrm{c} \& \mathrm{f}$ ) 
For each sample, up to 30 hiPSC clones were picked primarily. 1-3 clones per sample were continuously passaged; the rest of them were frozen in the liquid nitrogen tank.

\subsubsection{Excision of Transgene from Host Genome of Human ES-like Clones by Cre/loxP Recombination}

The previous reports have shown that presences of transgenic reprogramming factors would interfere with cellular activities of iPSCs. For example, constitutive expression of reprogramming transgenes interrupts the ability of mouse iPSC differentiation into different germ layers. (Sommer et al., 2010) The remaining integrated transgenic factors could also cause tumor formation and affect the global genetic network (Okita, Ichisaka, \& Yamanaka, 2007) (Soldner et al., 2009). Therefore, it is imperative for developing an improved method for generating human iPSC free of transgene integration. The lentiviral hSTEMCCA used in this work is a multicistronic vector flanked with a loxP site next to the transgenes. Use of this single vector allows deriving human iPSC with a single excisable viral integration, and further efficiently generating factor-free hiPSCs followed by Cre-mediated excision.

In order to excise the transgenic hSTEMCCA from the generated hiPSC clones, the plasmid pHAGE2-Cre-IRES-PuroR, ectopic expression of Cre recombinase and puromycin resistant plasmid, was delivered into the hiPSC clones for transient expression of Cre recombinase within cells. Besides, the cells were exposed to puromycin antibiotic selection for 2 days. Afterwards, the cells would recover and grow for 1-2 weeks till new surviving colonies mature for pick-up. Those pick-up subclones were cultivated, expanded, and then examined for transgene excision by PCR primarily. For each hiPSC clone, at least 7 subclones were picked. Deletion of hSTEMCCA vector was nearly $100 \%$ successful in these screened subclones. (Figure 3.3.A) Furthermore, the absence of integrated hSTEMCCA or Cre plasmid was evident using the technology of Southern blot probed with the same element (WPRE) in both vectors. (Figure 3.3.B)

In summary, human dermal fibroblasts isolated from 2 controls (wt \& Hess) and 3 PD patients (Kas01, Kas02 \& Kas05) were dedicated to generating hiPSCs using the single lentiviral vector hSTEMCCA. Beyond the excision of transgene, 2 hiPSC clones for each control (wt 1, wt 7 \& Hess 6, Hess 9) and PD (Kas01 19, Kas01 21 \& Kas02 14, Kas02 23 \& 
Kas05 5, Kas05 29) samples were employed for the characterization of hiPSC and directed differentiation of neural precursor and dopaminergic neurons.
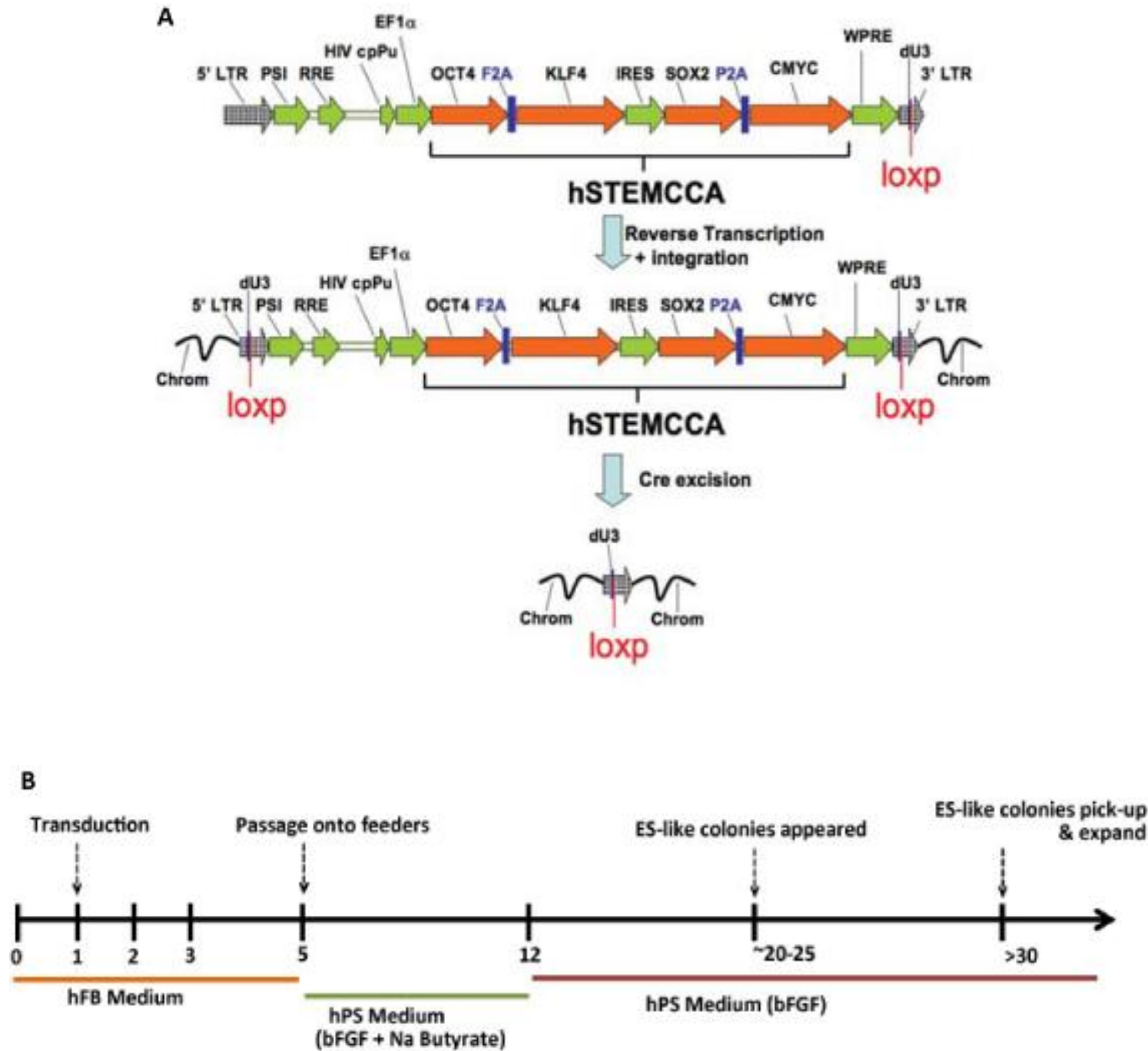

Figure 3.1 Generation of Human iPSC by a Lentiviral hSTEMCCA Vector.

(A) Human fibroblasts were reprogrammed by lentiviral hSTEMCCA-loxP vector encoding 4 defined factors (Oct4, KIf4, Sox, and c-Myc). The integrated transgene could be excised from the host genome by Cre/loxP recombination. The picture was adopted from (Somers, et al., 2010). (B) Time flow of reprogramming in this study. 


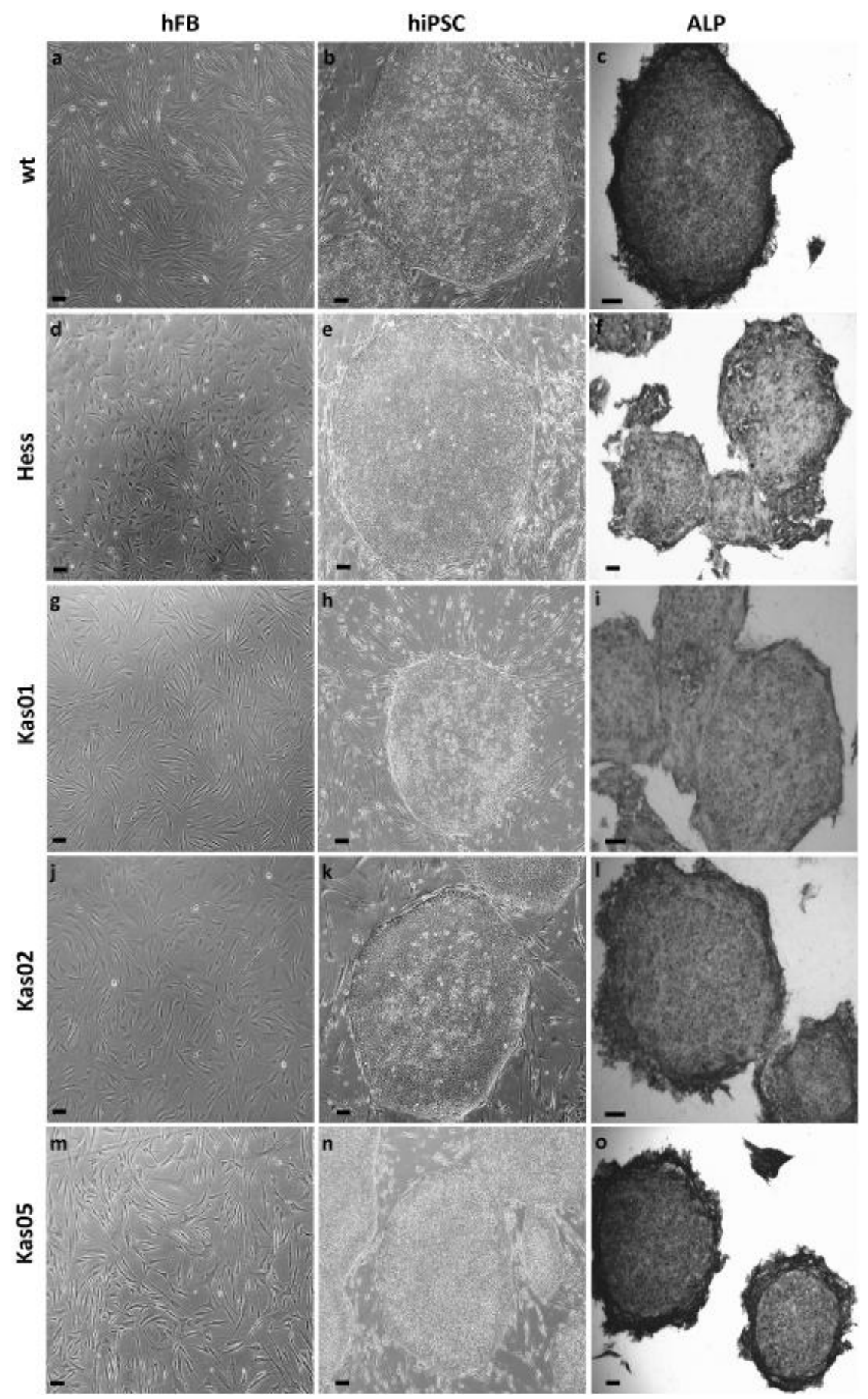

Figure 3.2: Cell Morphology of Human Skin Fibroblasts and Generated hiPSC Clones.

Cell morphologies of primary human fibroblasts were control wt (a) \& Hess (d) and PD patient Kas01 (g), Kas02 (j) \& $\operatorname{Kas05~(m).~(b),~(e),~(h),~(k)~\& ~(n)~were~hiPSC~clones~derived~}$ from control and PD patients grown on feeder layers, respectively. Alkaline phosphatase (ALP) activity was examined for derived hiPSC clones (c, f, l, j \& o). Black bar represents 100 $\mu \mathrm{m}$. 

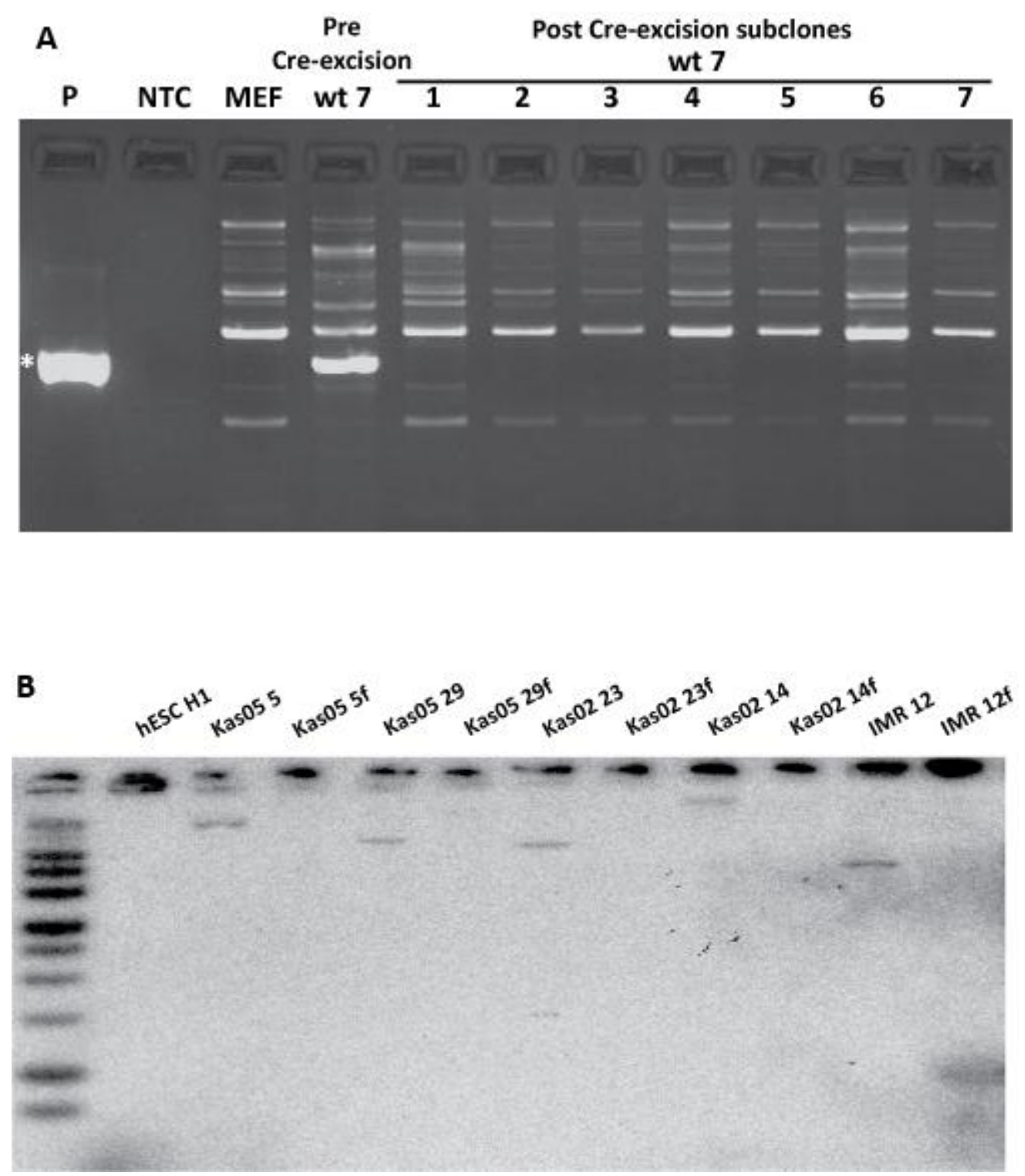

Figure 3.3: Screen of Transgene-free hiPSC Clones by PCR and Southern Blot.

(A) Preliminary screens were done by PCR using the plasmid specific primer set (SPM). P was diluted plasmid as positive control. 1 7 were post-excision subclones picked from hiPSC wt 7 clones. Note: MEF (feeder cells), NTC (non template control).

(B) Southern blot further confirmed that clone kas05 5f, Kas05 29f, kas02 23f, Kas02 $14 f$ \& IMR $12 \mathrm{f}$ were transgenic-free excised form clone Kas05 5, Kas05 29, Kas02 23, Kas02 14 \& IMR 12, respectively. hESC H1 cell line was negative control. 


\subsubsection{Characterization of PD Patient Specific hiPSC Lines}

Overexpression of pluripotency-associated transcription factors, like Oct4, Sox2, Klf4, c-Myc, and/or Nanog \& Lin28, convert human somatic cells (e.g. fibroblast or keratinocyte) to the undifferentiated state, termed human induced pluripotent stem cells (hiPSCs). hiPSC is not only indistinguishable from hESC in morphology but also exhibits the specific characteristics of hESC. Besides the demonstration of their genetic origin from the parental cell, hiPSC is supposed to meet several criteria: (1) unlimited proliferation; (2) expression of pluripotency markers and surface antigen of hESC, such as Oct4, Nanog \& SSEA4, Tra-1-60; (3) potential of differentiation in vitro to three germ layers (ectoderm, mesoderm, and endoderm); (4) competence of teratoma formation in vivo when injected into immune-compromised mice. Higher standards for pluripotency are that pluripotent stem cells (PSCs) are competent for chimera contribution, germline transmission, and tetraploid complementation, routinely examined in murine iPSCs (Chen \& Liu, 2009). However, due to ethical restriction, these stringent tests cannot be applied to the assessment of human pluripotent stem cells. In this work, a series of pluripotency tests would be examined in the generated PD patient specific hiPSCs (PD-hiPSCs).

\section{DNA Fingerprinting}

In order to verify the origins of control/ PD-hiPSCs, the PCR-based fingerprinting analysis was set up. The genomic DNA was isolated from respective parental fibroblasts and derived hiPSCs, and the genomic regions containing highly variable tetra-nucleotide repeats (VTNR) were amplified by designed PCR primers. Four different genomic loci were examined: D21S2055, repeat (GATA)n, average heterozygosity 0.88; D7S796, repeat (GATA)n, average heterozygosity 0.95; D10S1214, repeat (GGAA)n, average heterozygosity 0.97; and D17S1290, repeat (GATA)n, average heterozygosity 0.84 (Park, Arora, et al., 2008). The PCR amplified products were separated by agarose gel electrophoresis and shown the specific patterns among different parental fibroblasts and hiPSCs. By the combination of various sets of primers, the genetic relevance of derived hiPSC to the parental fibroblast was approved. Besides, the uncertainty of contamination of hESC cell line (e.g. H1) was excluded. (Figure 3.4) 


\section{Expression of hESC Specific Markers}

Control/PD-hiPSCs were further evaluated for the specific features of human pluripotent stem cells (hPSCs) molecular signature. Previously, it was shown that the derived hiPSCs resemble hESC in terms of morphology and alkaline phosphatase activity. (Figure 3.1) Moreover, the pluripotency associated markers, including Oct4, Sox2, Nanog, and Lin28, were detected in Control/PD-hiPSCs using immunocytochemistry. The hES cell-specific surface antigens, like SSEA4 and tumor-related antigen (TRA)-1-60, were assessed as well. (Figure 3.5.I) In addition, RT-PCR analysis showed that hiPSCs expressed a set of undifferentiated ES cell-marker genes at RNA level. Control/PD-hiPSC expressed endogenous OCT4, SOX2 \& NANOG with similar levels as seen in hESC cell line (H9). Besides, the presence of mRNA transcripts of reduced expression 1 (REX1), teratocarcinoma-derived growth factor 1 (TDGF1), and telomerase reverse transcriptase (hTERT) in control/PD-hiPSCs demonstrated the qualities of pluripotency regardless of the genetic condition represented within the parental fibroblasts. (Figure 3.5.II)

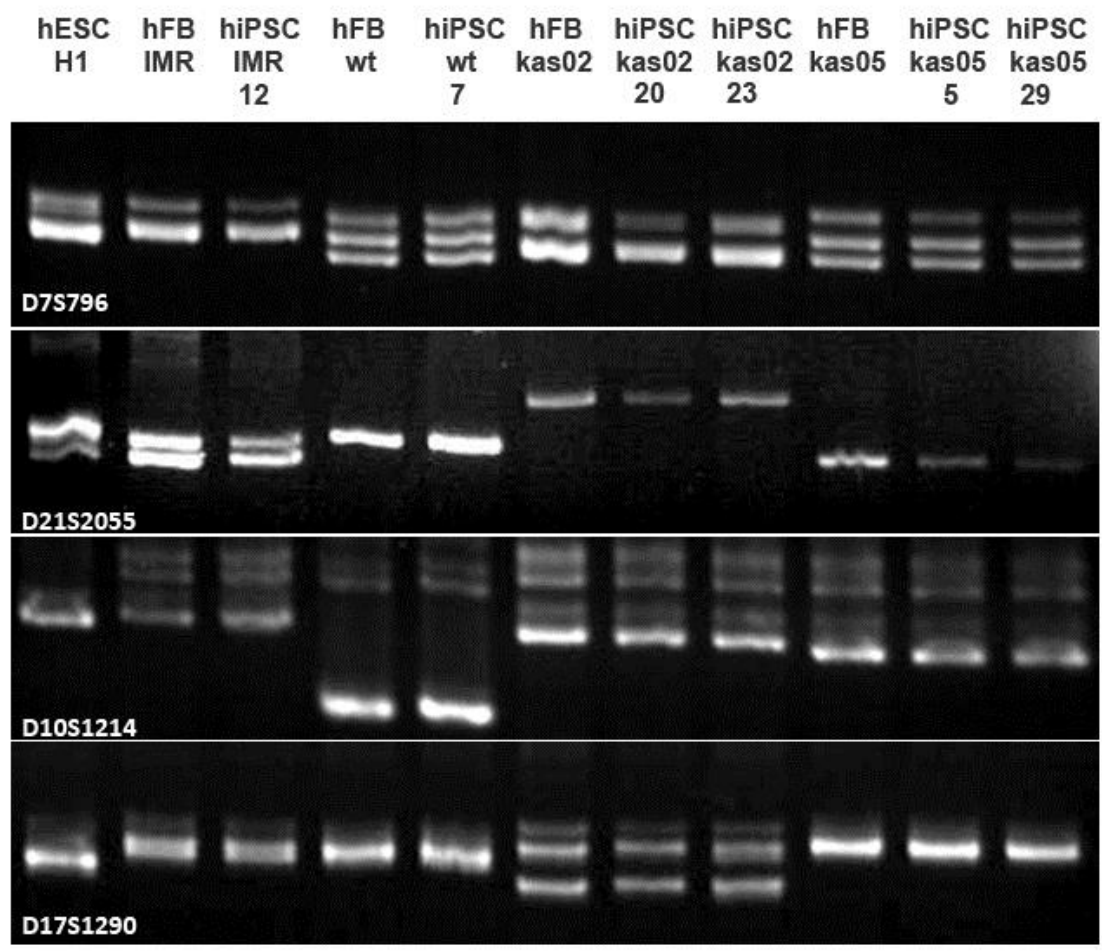

Figure 3.4: DNA Fingerprinting Analysis.

Genomic DNAs were prepared from hESC H9, IMR, control (wt) and PD patients (Kas02 \& Kas05) fibroblasts, and derived hiPSCs. DNA fingerprinting was analyzed by genomic PCR 
using designed primer sets (D7S796, D10S1214, D17S1290, and D21S2055). PCR products were subjected to $2.5 \%$ agarose gel electrophoresis.

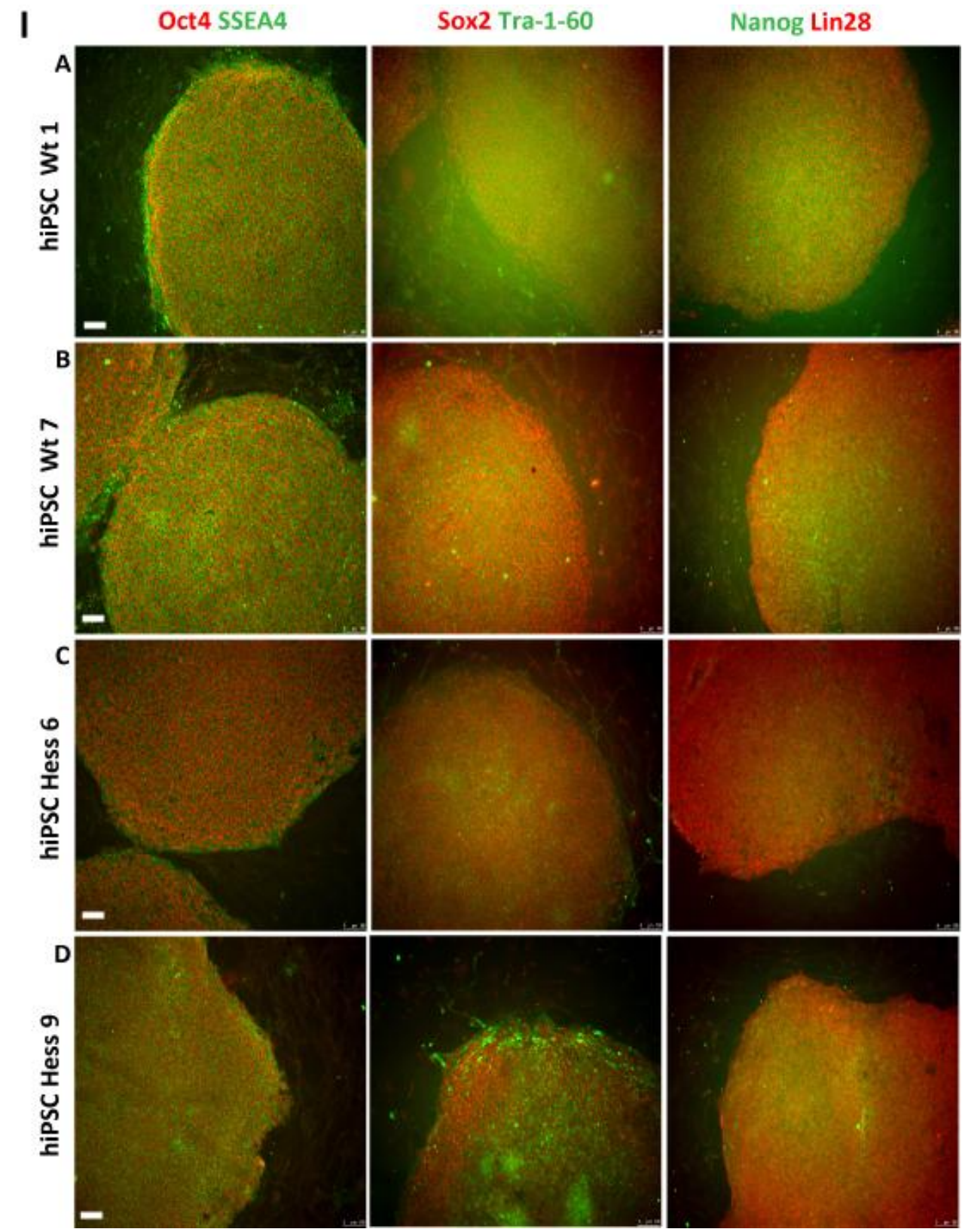




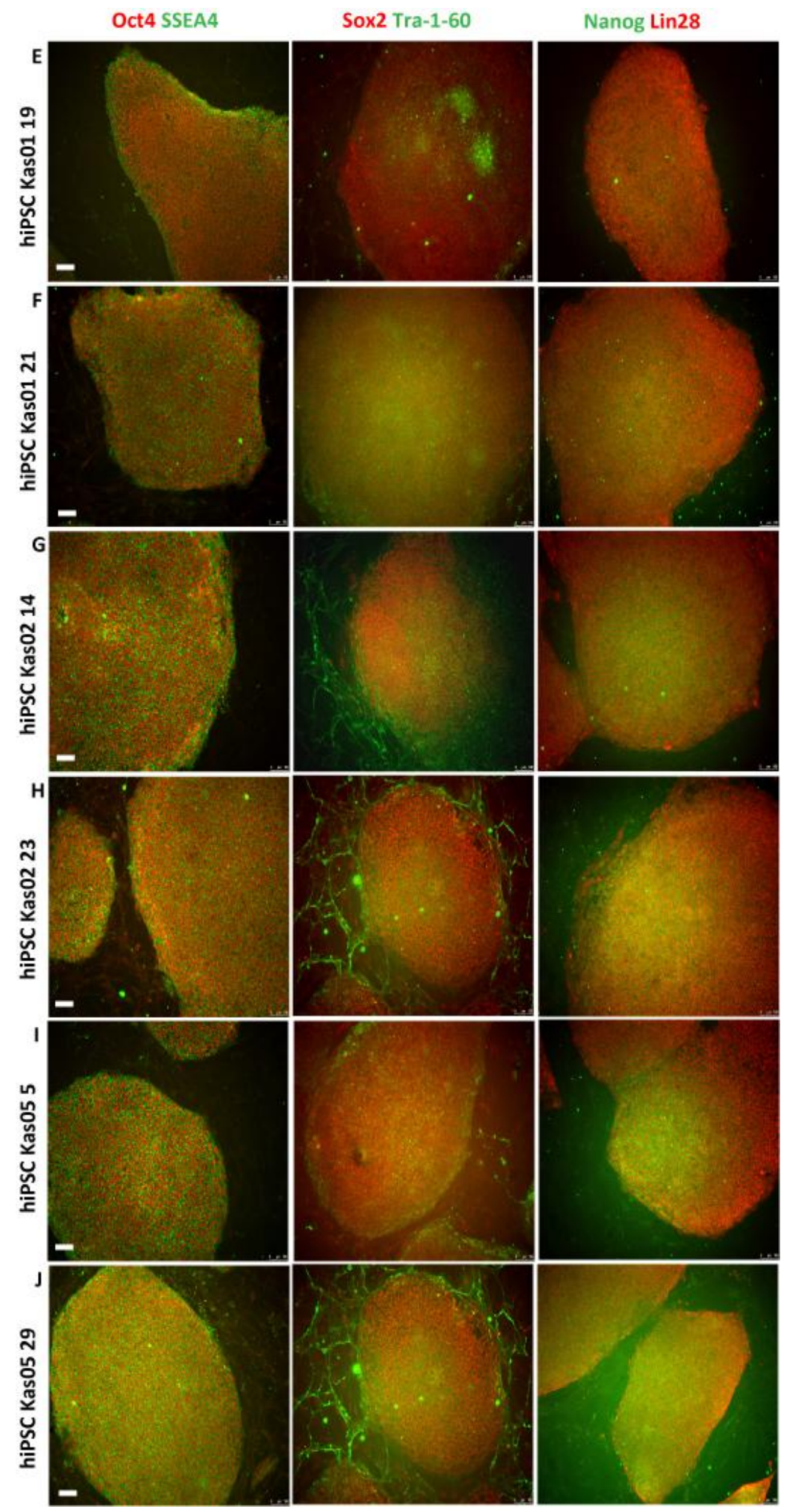




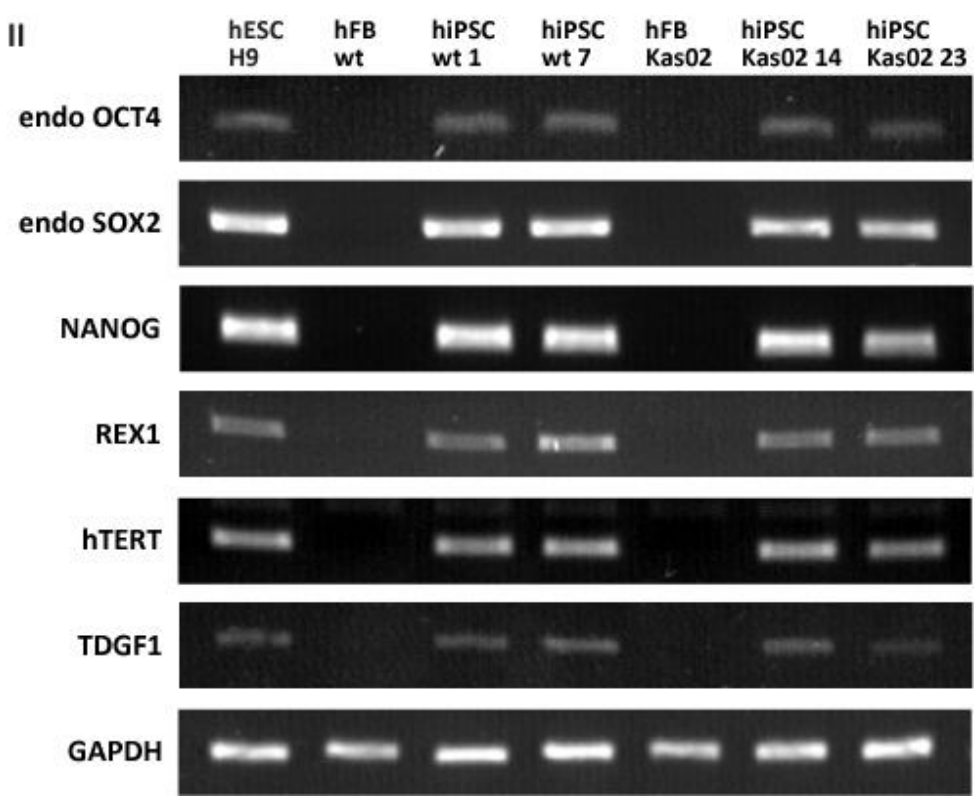

Figure 3.5: Detection of human ESC Markers in hiPSCs.

(I) Control (wt 1 (A), 7 (B) \& Hess 6(C), 9 (D)) and PD (Kas01 19 (E), 21 (F) \& Kas02 14 (G), 23 (H) \& Kas05 5(I), 29 (J)) hiPSC clones were examined for their pluripotency by immunostaining of Oct4, Sox2, Nanog \& Lin28, respectively. hES surface antigens, SSEA4 \& TRA-1-60, were present in those hiPSC cell lines as well. White bar represents $100 \mu \mathrm{m}$. (II) hES-related gene expressions (OCT4, SOX2, NANOG, REX1, hTERT, and TDGF1) were examined by RT-PCR. hESC H9 cell line was positive control, and hFB wt \& hFB KasO2 were negative controls. wt $1 \& 7$ and PD Kas02 $14 \& 23$ hiPSC clone displayed these pluripotent genes' expression.

\section{Methylation Analysis of the OCT4 Promoter Region}

It is believed that the iPSC generation involves the modifications of the epigenome during conversion of somatic cell to the pluripotent state. DNA methylation controls the genomic epigenetic state that regulates gene expression. Methylation $\left(-\mathrm{CH}_{3}\right)$ in a cytosine-phosphate-guanosine $(\mathrm{CpG})$ dinucleotide is a genetic alteration that favors genomic integrity, guarantees proper regulation of gene expression, and is required for long-term gene silencing. The reprogramming process has been shown to be associated with epigenetic changes (e.g. DNA hypomethylation) mostly on pluripotency-related gene promoters. Thus, the endogenous OCT4 and NANOG pluripotent genes may become reactivated in somatic cells undergoing faithful reprogramming. (Freberg, et al., 2007) 
To assess stable reprogramming of the epigenetic state of the OCT4 promoter in Control/PD-hiPSCs, the degree of DNA methylation of $5 \mathrm{CpG}$ dinucleotides in the OCT4 promoter (-2126 -1891 bp from the transcriptional start site TSS)) was examined. This was followed by bisulfite conversion of genomic DNA, and PCR amplification of the evaluated OCT4 promoter region. The amplified PCR product was cloned into the vector and sequenced. Sequences of at least 3 bacterial clones per genomic region examined are represented as rows of circles, with each circle symbolizing the methylation state of one CpG dinucleotide. (Figure 3.6) The results revealed that hiPSCs were highly unmethylated while CpG dinucleotides of the evaluated region were highly methylated in parental fibroblasts, indicating that the OCT4 promoter is active in hiPS cells.

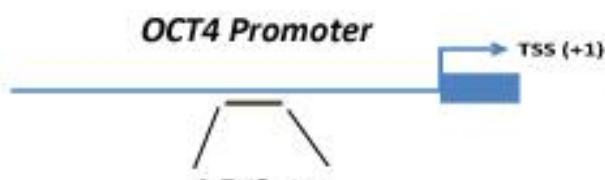

ㅊํำํํำำำ

hFB kas01

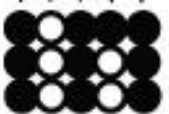

$66.7 \%$

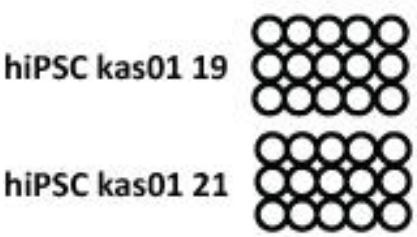

hFB Hess

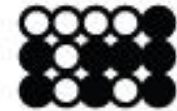

$53.3 \%$

$0 \%$

hiPSC kas02 23

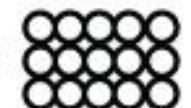

$0 \%$

$0 \%$

hiPSC kas02 14

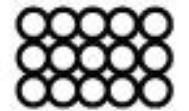

$0 \%$

hFB kas05

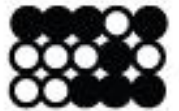

$53.3 \%$

hiPsc Hess 980808

$0 \%$

hiPSC kas05 05

80008

$0 \%$

hiPsc Hess 680808

$0 \%$

hiPSC kas05 29

$0 \%$

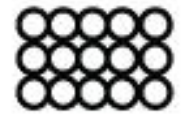

\section{Methylated O Unmethylated}

Figure 3.6: Methylation Status of OCT4 Promoter.

The CpG dinucleotides in the OCT4 promoter (-2126 -1891 bp from the TSS) were examined for the methylation status. Open circles represent unmethylated $\mathrm{CpG}$ dinucleotides; closed circles represent methylated CpG dinucleotides. The cell line is indicated to the left of each 
cluster. The percentage of all methylated CpGs for each promoter per cell line is noted to the right of each panel.

\section{in vitro and in vivo Differentiation}

The competence to differentiate into almost all cell types is the specific feature of human pluripotent stem cells (hPSCs). Embryoid bodies (EBs) formation is an in vitro test for PSCs. EB recapitulates many aspects of cell differentiation during early embryogenesis, and shows the ability of ES cells to differentiate into a variety of cell types in vitro. (Kurosawa, 2007) Another tool to monitor the capability of differentiation in stem cell research, also known as "gold standard" for assessing pluripotency, is teratoma formation in vivo. Teratomas are benign tumors composed of tissue or organ components from all three germ layers (endoderm, ectoderm, and mesoderm). When PSCs were injected into immune-deficient mice, PSCs develop into teratomas typically by remodeling their microenvironment to support their growth and generating blood vessels for nutrients (W. Y. Zhang, de Almeida, \& $\mathrm{Wu}, 2008)$.

To verify the ability of differentiation in vitro, the Control/PD-hiPSC floating culture was set up to form embryoid bodies (EBs) (Figure 3.7.I). After one week of suspension culture, the resulting cell aggregates (EBs) were plated on the gelatinized coverslips and kept growing in differentiation medium without bFGF growth factor. The outgrowth of differentiated cells from EBs presented different population of cell lineages: Endoderm ( $\alpha$-Foetoprotein), Mesoderm (Brachyury), and Ectoderm (Neuron-specific class III beta-tubulin) as documented by immunohistochemistry (Figure 3.7.II).

Transplantation of Control/PD-hiPSCs into immunodeficient mice conducted the test for teratoma formation in vivo. If the tumor formation were observed, tumors would collect for histological analysis. Histological structures representing endoderm, mesoderm and ectoderm lineages showed that the derived hiPSCs have pluripotent potential (Figure 3.7.III). 

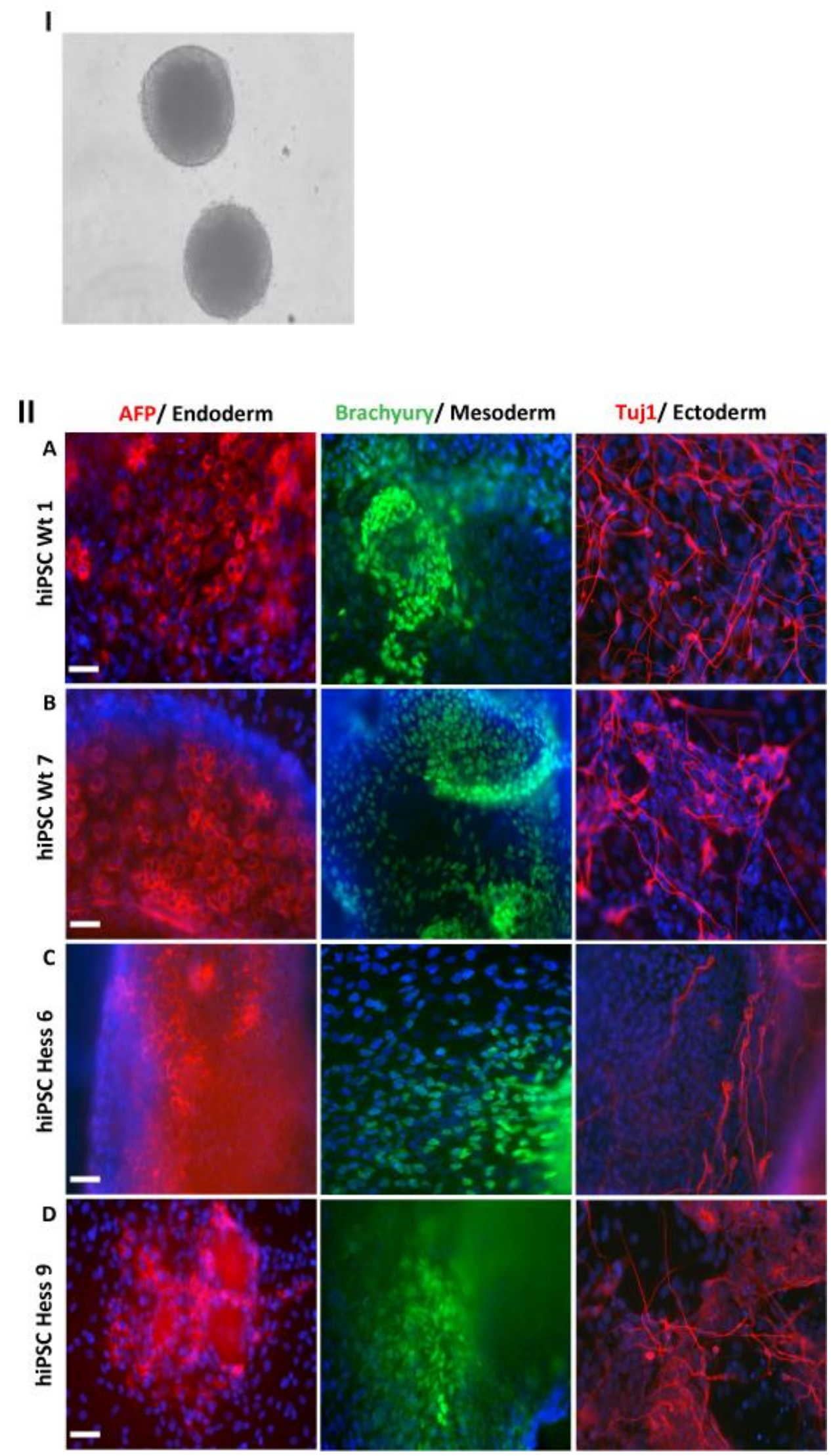


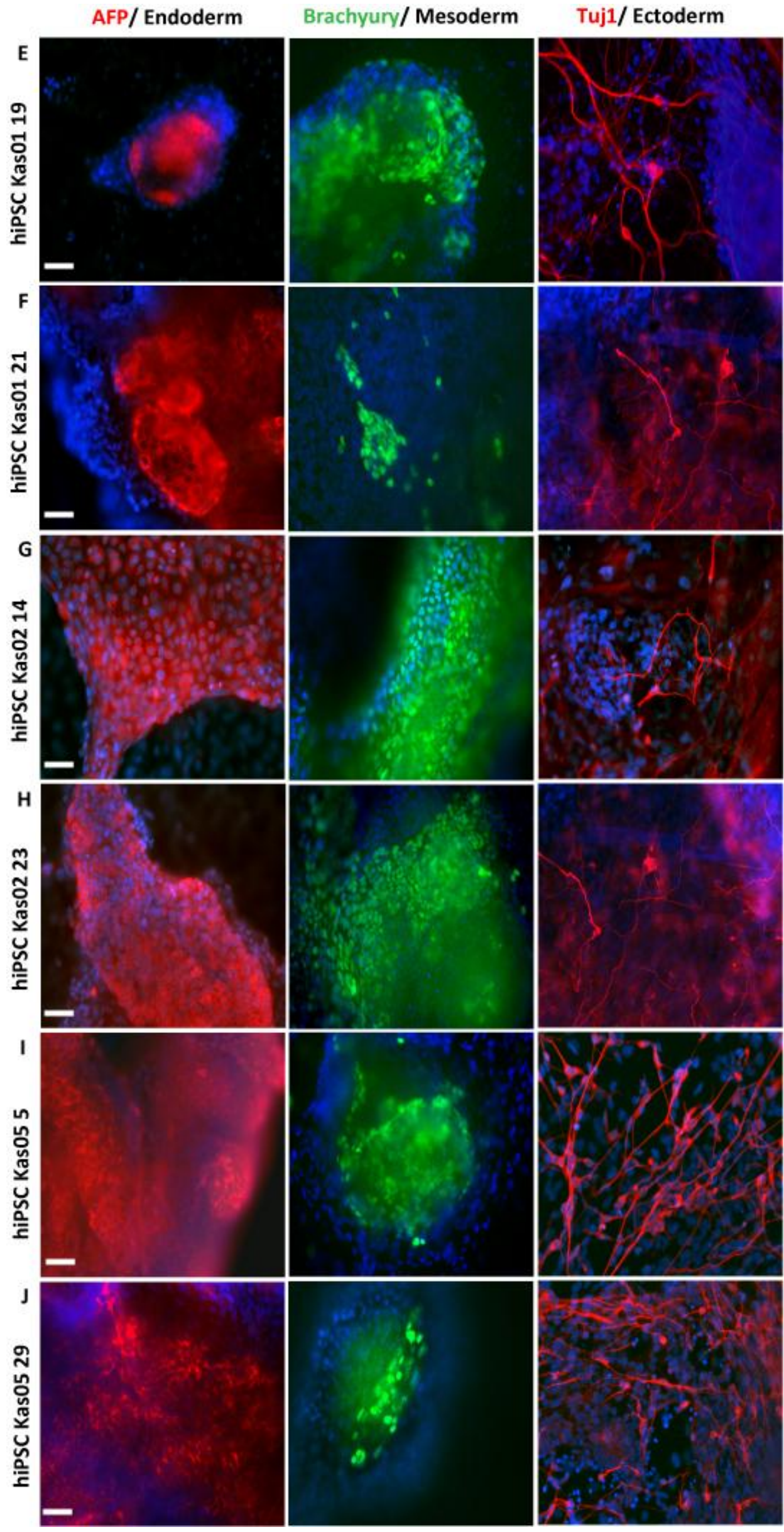




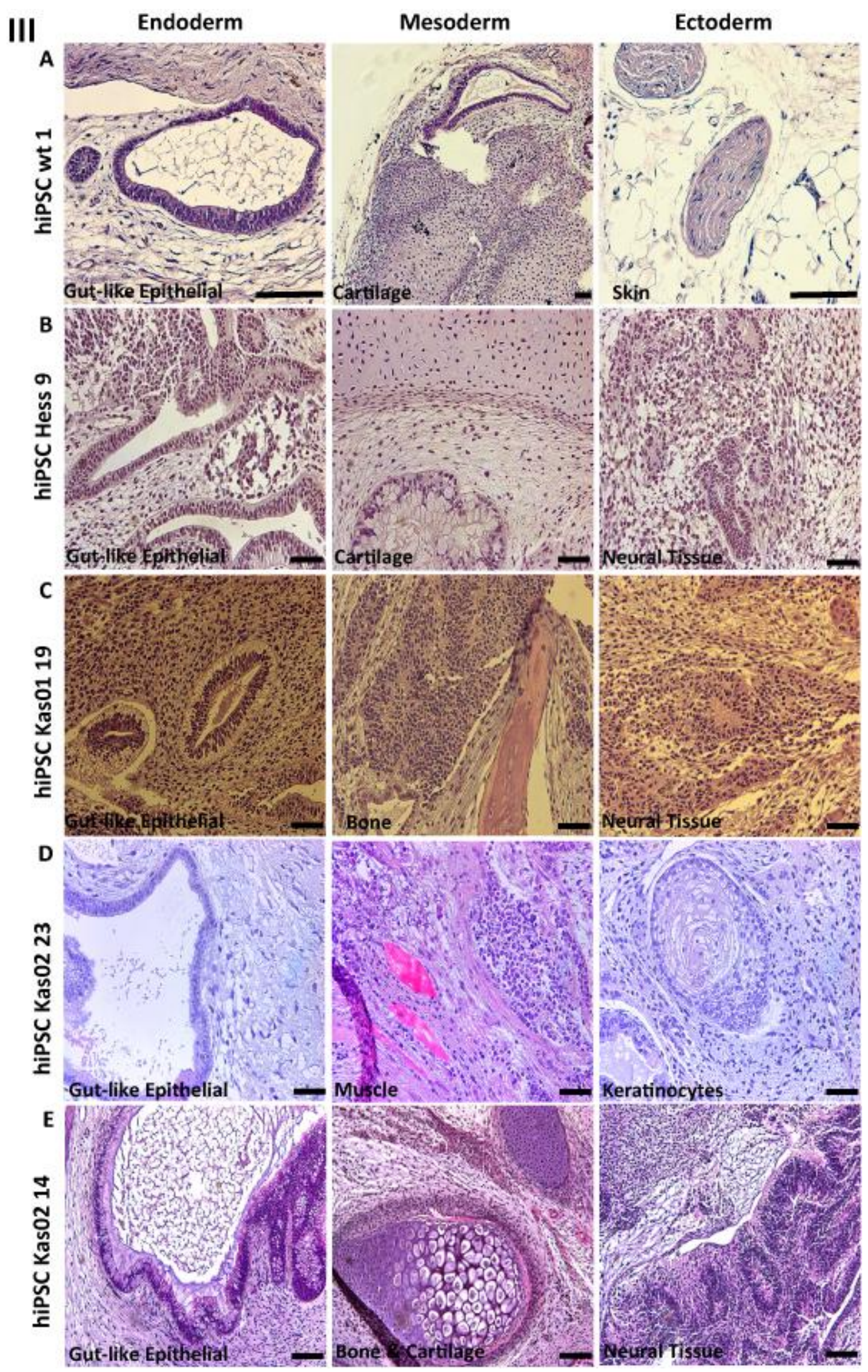




\section{Figure 3.7: in vitro and in vivo Differentiation of hiPSCs.}

(I) Embryoid body (EB) formation was carried in suspension culture of hiPSCs. (II) The outgrowth of adherent EBs derived from Control (wt 1 (A), 7 (B) \& Hess 6(C), 9 (D)) and PD (Kas01 19 (E), 21 (F) \& Kas02 14 (G), 23 (H) \& Kas05 5(I), 29 (J)) hiPSC were analyzed by immunostaining of different lineage markers. AFP ( $\alpha$-Foetoprotein): Endoderm; Brachyury: Mesoderm; Tuj1 (Neuronal Class III $\beta$-Tubulin): Ectoderm. White bar represents $50 \mu \mathrm{m}$. (III) hiPSC were injected into Immunodeficient mouse recipients. Resulting teratomas from control hiPSCs (wt 1 (A), Hess 9 (B)) and PD hiPSCs (Kas01 19 (C), Kas02 23 (D) \& Kas02 14 (E)) were histologically examined by HE staining. Gut-epithelial, cartilage \& muscle \& bone, skin \& keratinocyte \& neural tissue correspond to endo-, meso-, and ectodermal lineages, respectively. Black bar represents $50 \mu \mathrm{m}$. 


\subsection{Derivation of Neural Precursors from Control/PD-hiPSCs}

\subsubsection{Direct Differentiation of Neural Precursors form Control and PD hiPSCs}

Current developed protocols for neural induction of human pluripotent stem cells (hPSCs) rely on embryoid body (EB) formation (S. C. Zhang, et al., 2001) or stromal feeder co-culture (Kawasaki, et al., 2000). In addition, it is evident that inhibition of SMAD signaling improves the efficiency of neural induction. Therefore, some identified BMP inhibitors, like Noggin (Smith \& Harland, 1992) and Dorsomoprhin, have been used as neural inducing factors in several PSC neural differentiation protocols (Morizane, Doi, Kikuchi, Nishimura, \& Takahashi, 2011) (S. M. Chambers, et al., 2009). For neuron differentiation in hPSCs, it is often carried out on co-culture with stromal feeders with respect to the elicited stromal cell derived inducing activity (SDIA) observed in mouse ESC efficient neuronal differentiation. However, the effect is relatively less in human ESC, suggesting that additional treatments of BMP inhibitors accelerate and improve the neural induction. In this study, the combination of stromal feeder co-culture and BMP inhibitor treatment was set up to commit control/PD-hiPSCs to neuroectodermal lineage, generate multipotent neural precursors, and then further differentiate to dopaminergic neurons.

The day before starting neural induction, hiPSCs were clonal passaged onto inactive PA6 cells (stromal cell line derived from mouse skull bone marrow). Then the hiPSCs were co-cultured on PA6 feeders and treated with BMP inhibitors (either recombinant Noggin protein or small molecule Dorsomorphin). (Figure 3.8) After one week of neural induction, neural rosette structures (Figure 3.9.C) started to appear on the neural induced hiPSC colonies. By this time point, the expression of the neuroepithelial cell marker-Pax6 (Figure 3.9.A) as well as the neural stem/progenitor cell marker-Nestin (Figure 3.9.B) was present in neural induced cells. The neural rosettes were isolated mechanically and plated on poly-D-Lysine (PDL) coated dishes separately. Meanwhile, the neural rosettes were fed with Neural Precursor (NP) medium (serum free N2B27 defined medium) containing bFGF and EGF growth factors. In the presence of bFGF and EGF, hES-derived rosettes are able to proliferate and maintain the expressions of neural stem cell markers, Sox1, Sox2, and nestin (Elkabetz et al., 2008). After some days, the cell outgrowth of neural rosettes was observed. Following Accutase treatments, the neural rosettes were dissociated into single cells and replated onto new dishes. Those emerging neural precursors display the bipolar morphology maintaining the 
characteristics of neural rosette (Figure 3.9.D). The neural precursors grew as monolayers on the PDL coated dishes and were expansible with Accutase. When derived neural precursors were grown in suspension, they were able to form the neurospheres (Figure 3.9.E).

The generated transgene-free control/PD-hiPSCs were employed to derive multipotent neural precursors through this combination of BMP inhibitor treatments and co-culture on PA6 stromal feeders. Those control/PD-hiPSC derived neural precursors displayed the neural epithelial cell morphology and expressed neural precursor specific markers, e.g. Msi1, Sox1, Sox2, Nestin, and Pax6 (Figure 3.10). To date, control/PD-hiPSC derived neural precursors can be maintained up to passage 25 .

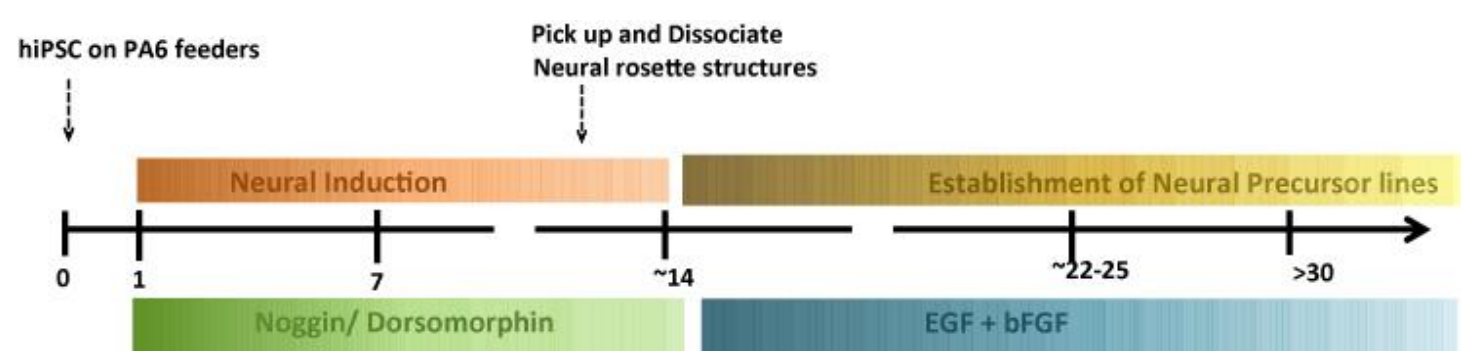

Figure 3.8: Flow Chart of Derivation of Neural Precursors. 

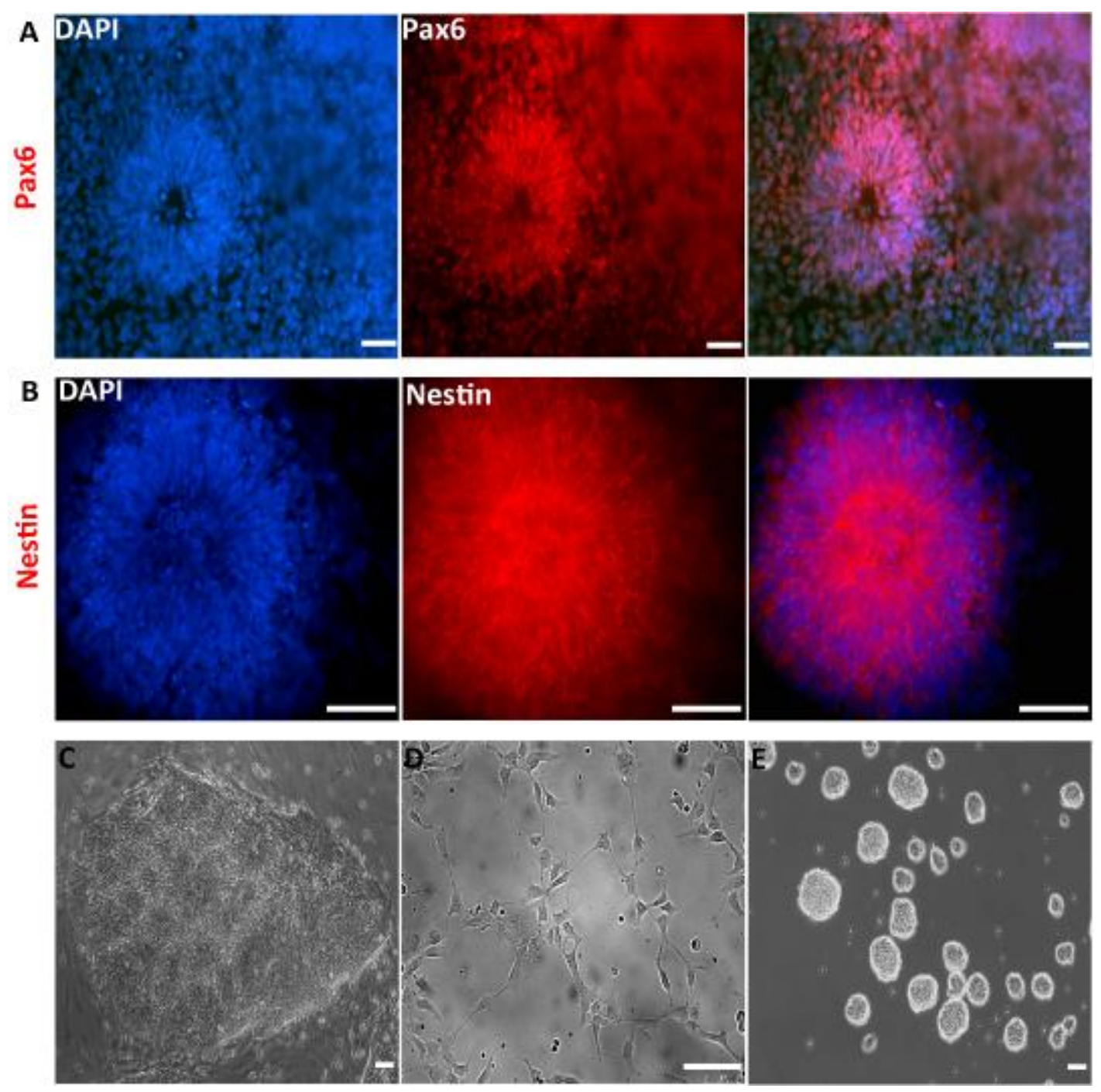

Figure 3.9: Neural Rosettes Structures in Neural Inductions.

Neural epithelial markers, Pax6 (A) \& nestin (B), were observed in neural rosettes during neural inductions. Nuclei were counterstained with DAPI (blue). The morphology of neural rosettes (C) was present in a neural induced hiPSC clone. Neural precursors displayed bipolar neural epithelial morphology (D). Neural precursors were able to form neurospheres (E). White scale bar corresponds to $100 \mu \mathrm{m}$. 


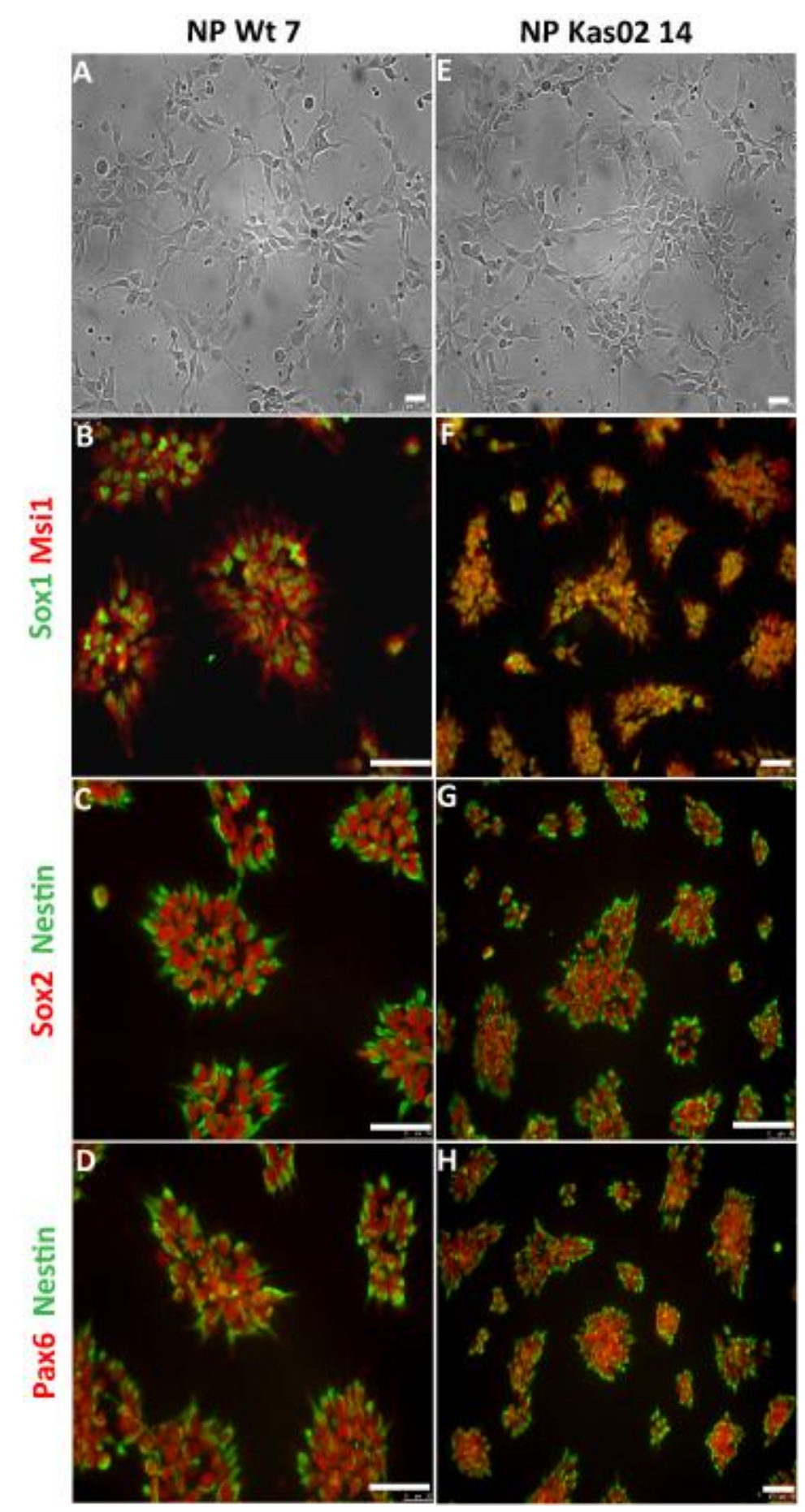

Figure 3.10: Derivation of Neural Precursors from Control/PD-hiPSCs Neural Inductions.

(A \& E) cell morphology of control \& PD hiPSCs derived neural precursors. These neural precursors expressed neural epithelial markers, Sox1 \& Msi1, Sox2, Pax6, and Nestin (B-D \& F-H). White scale bar corresponds to $50 \mu \mathrm{m}$ 


\subsubsection{Generation of dopaminergic neurons from Control/PD-hiPSC derived}

Neural Precursors

After the establishment of PD patient specific neural precursor cell lines from the pluripotent state, further differentiation was required to demonstrate the ability of conversion into terminally differentiated neurons. To enrich for dopaminergic neurons, PD relevant cell type, neural precursors were exposed to Shh and FGF8 growth factors to mediate the cell fates of dorsal-ventral patterning for two weeks. Afterwards, the differentiated cells were maintained in the neural differentiation medium containing BDNF, GDNF, dbcAMP, Ascorbic acid, and TGF- $\beta 3$ for maturation of dopaminergic neurons and to prevent cell death.

After 30 days of final dopaminergic maturation, control/PD-hiPSC derived neural precursors were able to differentiate into neuronal cells. They showed the expression of Neuronal Class III $\beta$-Tubulin (Tuj1), a pan neuronal marker (Figure 3.11). More importantly, the expression of Tyrosine hydroxylase (TH), the marker for dopaminergic neurons, was observed in differentiated neurons as well. 

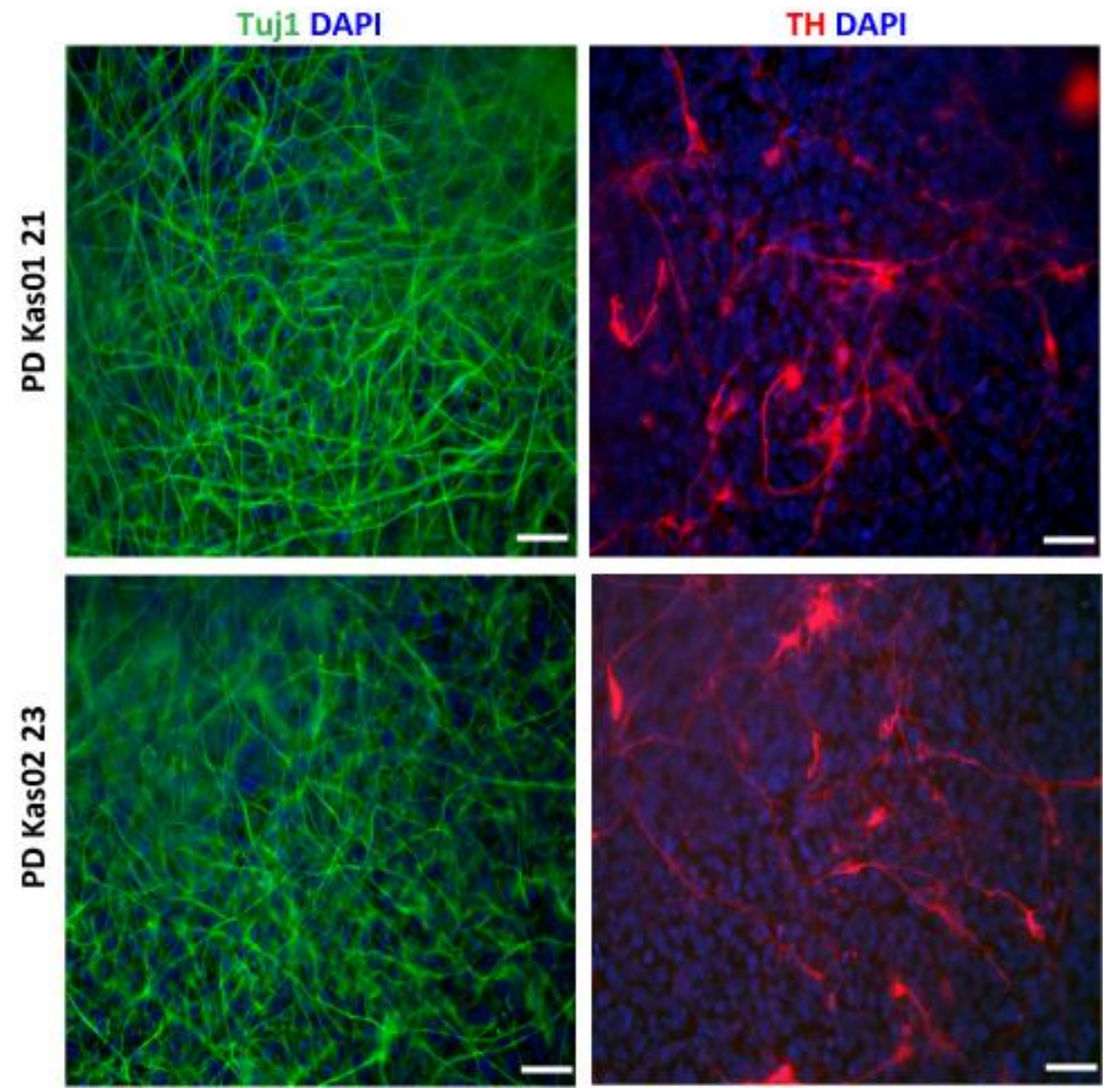

Figure 3.11: Dopamine Neuron Differentiation from PD-hiPSC derived Neural Precursors.

Immunofluorescent images of differentiated neurons following dopaminergic neuron patterning and maturations. Tuj1 (Neuronal Class III $\beta$-Tubulin) (green) represented as pan neuron marker while TH (Tyrosine Hydroxylase) (red) indicated dopamine neurons. Nuclei were counterstained with DAPI (blue). White scale bar corresponds to $100 \mu \mathrm{m}$. 


\subsubsection{Transcriptome Analysis of Control/PD-hiPSC Derived Neural Precursors}

On account of the increased knowledge of human genome, the technology of gene microarray has provided a rapid and parallel assay of gene expression patterns. The plenty of obtained data from the microarray platform can be used to assign a comprehensive understanding of genetic networks. Thus, it is a mighty technique to evaluate the global gene expression changes correlated with differential treatments of the samples and to demonstrate the correlation among various samples. Furthermore, it is possible to discover the new regulatory networks by the differential gene expression patterns.

To reveal novel molecular players involved in the PD pathogenesis, the control/PD-hiPSC derived neural precursors were used for transcriptome analysis using RNA microarray. 4 control neural precursors (wt 1, wt 7 \& Hess 6, Hess 9) were derived from 2 healthy persons' hiPSCs and 6 PD neural precursors (Kas01 19, Kas01 21 \& Kas02 14, Kas02 23 \& Kas05 5, Kas05 29) were derived from 3 individual PD patients' hiPSCs, respectively. The RNA microarray of control/PD-hiPSC derived neural precursors and initial data processing were carried out by the "Transkriptomanalyselabor (TAL)" at the University of Göttingen. The hierarchical correlation among the corresponding control and PD neural precursors was presented by the heatmap (Figure 3.12). The slight discrepancy between clones of the same sample can be observed, and might be due to the process of derivation of neural precursors. On account of individual samples with diverse genetic backgrounds, the global transcriptomes of respective samples' neural precursors were not similar to each other. Using the platform "GeneSifter ${ }^{\circledR ”}$ (PerkinElmer Inc.), the differential gene expressions of respective neural precursors were computed by Dr. Patrick Collombat, Institut de Biologie Valrose, Nice, France. To be considered differentially expressed between PD neural precursors versus control ones (PD-Control), genes had to be at least 1.5 folds up- or down-regulated and the corresponding $p$-value should be less than or equal to 0.05 . The number of resulting up- and down-regulated genes among PD-Control was 485 and 332, respectively.

To comprehensively understand the role of differentially expressed genes and their involved signaling pathways, total 817 genes (up- and down-regulated) were upload to the DAVID Bioinformatics Resources 6.7 (http://david.abcc.ncifcrf.gov/) for functional annotation and enrichment analyses. Based on the functional clustering, 13 pathways ( $p$-value less than or 
equal to 0.05 ) were depicted by KEGG pathways (Figure 3.13.A). Among them, the pathway of axon guidance was most significant and might be associated with PD (Lesnick et al., 2007) (J. M. Kim et al., 2011). The fold changes (PD versus Control) of 14 genes involved in axon guidance were presented on Figure 3.13.B and S1. Besides, the genes counted in Gene Ontology (GO) term for neuron differentiation (GO0030182) were picked up and displayed the fold changes (PD versus Control) in Table 3.2. Of note, EPHA4 gene was significantly increased ( 3.5 fold) in the pool of PD-hiPSC derived neural precursors and annotated in both of axon guidance and neuron differentiation. Besides, FGF20 was attributed to the scope of GO term: neuron differentiation (Table3.2) and remarkably reduced (4 fold) in PD-hiPSC derived neural precursors among genes of susceptibility to Parkinson disease (e.g. GAK (Cyclin G associated kinase), DGKQ (Diacylglycerol kinase, theta 110kDa), and FGF20 (Fibroblast Growth Factor 20)) (Pankratz et al., 2009) (Lill et al., 2012) (van der Walt et al., 2004 ) in this transcriptome analysis (Figure 3.14.A). Besides, the genetic variability of FGF20 has long been considered as a risk factor of PD (van der Walt, et al., 2004). Furthermore, the decreased expression level of FGF20 was verified among respective clones of PD-hiPSC derived neural precursors by QRT-PCR.

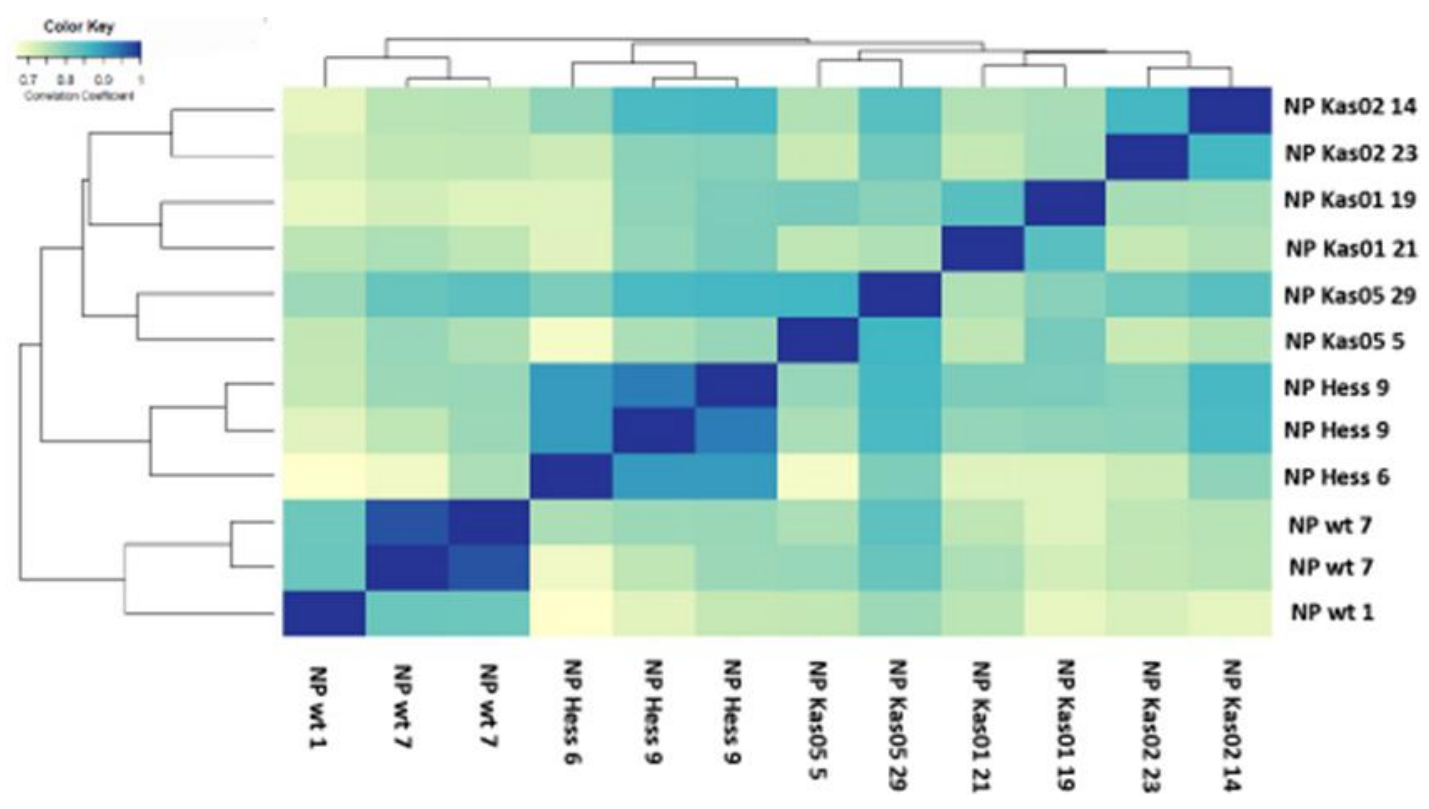

Figure 3.12: Comparative Transcriptome Analysis of Control/PD-hiPSC derived Neural Precursors.

This heatmap showed the correlation among controls (wt \& Hess) and PD (Kas01, Kas02 \& Kas05) samples. For each sample (Control and PD), 2 clones of neural precursors derived 
from 2 individual hiPSC clones were used as duplicate for analysis of RNA microarray. For the reason of fitting the experimental samples to the Aglient chip, NP wt 7 and NP Hess 9 were applied one more time.

A.

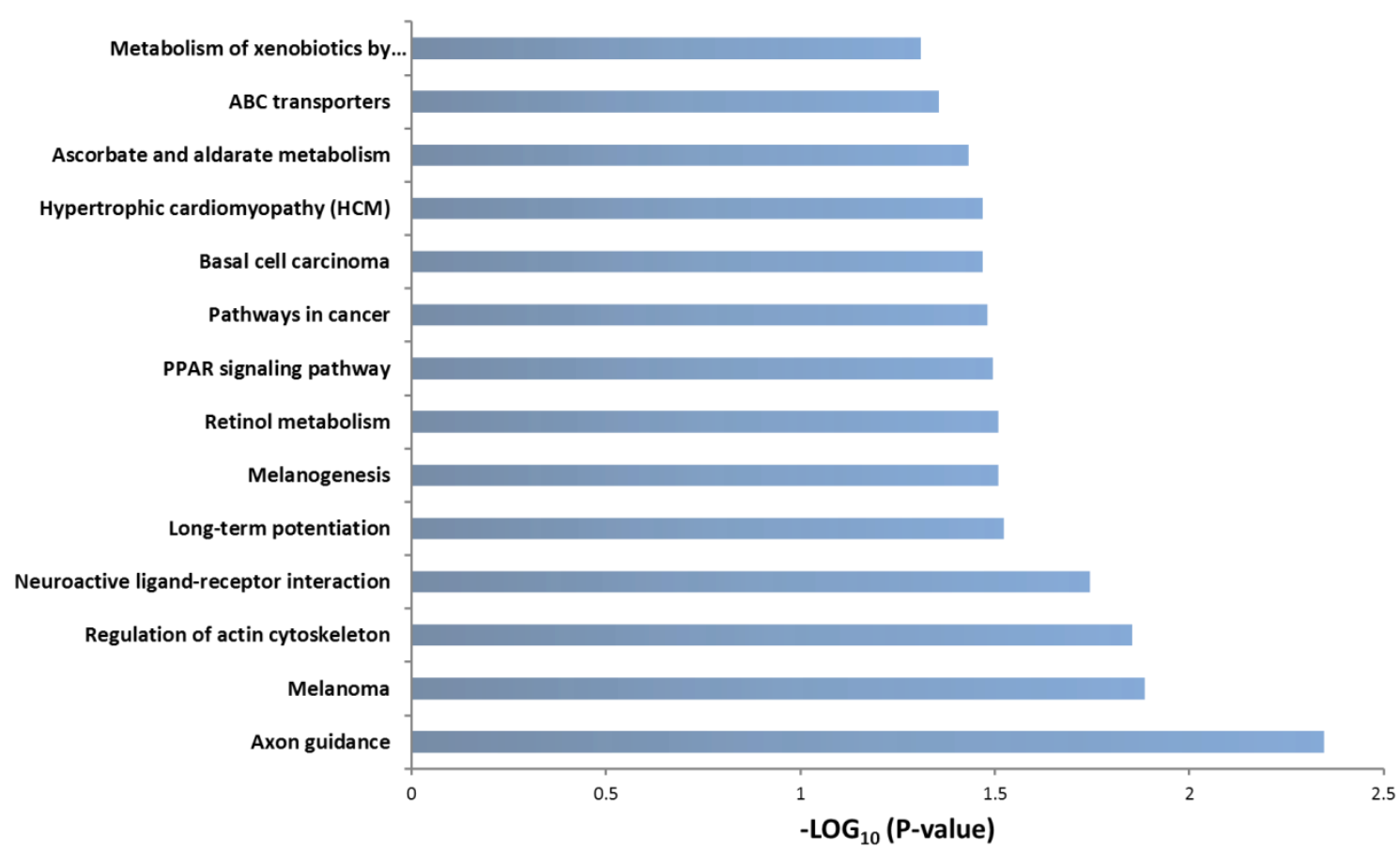

B.

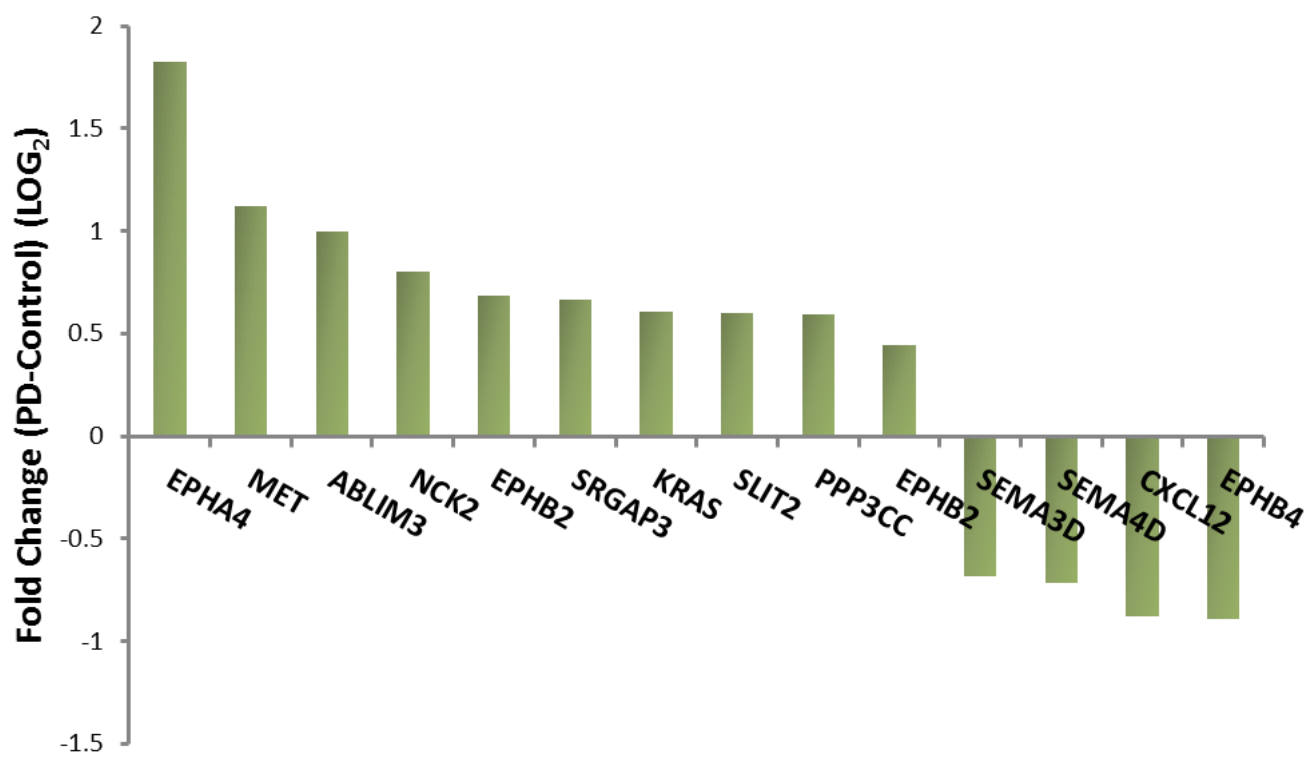

Figure 3.13: Functional Annotation Clustering by KEGG pathway. 
(A) showed the significance of enriched pathways clustered by total differential expressed genes. (B) presented the fold change (PD versus Control) of various genes in the pathway of axon guidance.

Table 3.2: Gene Ontology Analysis of Differential Gene Expression of Neuronal Precursors Annotated with the GO Term: Neuron Differentiation.

\begin{tabular}{|c|c|c|c|}
\hline REFSEQ_MRNA & $\begin{array}{l}\text { Fold Change } \\
\text { (PD-Control) } \\
\end{array}$ & $\begin{array}{c}\text { GENE } \\
\text { SYMBOL } \\
\end{array}$ & GENE NAME \\
\hline NM_001101 & 2.75 & ACTB & actin, beta \\
\hline NM_004438 & 1.82 & EPHA4 & EPH receptor $\mathrm{A} 4$ \\
\hline NM_000555 & 1.74 & DCX & doublecortin \\
\hline NM_001018065 & 1.67 & NTRK2 & neurotrophic tyrosine kinase, receptor, type 2 \\
\hline NM_004496 & 1.52 & FOXA1 & forkhead box A1 \\
\hline NM_201264 & 1.49 & NRP2 & neuropilin 2 \\
\hline NM_021955 & 1.49 & GNGT1 & $\begin{array}{l}\text { G protein, gamma transducing activity } \\
\text { polypeptide } 1\end{array}$ \\
\hline NM_004796 & 1.25 & NRXN3 & neurexin 3 \\
\hline NM_001145661 & 1.11 & GATA2 & GATA binding protein 2 \\
\hline NM_153487 & 1.04 & MDGA1 & $\begin{array}{l}\text { MAM domain containing } \\
\text { glycosylphosphatidylinositol anchor } 1\end{array}$ \\
\hline NM_002487 & 0.97 & NDN & necdin homolog (mouse) \\
\hline NM_005986 & 0.91 & Sox1 & SRY (sex determining region Y)-box 1 \\
\hline NM_000280 & 0.86 & PAX6 & paired box 6 \\
\hline NM_002167 & 0.86 & ID3 & inhibitor of DNA binding 3 \\
\hline NM_000052 & 0.82 & ATP7A & ATPase, $\mathrm{Cu}++$ transporting, alpha polypeptide \\
\hline NM_171999 & 0.80 & SALL3 & sal-like 3 (Drosophila) \\
\hline NM_005964 & 0.77 & MYH10 & myosin, heavy chain 10 , non-muscle \\
\hline NM_022363 & 0.69 & LHX5 & LIM homeobox 5 \\
\hline NM_000675 & 0.65 & ADORA2A & adenosine $\mathrm{A} 2 \mathrm{a}$ receptor \\
\hline NM_001719 & 0.62 & BMP7 & bone morphogenetic protein 7 \\
\hline NM_004787 & 0.60 & SLIT2 & slit homolog 2 (Drosophila) \\
\hline NM_004442 & 0.45 & EPHB2 & $\mathrm{EPH}$ receptor $\mathrm{B} 2$ \\
\hline NM_001982 & -0.66 & ERBB3 & $\begin{array}{l}\text { v-erb-b2 erythroblastic leukemia viral } \\
\text { oncogene homolog } 3 \text { (avian) }\end{array}$ \\
\hline NM_001033886 & -0.88 & CXCL12 & chemokine ( $\mathrm{C}-\mathrm{X}-\mathrm{C}$ motif) ligand 12 \\
\hline NM_032109 & -0.92 & OTP & orthopedia homeobox \\
\hline NM_002507 & -0.99 & NGFR & nerve growth factor receptor \\
\hline NM_014624 & -1.20 & S100A6 & S100 calcium binding protein $A 6$ \\
\hline NM_000230 & -1.40 & LEP & leptin \\
\hline NM_005172 & -1.60 & ATOH1 & atonal homolog 1 (Drosophila) \\
\hline NM_001426 & -1.92 & EN1 & engrailed homeobox 1 \\
\hline NM_019851 & -2.01 & FGF20 & fibroblast growth factor 20 \\
\hline
\end{tabular}

Note: Fold Change (PD-Control) $\mathrm{LOG}_{2}$ 
A.

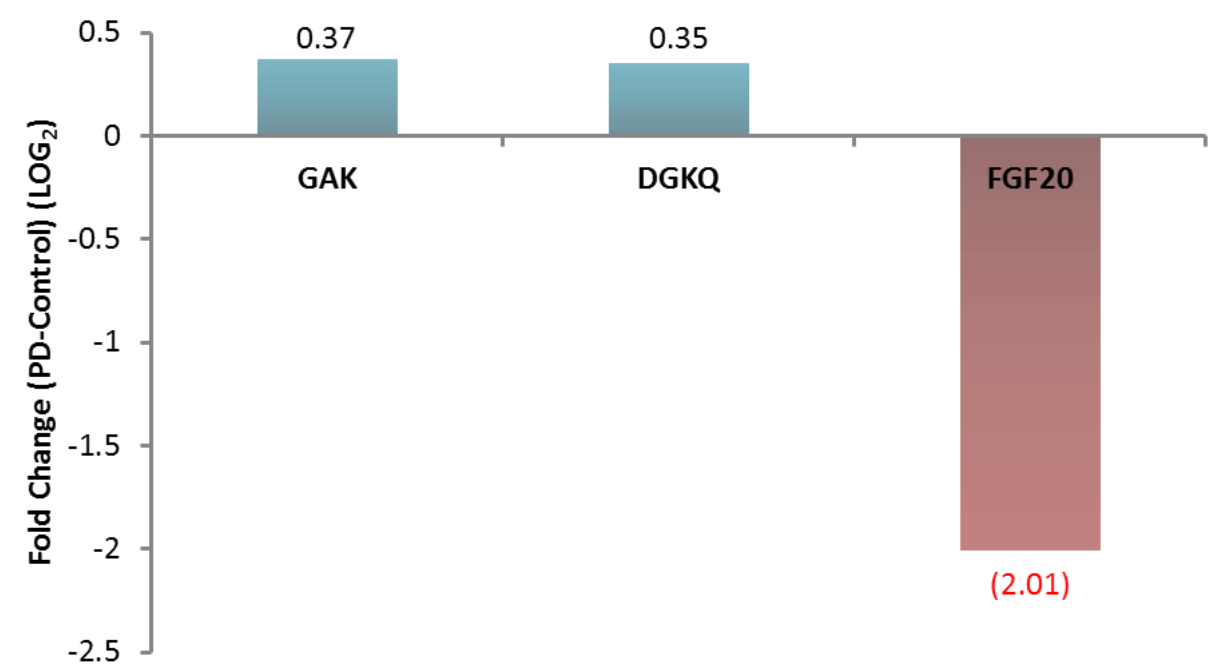

B.

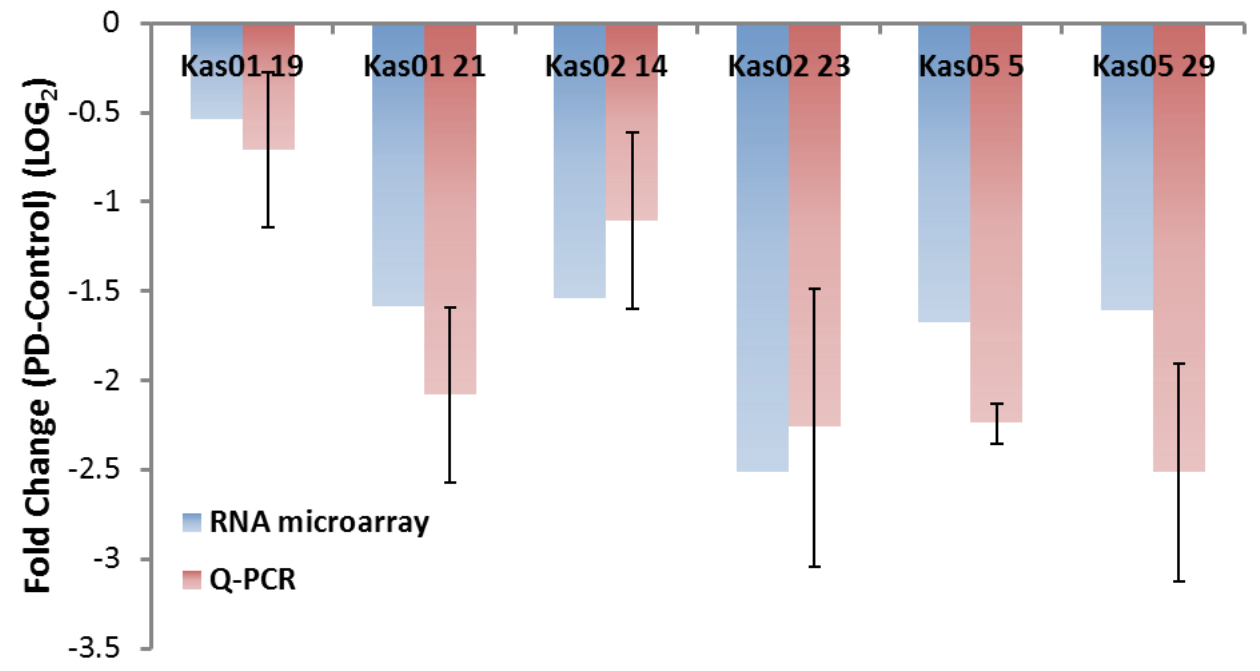

Figure 3.14: Expression Level of Genes of Susceptibility to Parkinson Disease.

(A) In the transcriptome analysis of control/PD-hiPSC derived neural precursors, 3 genes (GAK, DGFQ, and FGF20) associated with the susceptibility to PD were differential expressed. (B) showed the expression changes of FGF20 (PD-Control) for each clone of PD-hiPSC derived neural precursors in both of RNA microarray and Q-PCR. 


\subsection{Derivation of Floor Plated-based Differentiated mDA Neurons from Conrtol/PD-hiPSCs}

\subsubsection{Direct Differentiation of midbrain Dopaminergic Neurons from}

Control/PD-hiPSCs

In order to increase the number of midbrain dopaminergic neurons from control/PD-hiPSCs for functional and phenotype assays, the alternative strategy of differentiation, floor plate (FP)-based dopamine neuron differentiation, was conducted in this study. Since 2012, several protocols have been adopted for hPSCs, including hPSC monolayer culture, EB suspension culture, or hPSC co-culture with feeders (Kriks, et al., 2011) (Xi, et al., 2012) (Denham, et al., 2012). The general features are early activation of Shh and wnt signaling during neural induction of hPSC by inhibition of dual SMAD signaling. The essential transcription factor, also known as the marker of FP, FoxA2 would mark FP-based induced cells due to the activation of Shh. Through the induction of wht signaling, Lmx1a would be co-expressed on FoxA2 positive cells, then midbrain FP DA precursors emerged. Midbrain DA Neurons would appear to follow further differentiation and maturation of midbrain FP DA progenitors.

In this study, the differentiation of FP-based dopamine neurons was adopted and modified from (Xi, et al., 2012). (Figure 3.14) One day before differentiation, hiPSCs were clonal passaged onto inactive PA6 feeder layers. The next day, hiPSCs were fed with FP induction medium in the presence of SB431542 (SB) and LDN193189 (LDN) for neural induction. In the meantime, purmorphamine (pur) (an activator of Shh) and CHIR99021 (CHIR) (an activator of wnt) were added to the hiPSC to drive Shh-FoxA2 and Wnt1-Lmx1a regulatory loops. After 9 days of neural induction, dual SMAD inhibitors were removed, but the cells were still exposed to pur and CHIR for continuous activation of Shh and wnt signaling till day 13. At this time, the induced hiPSCs were prepared to examine the co-expression of FoxA2 and Lmx1a, the markers of midbrain FP precursors (Figure 3.15.A). Then the cells were fed with neuronal differentiated medium supplemented with a set of neurotrophic factors (BDNF, GDNF), growth factor (TGF-B3), and small molecules (Ascorbic acid, CAMP, and DAPT) for preventing cell death and promoting maturation of midbrain DA neurons. After 30 days of differentiation, the expressions of Tuj1 and TH were observed in a large proportion of cells 
(Figure 3.15.B). More importantly, the specific markers of midbrain DA neurons, Lmx1a and engrailed 1 (EN1), were present in TH positive cell as well (Figure 3.15.C \& D).

\subsubsection{Electrophysiological Analysis of FP-based Control/PD-hiPSC Derived mDA Neurons}

Neurons are cells specialized to processes and transmit information through electrical and chemical events (neurotransmitters). It is believed that neurons communicate with each other as well as with other terminal tissues through electrical activity. Therefore, the electrophysiological analysis of neurons is capable of showing hugh discernment into the function of neuronal elements.

hiPSC derived mDA neurons were used for the technique of whole cell patch clamp after 40 days of differentiation. From the result, it appeared that the differentiated cells (Figure 3.16.A) exhibited characteristics of neurons: Action potentials were triggered by injection of current (Figure 3.16.B); both inward $\mathrm{Na}+$ and outward $\mathrm{K}+$ currents were observed in these cells by voltage clamp (Figure 3.16.C \& D); the spontaneous excitatory postsynaptic current (ESPC) was observed (Figure 3.16.E). The results from the electrophysiological recordings demonstrated that the hiPSC derived mDA neurons presented neuronal functional properties, and were capable of synaptic transmission.

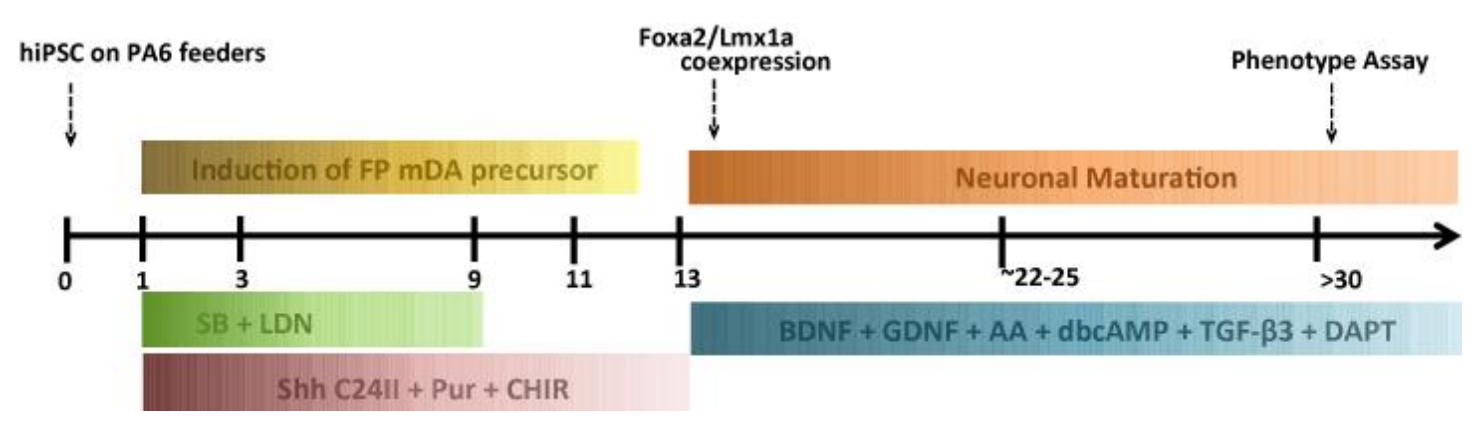

Figure 3.15: Flow Chart of FP-based Differentiation of Midbrain Dopaminergic Neurons. 

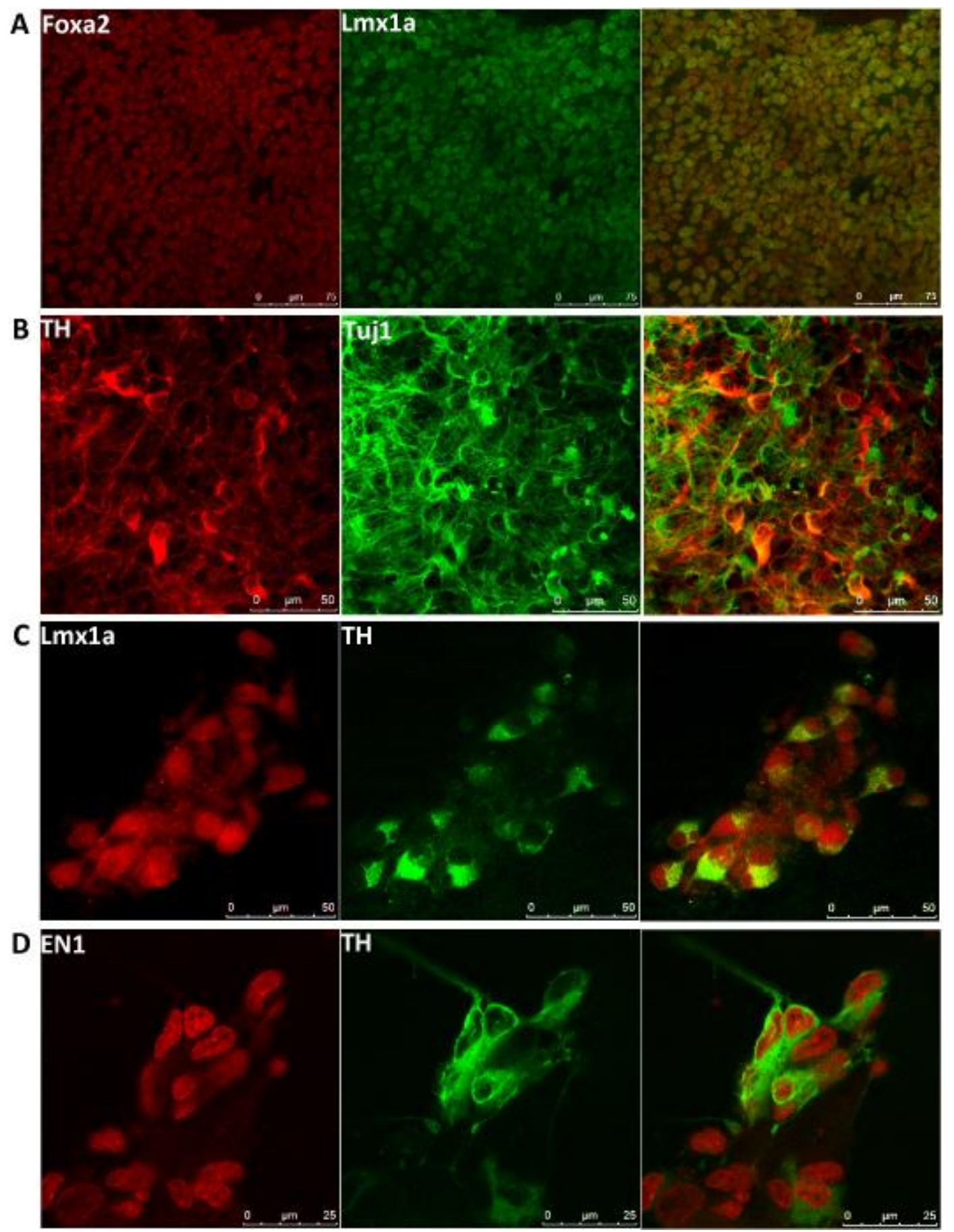

Figure 3.16: Induction and Maturation of hiPSC derived mDA neurons.

(A) Immunostaining showed colocalization of Foxa2 and Lmx1a in induced hiPSC at day13. (B) After one month of differentiation, $\mathrm{Tuj}^{+} / \mathrm{TH}^{+}$cell populations were observed. (C) $\mathrm{Lmx} 1 \mathrm{a}$ and (D) EN1, mDA neuron specific markers, were coexpressed in TH positive cells. 
A

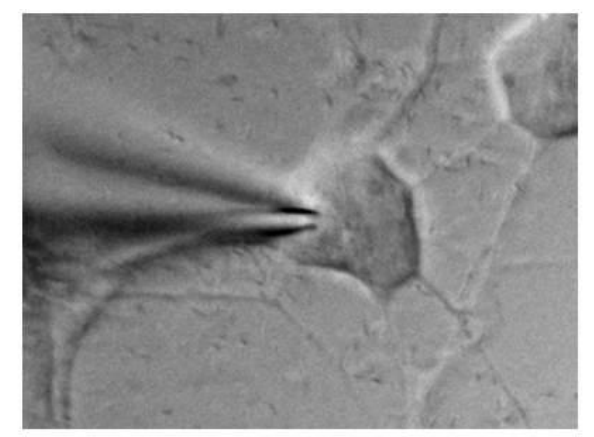

C

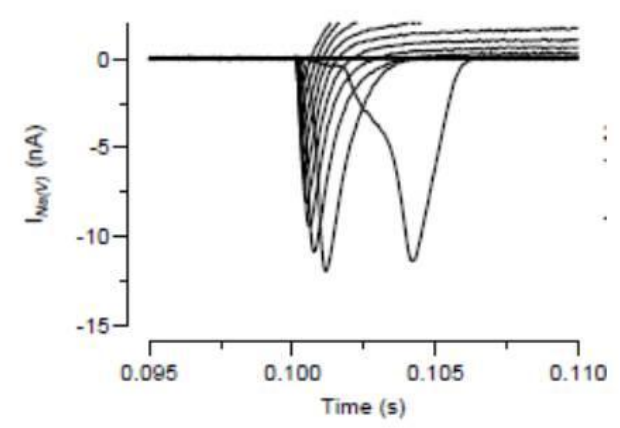

B

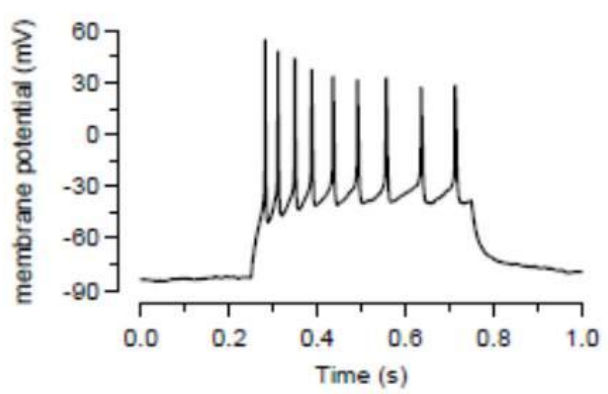

D

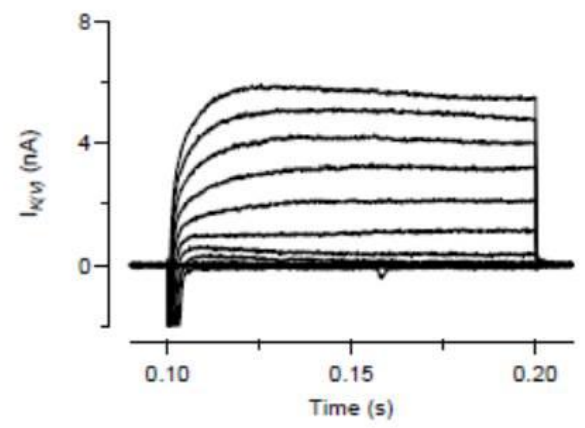

E
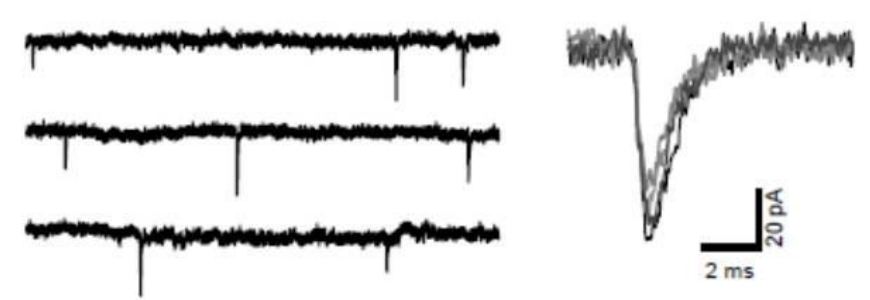

Figure 3.17: Electrophysiological Analyses of hiPSC Derived mDA Neurons.

(A) Phase contrast image of the patched cell with typical neuronal morphology was taken under the 60x water-immersion objective. (B) Action Potential (AP) elicited by a sustained (500 ms, $75 \mathrm{pA}$ ) current injection recorded in the current-clamp mode. (C) $I_{\mathrm{Na}(\mathrm{V})}$ and (D) $I_{\mathrm{Ka}(\mathrm{V})}$ were evoked by voltage step pulses $(50 \mathrm{~ms}$ and $100 \mathrm{~ms}$ ) from a holding potential of $-70 \mathrm{mV}$, starting at $-70 \mathrm{mV}$ with $10 \mathrm{mV}$ increments up to $+60 \mathrm{mV}$. Left is the representative traces and right is its I-V curve. (E) Baseline recording shows individual miniature EPSCS (mEPSCS) from hiPSC-derived cells. These spontaneously occurring events have amplitudes of $\sim 60 \mathrm{pA}$. 


\subsubsection{PD-hiPSC Derived mDA Neurons were Sensitive to CCCP-induced Mitochondrial Damage}

Parkinson's disease (PD) has long been proposed to be probably induced by the interaction of genetic predisposition and environmental factors. Some genetic defects (e.g. PINK1, Parkin \& LRRK2) associated with familial PD have linked to mitochondrial deficits (J. Y. Lee, Nagano, Taylor, Lim, \& Yao, 2010) (Matsuda, et al., 2010) (Nguyen et al., 2011). In particular, mitochondrial dysfunction and oxidative damage have been implicated in PD pathogenesis. Therefore, it is critical to examine the effects of mitochondrial dysfunction in PD-hiPSC derived DA neurons.

To evaluate the impact of mitochondrial malfunction in mDA neurons derived from hiPSCs, exogenous stress (e.g. mitochondrial toxin) was applied for phenotypic assay. After 30 days of FP-based dopamine neuron differentiation, the control/PD-hiPSC derived mDA neurons were treated with a high concentration of carbonyl cyanide m-chlorophenylhydrazone (CCCP) for $1 \mathrm{~h}$ to dissipate mitochondrial membrane potential (MMP) resulting in mitochondrial damage and cell death. It was followed by the immunohistochemical staining for TOM20, translocase of outer mitochondrial membrane, which indicated the localization of mitochondria in the TH positive DA neurons (Figure 3.17.A). Upon CCCP treatment fewer cells were alive in PD-hiPSC derived mDA neurons. Moreover, the expression of TOM20 was found to be reduced by the quantitative analysis of integrated density of TOM20 in PD-hiPSC derived mDA neurons (Kas01 19, Kas02 23) (Figure 3.4. C, D \& E) indicating that the mitochondrial network was disturbed. In comparison to mDA neurons derived from healthy control, PD-hiPSC derived mDA neurons showed stronger mitochondrial damage which might be caused by an enhanced sensitivity towards CCCP. 


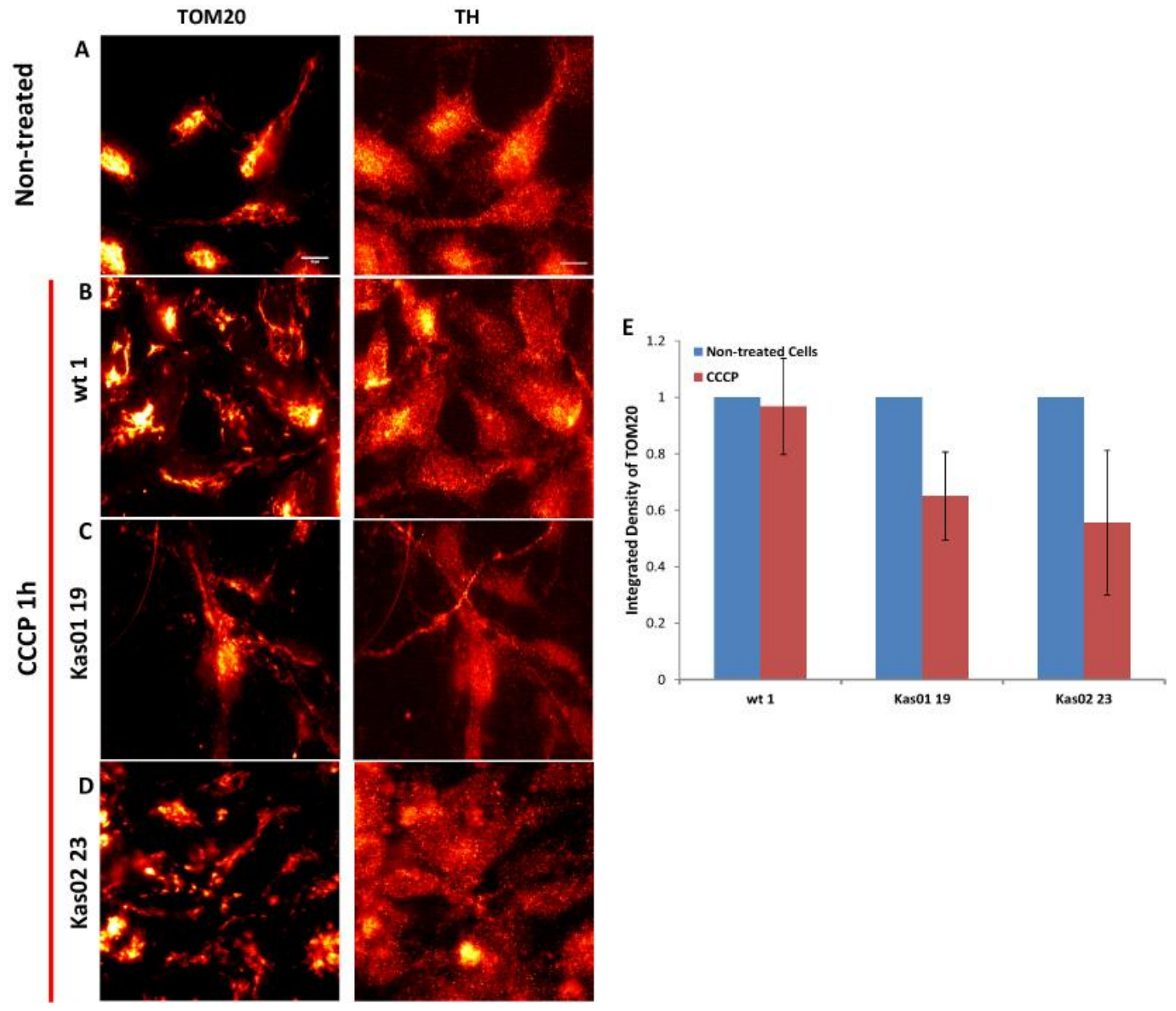

Figure 3.18: CCCP induced Mitochondrial Damage in hiPSC derived mDA Neurons.

After 30 days of FP-based mDA neuron differentiation, control/PD-hiPSC derived mDA neurons were treated with $100 \mu \mathrm{M} \mathrm{CCCP}$ for $1 \mathrm{~h}$ at $37^{\circ} \mathrm{C}$. Immunostaining of TOM20 and TH were used to mark the the mitochondrial outer membrane protein and the DA neurons. (A) Non-treated Cells, (B) control (wt), and (C) (D) PD (Kas01 \& Kas02) mDA neurons, respectively. White scale bar corresponds to $10 \mu \mathrm{m}$. Image acquisition was performed by Dr. Susann Kummer. (E) Quantification of integrated density TOM20 in mDA neurons. 2-5 cells per image were chosen to quantify the average integrated density of TOM20. Bars indicate the mean of two independent images \pm the standard error of the mean (SEM). 


\subsubsection{FGF20 Might Counteract the Mitochondrial Damage Induced by CCCP}

From the current results of transcriptome analysis, the expression of FGF20 was significantly down-regulated in PD-hiPSC derived neural precursors (Figure 3.13). FGF20 is part of the fibroblast growth factor (FGF) family with diverse functions in development and metabolism. In the brain, FGF20 is preferentially expressed in the region of substantia Nigra pars compacta (SNpc) (Ohmachi et al., 2000), and has been suggested to boost the development of dopaminergic neurons (Shimada, Yoshimura, Tsuji, \& Kunisada, 2009). Besides, FGF20 has been shown to protect mouse dopaminergic neurons against toxins in vitro and to restore some degree of motor function in a 6-OHDA-lesioned rodent PD model (Sleeman, Boshoff, \& Duty, 2012). Thus, FGF20 might elicit the restorative effect to counteract neurodegeneration of PD.

In order to examine the neuroprotective effect of FGF20 on PD-hiPSC derived mDA neurons, FGF20 was added to the differentiated control/PD-hiPSC derived mDA neurons 1 day prior to CCCP treatment. Consistent with the previous results, CCCP treated PD-hiPSC derived mDA neurons showed a disruption of the mitochondrial network (Figure 3.18.E, H \& K) that denoted mitochondrial damage due to uncoupling of the proton gradient and thus, loss of membrane potential resulting in the breakdown of the respiratory chain. Of note, if the cells were pretreated with FGF20 $(100 \mathrm{ng} / \mathrm{ml})$ for $24 \mathrm{~h}$, mitochondrial damage was reduced, which indirectly demonstrated the neuroprotective effect of FGF20 on hiPSC derived PD mDA neurons (Figure 3.18.F, I \& L). 


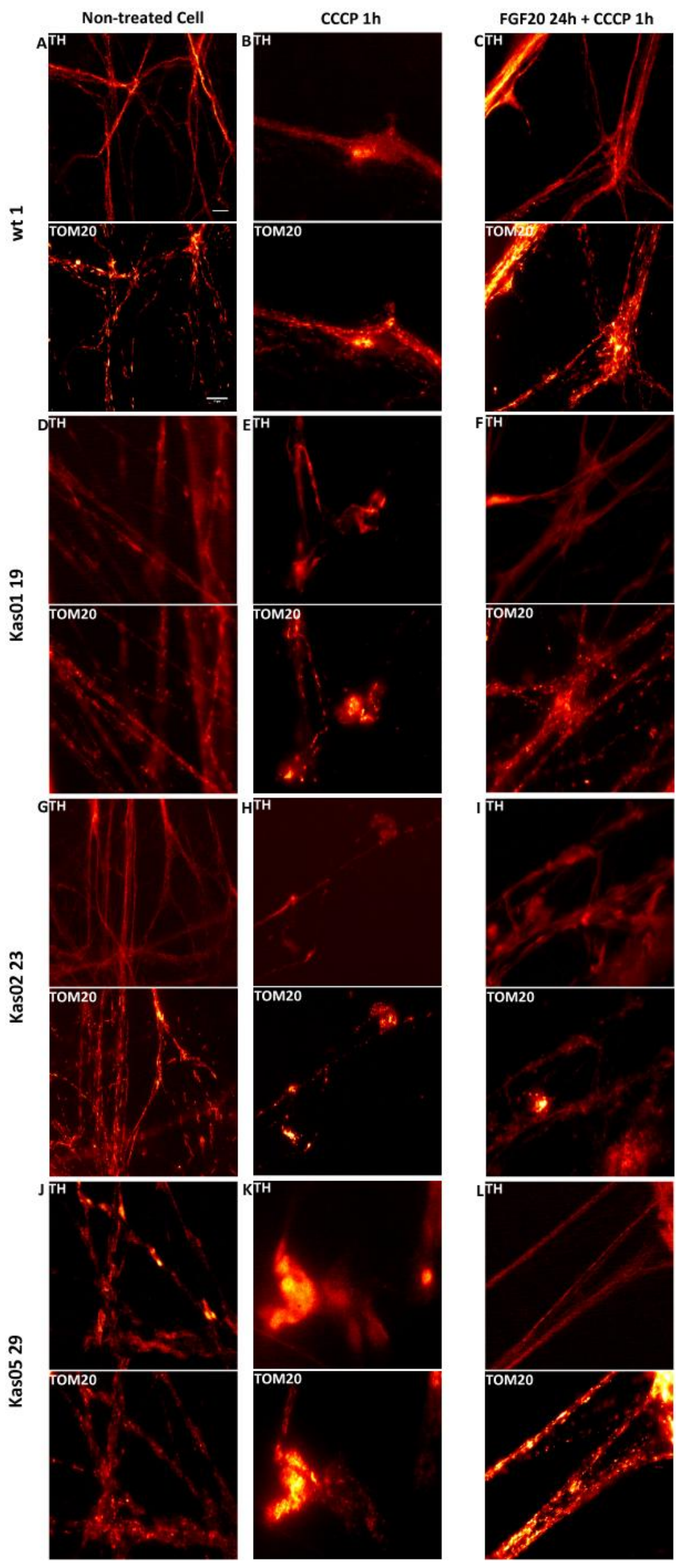


Figure 3.19: Effect of FGF20 on CCCP Triggered Mitochondrial Damage in hiPSC-derived mDA Neurons.

$100 \mathrm{ng} / \mathrm{ml}$ FGF20 was added to the control/PD-hiPSC derived mDA neurons one day before $100 \mu \mathrm{M}$ CCCP treatment. Immunostaining of TOM20 and TH marked the mitochondrial outer membrane protein and the DA neurons, respectively. (A), (D), (G), (J) non-treated cells, (B), $(E),(H),(K)$ CCCP treated cells, (C), (F), (I), (L) cells pretreated with FGF20 followed by CCCP treatment. $(A) \sim(C),(D) \sim(F)$, and $(G) \sim(I)$ presented control (wt) and PD (Kas01, Kas02 \& Kas05) hiPSC derived mDA neurons, respectively. Image acquisition was performed by Dr. Susann Kummer.

\subsubsection{FGF20 Might Reduce the Excess Intracellular Reactive Oxygen Species (ROS)}

To examine the neuroprotective effect of FGF20 on oxidative damage in PD patient hiPSC derived mDA neurons, $\mathrm{H}_{2} \mathrm{O}_{2}$-mediated oxidative stress was employed. FGF20 was added to the differentiated control/PD-hiPSC derived mDA neurons 1 day before the cells were exposed to a high concentration of $\mathrm{H}_{2} \mathrm{O}_{2}$. Afterwards, the $\mathrm{H}_{2} \mathrm{O}_{2}$ remaining in the medium was removed and the cells were incubated with the cell-permeable 2', 7'-dichlorodihydrofluorescein diacetate (H2-DCFDA), a general oxidative stress indicator for detection of intracellular ROS. Upon the cleavage by the intracellular oxidation, the non-fluorescent H2-DCFDA is converted to fluorescent DCF. Using flow cytometer, the fluorescence of living cells was monitored to correspond to the amount of intracellular ROS (Figure 3.19.A). High concentration of $\mathrm{H}_{2} \mathrm{O}_{2}$ induced an acute increase in ROS within the control/PD-hiPSC derived mDA neurons. It was noted that pretreatments of FGF20 could reduce some degree of excess intracellular ROS. Although the results were not significant, the tendency of decrease of ROS level upon FGF20 pretreatments could be observed (Figure 3.19.B). Similar to the effect seen in CCCP treatments, it suggested that FGF20 indirectly counteracted the dysfunction of mDA neurons caused by either mitochondrial damage or oxidative stress. 
A.

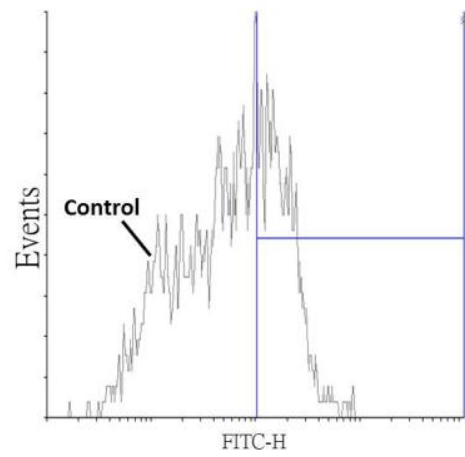

Non-treated Cells

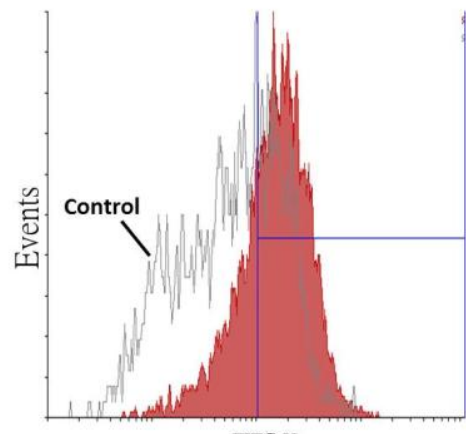

$\mathrm{H}_{2} \mathrm{O}_{2} \mathrm{Ih}$

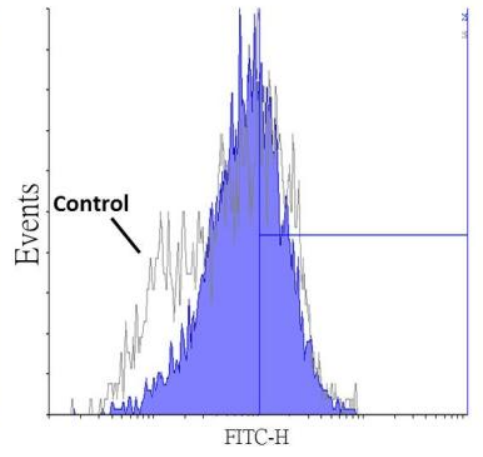

FGF20 $24 \mathrm{~h}+\mathrm{H}_{2} \mathrm{O}_{2} 1 \mathrm{~h}$

B.

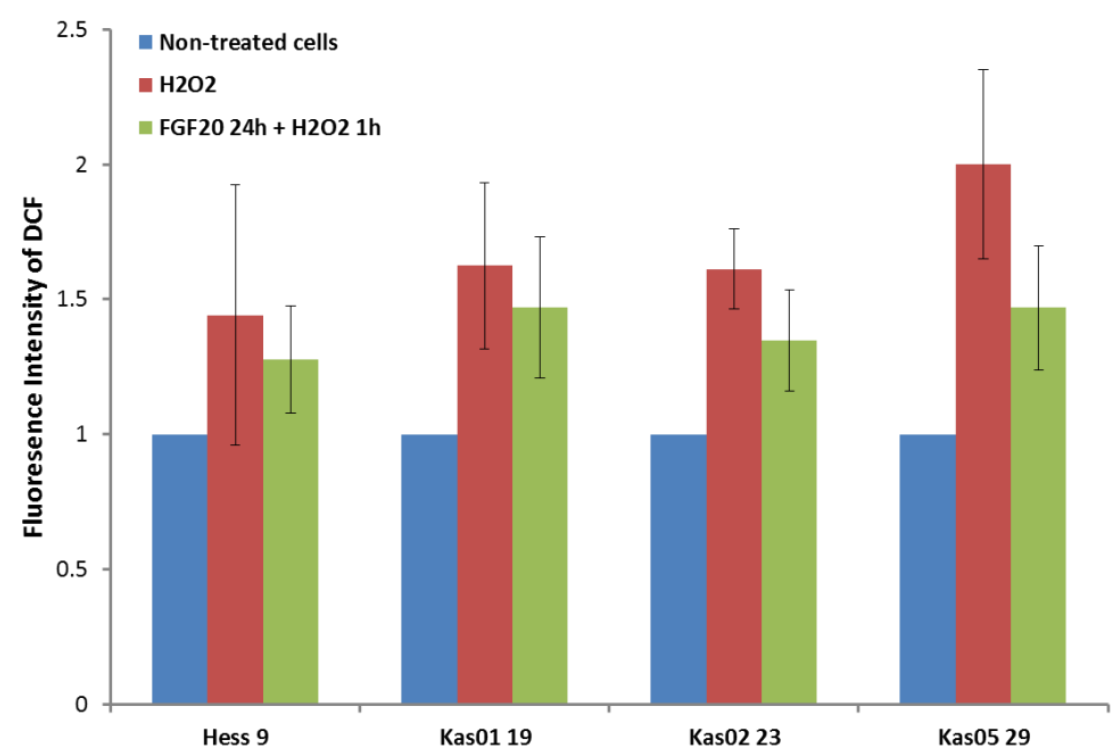

Figure 3.20: Effects of FGF2O on $\mathrm{H}_{2} \mathrm{O}_{2}$-induced Generation of Reactive Oxygen Species (ROS) in hiPSC-derived mDA Neurons.

The cells were preteated with FGF20 $100 \mathrm{ng} / \mathrm{ml}$ for $24 \mathrm{~h}$ followed by treatment with $100 \mu \mathrm{M}$ $\mathrm{H}_{2} \mathrm{O}_{2}$. The ROS production was measured by the fluorescence of DCF using flow cytometry as described in Methods. (A) The representative plots of the analyses (B) Quantitative analysis of ROS production. Bars indicate the mean of three independent experiments \pm the standard error of the mean (SEM). 


\section{Discussion}

In this study, 3 individual PD patients' dermal fibroblasts, together with 2 healthy persons, were converted to induced pluripotent stem cells (hiPSC) using a lentiviral polycistronic vector (hSTEMCCA). After conducting a series of tests to confirm the pluripotency, control/PD-hiPSCs were used to derive neural precursors and midbrain dopaminergic neurons with the purpose of investigation of PD pathogenesis. In an attempt to molecularly characterize iPSC that were generated from PD patients, we examined iPSC derived neural precursors using transcriptome analysis. From the result of transcriptome analysis of control/PD-hiPSC derived neural precursors, it is suggested that axon guidance pathway might be implicated in PD etiology and/or progression. Besides, the expression of FGF20, the gene associated with the risk of PD, was significantly reduced in PD-hiPSC derived neural precursors. Based on the phenotypic assay of control/PD-hiPSC derived mDA neurons, PD-hiPSC derived mDA neurons were more prone to the CCCP-induced mitochondrial damage. When the cells were exposed to FGF2O prior to external stress (e.g. CCCP or H2O2) treatments, the cellular damage was attenuated, suggesting that FGF20 elicit the neuroprotective effects on PD-hiPSC derived mDA neurons.

\subsection{Generation of Transgene free PD patient specific hiPSCs}

Over decades' studies of PD, the second prevalent neurodegenerative disorder, were primarily relied on the analysis of post mortem of PD brains and mouse models due to the inaccessibility to the live PD patient's brain tissues. To date, it is possible to generate and study the PD relevant cell types in culture by virtue of advanced development of iPSC technology. In the last few years, various monogenic PD hiPS cell lines have been generated (Table 1.2), and provided insights into mechanisms contributing to PD progression, like mitochondrial dysfunction and oxidative stress. However, all of these PD-hiPS cell lines were established using retroviral delivery of reprogramming transgenes. There are several drawbacks for the use of integrating retroviral vectors to generate hiPSCs: multiple random insertions of transgenes into the host genomes would disturb the genomic integrity; exogenous reprogramming genes might not be completely silenced and interfere with the further use during differentiation or therapeutic purpose (Hartfield, Fernandes, Vowles, Cowley, \& Wade-Martins, 2012). 
In this work, a polycistronic lentiviral vector (hSTEMCCA) encoding 4 defined factors (Oct4, Sox2, KIf4, and c-Myc) and flanked one loxP site on 3'UTR (Somers, et al., 2010) was employed to convert PD patients' dermal fibroblasts to pluripotent states, and subsequently excised following reprogramming. In consequence of lentiviral based delivery, the efficiency of hiPSC generation $(0.1 \sim 1.5 \%)$ is greatly higher than retroviral based methods $(0.01 \sim 0.25 \%)$ (Table 1.1). Additionally, $\mathrm{Na}$ butyrate was applied to the cells in the first week of reprogramming. As reported (Mali, et al., 2010), Na butyrate, as a Histone deacetylase inhibitor, largely enhanced the efficiency of hiPSC generation (Figure S2). For each sample, up to 30 hiPSC clones could be picked for propagation primarily. Consistent with previous reports of generation of PD patient specific hiPSCs (Park, Zhao, et al., 2008) (Soldner, et al., 2009) (Sanchez-Danes et al., 2012), the reprogramming efficiency varied among different individuals, but not due to the context of PD or donor age (data not shown).

The advantages of the use of a polycistronic hSTEMCCA for hiPSC generation are that it would lead to one insertion site on the host genome, and transgene can be excised via Cre-loxP recombination. Thus, Cre-mediated excision of transgenes was carried out among the generated control/PD-hiPSCs. It was successful to cut off the transgenes from the host genome as confirmed by PCR and southern blotting. In this way, transgene free control/PD-hiPSCs were obtained, characterized for the pluripotency. Moreover, they were further differentiated to PD relevant cell type, midbrain dopaminergic neurons, for study of PD pathogenesis.

Although the risk of exo-transgenic insertion was minimized via Cre excision, it should be noticed that one small DNA fragment ( $200 \mathrm{bp})$ of an inactive viral LTR remains in the host genome. The incomplete elimination might give rise to insertional mutagenesis. Although the possibility of single integration which induced the interruption of gene regulations is statistically minimal, the risk can be reduced by targeting the hSTEMCCA into a safe locus on the genome (Somers, et al., 2010) (Stadtfeld, Maherali, Borkent, \& Hochedlinger, 2010). Furthermore, the non-integrating approach, like RNA based method (Warren et al., 2010), can be applied for reprogramming. However the low efficiency of hiPSC generation is a problem and might be might be improved by additional combinations of treatments with small molecules, like Valproic acid (Shi et al., 2008) or Na butyrate (Mali, et al., 2010). 


\subsection{Derivation of Control/PD-hiPSC Neural Precursors and Transcriptome}

Analysis

To get more insights into the putative molecular players involved in PD etiology and progression, neural precursors were derived from control/PD-hiPSCs and subjected to transcriptome analysis. Neural precursors are multipotent cells, which are able to differentiate further into astrocytes, oligodendrocytes, and neurons. Besides, neural precursors are a homogeneous cell population instead of mixed heterogeneous differentiated neuronal cells. Therefore, it is applicable to perform transcriptome analysis using control/PD-hiPSC derived neural precursors to reduce noise backgrounds, and more importantly to reveal the possible regulatory networks at the very early stage of PD progression.

Herein, derivation of control/PD-hiPSC neural precursors was set up for the combination of stromal feeder co-culture and BMP inhibitor treatment. Following one week of neural induction, it was possible to observe the neural rosette structures. Neural rosette cell is a novel neural stem cell type which holds broad potential toward CNS and PNS lineage differentiation and capability of in vivo functional engraftment in rodent models (Elkabetz, et al., 2008). Therefore, neural rosette structures were manually picked up and expanded to establish the neural precursor cell (NPC) lines. Isolated neural precursors showed the homogeneous population, the proliferative property, and expressed these neural specific markers: Sox1, Pax6, Msi1 and Nestin. Afterwards, a collection of control/PD-hiPSC derived neural precursors were used for profiling of gene expression. According to the heatmap of hierarchical correlation, the discrepancy between clones of the same sample was possible to be observed, and might be due to either clonal variations from the parental hiPSC clone or the process of derivation of neural precursors. However, a recent report has shown that there is a risk of some undifferentiated Oct4 positive cells remaining in the NPC culture (Mak et al., 2012). They suggested that use of magnetic bead sorting with a neural cell adhesion molecule antibody against polysialic acid neural cell adhesion molecule (PSA-NCAM) to enrich more specific and pure population of neural precursors (Mak, et al., 2012). In another study, neural induction of hESC/hiPSC using monolayer culture with treatments of dual SMAD inhibition was developed and showed high efficiency of neural conversion (S. M. 
Chambers, et al., 2009). Both of these studies could reduce the uncertain effects from co-culture with stromal factors and enrich the population of neural precursors.

Through the profiling of gene expression for each derived neural precursor from control and PD-hiPSCs, the transcriptome analysis was set up for comparison of controls vs. PDs. Although a set of differential gene expressions was perceived, the overall gene enrichment groups were diverse (Figure 3.12.A), and differences of gene expression between controls and PDs were not significant. It is probably due to either the variable genetic backgrounds from different individuals or the number of samples (controls or PDs) that was not statistically representative for the major PD population. However, a class of genes involved in axon guidance pathway was noticed. The axon guidance pathway (Figure S1), which regulates the formation of neuronal network by driving axons towards their synaptic targets, has been implicated to predispose to the development of PD. The studies of genetic variability (Single-nucleotide polymorphisms (SNPs)) in the axon guidance pathway associated with the PD have been reported (J. M. Kim, et al., 2011) (Bossers et al., 2009) (Srinivasan et al., 2009). Relying on whole-genome association study of SNPs in PD patients and controls from unaffected siblings, one research group used a bioinformatics approach to discover joint actions of common gene variants within axon guidance pathway that were predictive of PD susceptibility (Lesnick, et al., 2007). Besides, the gene expression analysis in post-mortem brains of 23 PD patients and 23 healthy donors also showed that genes associated with axon-guidance were differentially expressed in the PD-affected brain tissues (Papapetropoulos et al., 2006), further suggesting the relevance of axon guidance pathway in PD. Therefore, as appeared in the transcriptome analysis, some differentially expressed genes (Figure 3.12.B) in the axon guidance pathway might play roles in PD progression. Of note, EPHA4 gene expression was significantly increased (3.5 fold) in affected PD population. EPHA4 has been shown to display high expression level in the degenerated motor neurons in amyotrophic lateral sclerosis (ALS). Inhibition of EPHA4 could rescue neurodegeneration in ALS (Van Hoecke et al., 2012). Thus, EPHA4, or even other candidate genes within axon guidance pathway, might be the target for further investigation of PD pathogenesis.

The here performed transcriptome analysis revealed that 3 genes which were susceptible to Parkinson disease (e.g. GAK, DGKQ, and FGF20) displayed an altered transcription in PD-hiPSC derived neural precursors. Nevertheless, only for FGF20 it is noticed that gene expression was reduced remarkably ( $\sim$ fold) in affected PD population, which was further 
verified by QRT-PCR analysis. FGF20 has been suggested to promote the differentiation of dopaminergic neurons (Correia, Anisimov, Roybon, Li, \& Brundin, 2007) (Shimada, et al., 2009) and prevent the cell death of dopaminergic neurons (Ohmachi, Mikami, Konishi, Miyake, \& Itoh, 2003). More importantly, genetic variability (SNPs) of FGF20 is strongly correlated with the risk of PD (van der Walt, et al., 2004) (Mizuta et al., 2008) (Pan et al., 2012).

Through the transcriptome analysis of control/PD-hiPSC derived neural precursors, the correlation might be established among gene expression profiling of affected PD derived neural precursors and genomic variants studies of PD patients. The previous reports about the risks of genetic variability associated with PD might find out the supportive evidence and even the downstream regulatory networks via gene expression analysis in PD-hiPSC derived neural precursors. Thus, our analysis clearly points to the potential impact of hiPSCs in getting novel insights into the molecular pathways controlling disease mechanisms. The derivation of basically any tissue of the three germ layers from patient's hiPS cells provides useful material for molecular analysis.

\subsection{Direct differentiation of midbrain Dopaminergic (mDA) Neurons from Control/PD-hiPSCs and Phenotypic Assays}

It has long been thought that neurodegeneration in PD reflects the cooperation of genetic susceptibility and environmental vulnerability. In particular, mitochondrial dysfunction and oxidative stress have been implicated in the cell death of mDA neurons in PD. To evaluate the effect of mitochondrial damage and oxidative stress on PD affected neurons, control/PD-hiPSCs were committed to conversion of $\mathrm{mDA}$ neurons using floor plate (FP)-based dopaminergic neuron differentiation. The advantage of FP-based approach is the highly increased amount of differentiated cells displaying a midbrain dopaminergic fate and faithful representation of the PD relevant cell types.

By conducting treatment of CCCP, which interrupts mitochondrial membrane potential (MMP), the expression of mitochondrial protein (TOM20) was monitored and found reduced in PD-hiPSC derived mDA neurons, indicating that mitochondrial activity was attenuated. It showed that PD-hiPSC derived neurons were sensitive to CCCP-induced mitochondrial damage. Besides, examination of CCCP-induced loss of MMP resulting in the mitochondrial 
dysfunction, in the future, it would be necessary to develop more extensive assessments of cellular stress on PD-hiPSC derived mDA neurons, like the various external stimulations described in (Cooper et al., 2012) (Figure 4.1). Thus, it could specify the exact mechanism leading to PD and provide a platform for screening the potential therapeutic targets for PD.

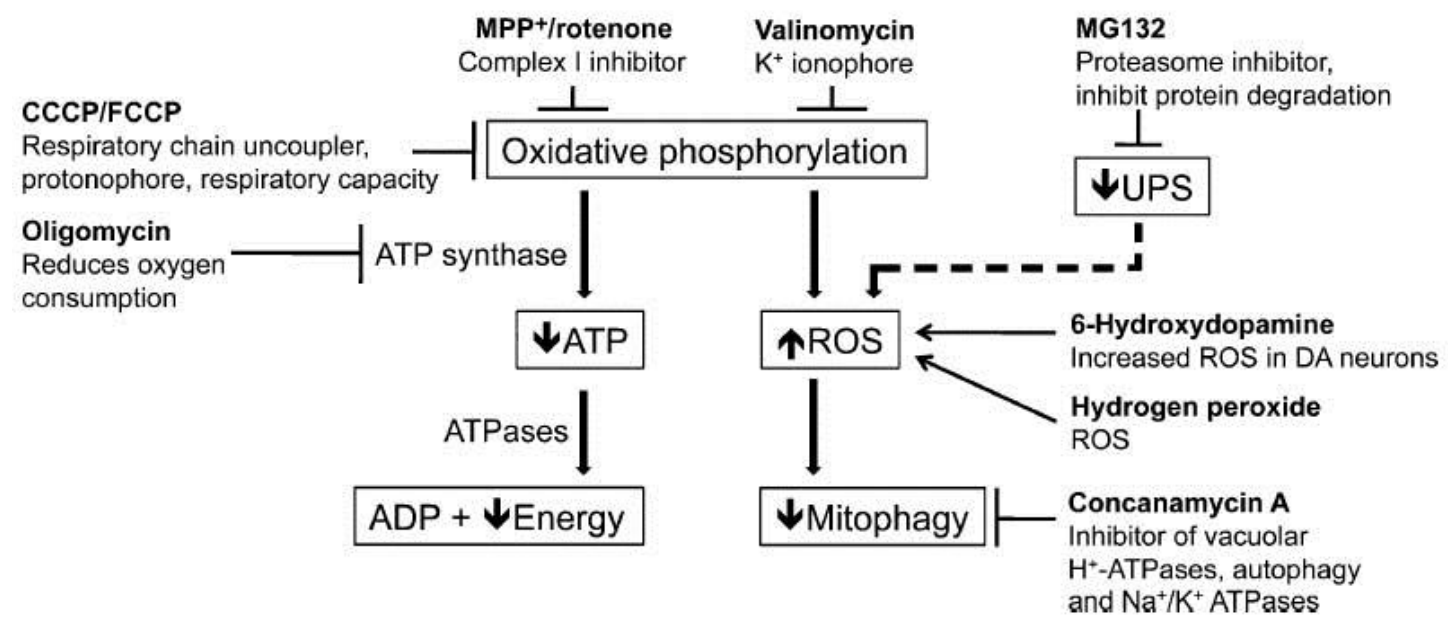

Figure 4.1: Various Cellular Stress Treatments on PD-hiPSC derived neural cells.

Oliver Cooper's group applied different toxins to measure mitochondrial responses, including ROS production, loss of MPP, and depletion of ATP (energy), on PD-hiPSC derived neural cells and their parental fibroblasts. They showed that neural cells were more vulnerable for cell stress as compared to fibroblasts (Cooper, et al., 2012).

\subsection{The Role of FGF20 in PD}

FGF20 is a paracrine growth factor, preferentially expressed in the substantia nigra pars compacta (SNpc) (Itoh \& Ornitz, 2011). FGF20 signaling is mediated through phosphorylation of FGFR1c that is a receptor tyrosine kinase and activates the downstream MAPK pathway (Figure 4.2). Since both of FGF20 and FGFR1c are expressed in most mDA neurons, FGF20 might act in an autocrine/paracrine manner to regulate the differentiation and cell survival of mDA neurons (Itoh \& Ohta, 2013). With neurotrophic activity, FGF20 prevents cell death and thus promotes cell survival of dopaminergic neurons in 6-OHDA injured rodent model (Sleeman, et al., 2012). From our current result of gene expression profiling, the expression of FGF20 was reduced in PD-hiPSC derived neural precursors. Therefore, it is expected that FGF20 might exert a neuroprotective effect on PD affected mDA neurons exposed to 
external toxins. Indeed, pretreatment of PD-hiPSC derived mDA neurons with FGF20 counteracted the cellular stress following either CCCP induced mitochondrial damage (Figure 3.18) or $\mathrm{H}_{2} \mathrm{O}_{2}$ induced excess reactive oxygen species (ROS) (Figure 3.19), which indirectly confirmed that restorative effect of FGF20 in neurodegeneration of PD. In connection with the results of reduced expression in PD-hiPSC derived neural precursors and elicited neuroprotective effect, FGF20 plays a critical role of maintaining normal function of mDA dopaminergic neurons.

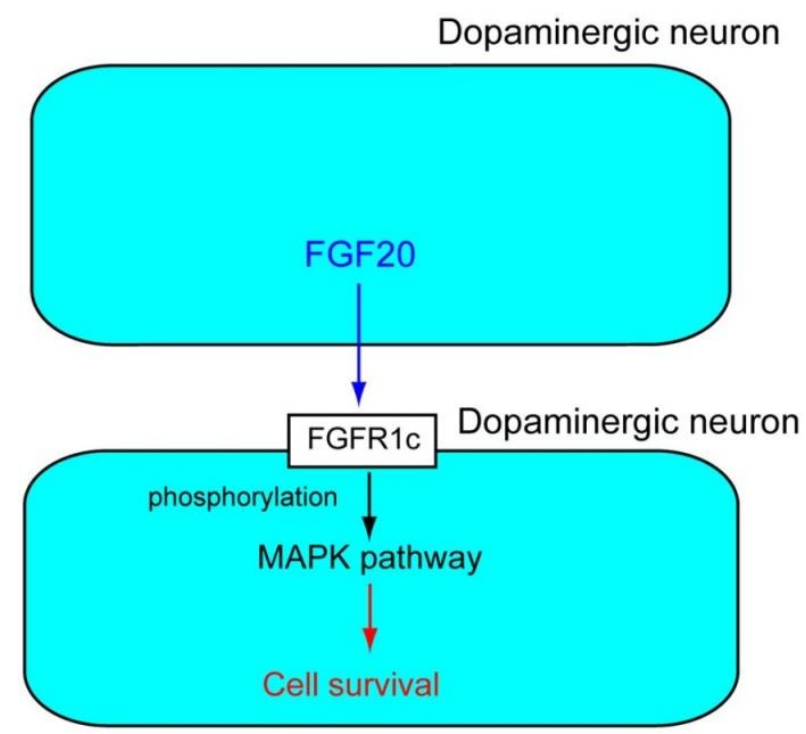

Figure 4.2: The action of FGF20 on dopaminergic neurons.

Acting in a paracrine/autocrine manner, FGF20 binds to FGFR1c and phosphorylates the cytoplasmic tyrosine residues to trigger the activation of the downstream MAPK pathway for enhancement of survival of DA neurons. The picture was taken from (Itoh \& Ohta, 2013). 


\section{Supplementary Figures}

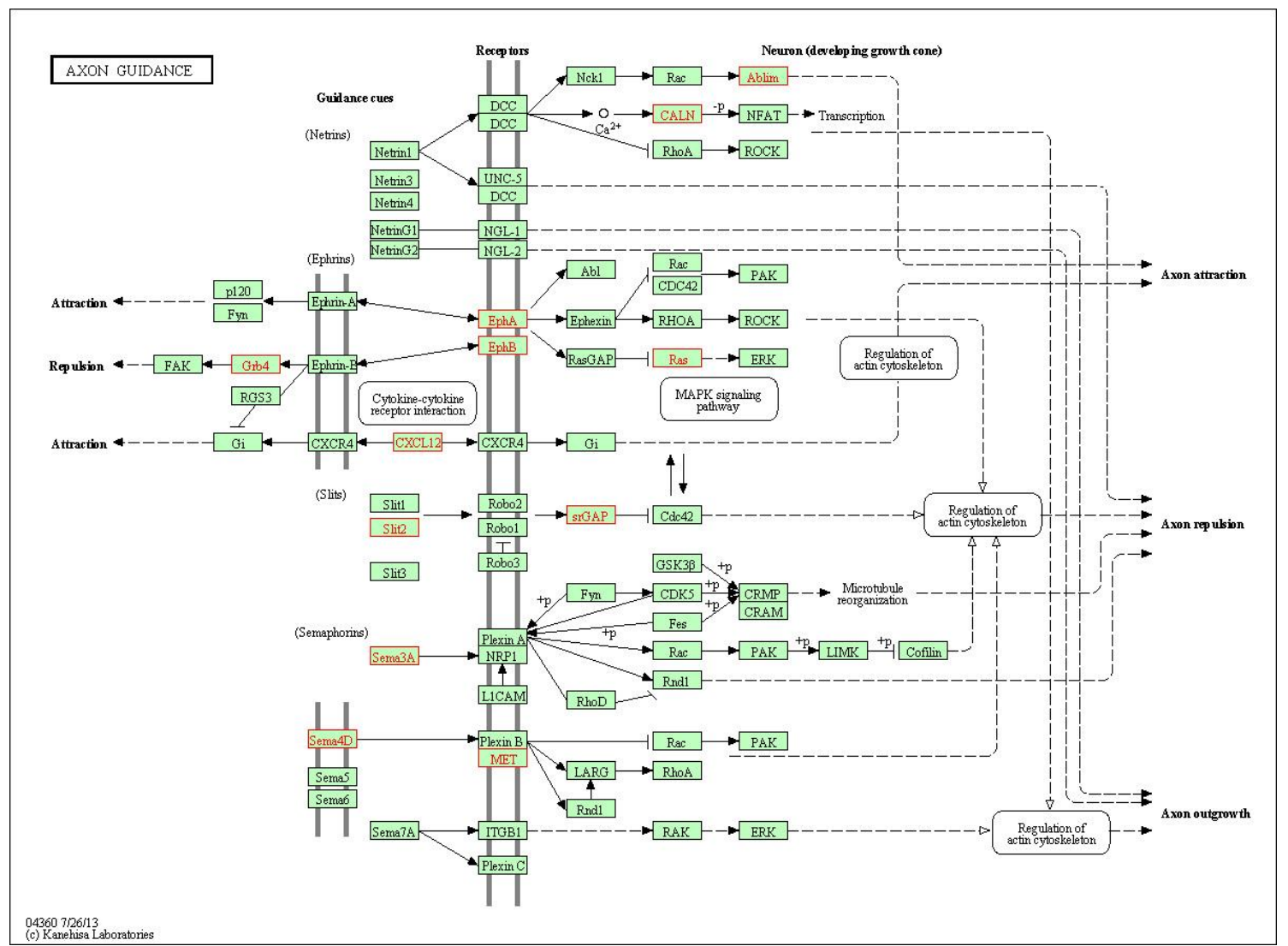

Figure S1: KEGG pathway enrichment analysis of Axon Guidance.

Axon guidance plays a critical role in the formation of neuronal network, concerning how neurons send out axons to reach the corresponding targets. There are four classical families of guidance cues: netrin, ephrin (EPH receptors), Slit, and semaphoring (neuropilins, plexins). Interactions of these signaling networks orientate the growth cone by alterations of cellular cytoskeletal organization.

The changes of gene expression level in the transcriptome analysis of control/PD-hiPSC derived neural precursors are highlighted in red. The picture is adopted from the database of KEGG: Kyoto Encyclopedia of Genes and Genomes (http://www.genome.jp/kegg/). 


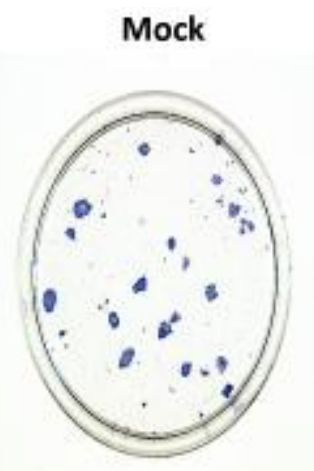

Na Butyrate 7 days

Figure S2: Alkaline Phosphatase (ALP) Staining of hiPSC Colonies.

Representative dishes of ALP staining of hiPSC colonies act in response to treatments of $\mathrm{Na}$ Butyrate during hiPSC generation. 


\section{References}

Aasen, T., Raya, A., Barrero, M. J., Garreta, E., Consiglio, A., Gonzalez, F., et al. (2008). Efficient and rapid generation of induced pluripotent stem cells from human keratinocytes. Nat Biotechnol, 26(11), 1276-1284.

Andersson, E., Tryggvason, U., Deng, Q., Friling, S., Alekseenko, Z., Robert, B., et al. (2006). Identification of intrinsic determinants of midbrain dopamine neurons. Cell, 124(2), 393-405.

Avilion, A. A., Nicolis, S. K., Pevny, L. H., Perez, L., Vivian, N., \& Lovell-Badge, R. (2003). Multipotent cell lineages in early mouse development depend on SOX2 function. Genes Dev, 17(1), 126-140.

Ban, H., Nishishita, N., Fusaki, N., Tabata, T., Saeki, K., Shikamura, M., et al. (2011). Efficient generation of transgene-free human induced pluripotent stem cells (iPSCs) by temperature-sensitive Sendai virus vectors. Proc Natl Acad Sci U S A, 108(34), 14234-14239.

Bekris, L. M., Mata, I. F., \& Zabetian, C. P. (2010). The genetics of Parkinson disease. J Geriatr Psychiatry Neurol, 23(4), 228-242.

Bellin, M., Marchetto, M. C., Gage, F. H., \& Mummery, C. L. (2012). Induced pluripotent stem cells: the new patient? Nat Rev Mol Cell Biol, 13(11), 713-726.

Bennett, M. C., Bishop, J. F., Leng, Y., Chock, P. B., Chase, T. N., \& Mouradian, M. M. (1999). Degradation of alpha-synuclein by proteasome. J Biol Chem, 274(48), 33855-33858.

Blandini, F., \& Armentero, M. T. (2012). Animal models of Parkinson's disease. FEBS J, 279(7), 1156-1166.

Bossers, K., Meerhoff, G., Balesar, R., van Dongen, J. W., Kruse, C. G., Swaab, D. F., et al. (2009). Analysis of gene expression in Parkinson's disease: possible involvement of neurotrophic support and axon guidance in dopaminergic cell death. Brain Pathol, 19(1), 91-107.

Boyer, L. A., Lee, T. I., Cole, M. F., Johnstone, S. E., Levine, S. S., Zucker, J. P., et al. (2005). Core transcriptional regulatory circuitry in human embryonic stem cells. Cell, 122(6), 947-956.

Braak, H., \& Braak, E. (2000). Pathoanatomy of Parkinson's disease. J Neurol, 247 Suppl 2, II3-10.

Byers, B., Cord, B., Nguyen, H. N., Schule, B., Fenno, L., Lee, P. C., et al. (2011). SNCA triplication Parkinson's patient's iPSC-derived DA neurons accumulate alpha-synuclein and are susceptible to oxidative stress. PLoS One, 6(11), e26159.

Byrne, J. A., Pedersen, D. A., Clepper, L. L., Nelson, M., Sanger, W. G., Gokhale, S., et al. (2007). Producing primate embryonic stem cells by somatic cell nuclear transfer. Nature, 450(7169), 497-502.

Caiazzo, M., Dell'Anno, M. T., Dvoretskova, E., Lazarevic, D., Taverna, S., Leo, D., et al. (2011). Direct generation of functional dopaminergic neurons from mouse and human fibroblasts. Nature, 476(7359), 224-227.

Campbell, K. H., McWhir, J., Ritchie, W. A., \& Wilmut, I. (1996). Sheep cloned by nuclear transfer from a cultured cell line. Nature, 380(6569), 64-66.

Chambers, I., Colby, D., Robertson, M., Nichols, J., Lee, S., Tweedie, S., et al. (2003). Functional expression cloning of Nanog, a pluripotency sustaining factor in embryonic stem cells. Cell, 113(5), 643-655. 
Chambers, S. M., Fasano, C. A., Papapetrou, E. P., Tomishima, M., Sadelain, M., \& Studer, L. (2009). Highly efficient neural conversion of human ES and iPS cells by dual inhibition of SMAD signaling. Nat Biotechnol, 27(3), 275-280.

Chen, L., \& Liu, L. (2009). Current progress and prospects of induced pluripotent stem cells. Sci China C Life Sci, 52(7), 622-636.

Ciccone, S., Maiani, E., Bellusci, G., Diederich, M., \& Gonfloni, S. (2013). Parkinson's disease: a complex interplay of mitochondrial DNA alterations and oxidative stress. Int $\mathrm{J} \mathrm{Mol}$ Sci, 14(2), 2388-2409.

Cooper, O., Seo, H., Andrabi, S., Guardia-Laguarta, C., Graziotto, J., Sundberg, M., et al. (2012). Pharmacological rescue of mitochondrial deficits in iPSC-derived neural cells from patients with familial Parkinson's disease. Sci Transl Med, 4(141), 141 ra190.

Correia, A. S., Anisimov, S. V., Roybon, L., Li, J. Y., \& Brundin, P. (2007). Fibroblast growth factor-20 increases the yield of midbrain dopaminergic neurons derived from human embryonic stem cells. Front Neuroanat, 1, 4.

Corti, O., Lesage, S., \& Brice, A. (2011). What genetics tells us about the causes and mechanisms of Parkinson's disease. Physiol Rev, 91(4), 1161-1218.

den Dunnen, W. F. (2013). Neuropathological diagnostic considerations in hyperkinetic movement disorders. Front Neurol, 4, 7.

Denham, M., Bye, C., Leung, J., Conley, B. J., Thompson, L. H., \& Dottori, M. (2012). Glycogen synthase kinase 3 beta and activin/nodal inhibition in human embryonic stem cells induces a pre-neuroepithelial state that is required for specification to a floor plate cell lineage. Stem Cells, 30(11), 2400-2411.

Devine, M. J., Ryten, M., Vodicka, P., Thomson, A. J., Burdon, T., Houlden, H., et al. (2011). Parkinson's disease induced pluripotent stem cells with triplication of the alpha-synuclein locus. Nat Commun, 2, 440.

Elkabetz, Y., Panagiotakos, G., Al Shamy, G., Socci, N. D., Tabar, V., \& Studer, L. (2008). Human ES cell-derived neural rosettes reveal a functionally distinct early neural stem cell stage. Genes Dev, 22(2), 152-165.

Fasano, C. A., Chambers, S. M., Lee, G., Tomishima, M. J., \& Studer, L. (2010). Efficient derivation of functional floor plate tissue from human embryonic stem cells. Cell Stem Cell, 6(4), 336-347.

Fearnley, J. M., \& Lees, A. J. (1991). Ageing and Parkinson's disease: substantia nigra regional selectivity. Brain, 114 ( Pt 5), 2283-2301.

Ferrari, D., Sanchez-Pernaute, R., Lee, H., Studer, L., \& Isacson, O. (2006). Transplanted dopamine neurons derived from primate ES cells preferentially innervate DARPP-32 striatal progenitors within the graft. Eur J Neurosci, 24(7), 1885-1896.

Freberg, C. T., Dahl, J. A., Timoskainen, S., \& Collas, P. (2007). Epigenetic reprogramming of OCT4 and NANOG regulatory regions by embryonal carcinoma cell extract. Mol Biol Cell, 18(5), 1543-1553.

Freire, C., \& Koifman, S. (2012). Pesticide exposure and Parkinson's disease: epidemiological evidence of association. Neurotoxicology, 33(5), 947-971.

Fukui, H., \& Moraes, C. T. (2008). The mitochondrial impairment, oxidative stress and neurodegeneration connection: reality or just an attractive hypothesis? Trends Neurosci, 31(5), 251-256.

Gurdon, J. B. (1962). The developmental capacity of nuclei taken from intestinal epithelium cells of feeding tadpoles. J Embryol Exp Morphol, 10, 622-640. 
Han, S. S., Williams, L. A., \& Eggan, K. C. (2011). Constructing and deconstructing stem cell models of neurological disease. Neuron, 70(4), 626-644.

Hargus, G., Cooper, O., Deleidi, M., Levy, A., Lee, K., Marlow, E., et al. (2010). Differentiated Parkinson patient-derived induced pluripotent stem cells grow in the adult rodent brain and reduce motor asymmetry in Parkinsonian rats. Proc Natl Acad Sci U S A, 107(36), 15921-15926.

Hart, A. H., Hartley, L., Ibrahim, M., \& Robb, L. (2004). Identification, cloning and expression analysis of the pluripotency promoting Nanog genes in mouse and human. Dev Dyn, 230(1), 187-198.

Hartfield, E. M., Fernandes, H. J., Vowles, J., Cowley, S. A., \& Wade-Martins, R. (2012). Cellular reprogramming: a new approach to modelling Parkinson's disease. Biochem Soc Trans, 40(5), 1152-1157.

Hu, K., Yu, J., Suknuntha, K., Tian, S., Montgomery, K., Choi, K. D., et al. (2011). Efficient generation of transgene-free induced pluripotent stem cells from normal and neoplastic bone marrow and cord blood mononuclear cells. Blood, 117(14), e109-119.

Imaizumi, Y., Okada, Y., Akamatsu, W., Koike, M., Kuzumaki, N., Hayakawa, H., et al. (2012). Mitochondrial dysfunction associated with increased oxidative stress and alpha-synuclein accumulation in PARK2 iPSC-derived neurons and postmortem brain tissue. Mol Brain, 5, 35.

Itoh, N., \& Ohta, H. (2013). Roles of FGF20 in dopaminergic neurons and Parkinson's disease. Front Mol Neurosci, 6, 15.

Itoh, N., \& Ornitz, D. M. (2011). Fibroblast growth factors: from molecular evolution to roles in development, metabolism and disease. J Biochem, 149(2), 121-130.

Jackson-Lewis, V., \& Przedborski, S. (2007). Protocol for the MPTP mouse model of Parkinson's disease. Nat Protoc, 2(1), 141-151.

Jankovic, J. (2008). Parkinson's disease: clinical features and diagnosis. J Neurol Neurosurg Psychiatry, 79(4), 368-376.

Jenner, P. (2003). Oxidative stress in Parkinson's disease. Ann Neurol, 53 Suppl 3, S26-36; discussion S36-28.

Jenner, P. (2007). Oxidative stress and Parkinson's disease. Handb Clin Neurol, 83, 507-520.

Jiang, H., Ren, Y., Yuen, E. Y., Zhong, P., Ghaedi, M., Hu, Z., et al. (2012). Parkin controls dopamine utilization in human midbrain dopaminergic neurons derived from induced pluripotent stem cells. Nat Commun, 3, 668.

Kadzik, R. S., \& Morrisey, E. E. (2012). Directing lung endoderm differentiation in pluripotent stem cells. Cell Stem Cell, 10(4), 355-361.

Kaji, K., Norrby, K., Paca, A., Mileikovsky, M., Mohseni, P., \& Woltjen, K. (2009). Virus-free induction of pluripotency and subsequent excision of reprogramming factors. Nature, 458(7239), 771-775.

Kalaitzakis, M. E., Graeber, M. B., Gentleman, S. M., \& Pearce, R. K. (2008). The dorsal motor nucleus of the vagus is not an obligatory trigger site of Parkinson's disease: a critical analysis of alpha-synuclein staging. Neuropathol Appl Neurobiol, 34(3), 284-295.

Kawasaki, H., Mizuseki, K., Nishikawa, S., Kaneko, S., Kuwana, Y., Nakanishi, S., et al. (2000). Induction of midbrain dopaminergic neurons from ES cells by stromal cell-derived inducing activity. Neuron, 28(1), 31-40. 
Kim, D., Kim, C. H., Moon, J. I., Chung, Y. G., Chang, M. Y., Han, B. S., et al. (2009). Generation of human induced pluripotent stem cells by direct delivery of reprogramming proteins. Cell Stem Cell, 4(6), 472-476.

Kim, H., Lee, G., Ganat, Y., Papapetrou, E. P., Lipchina, I., Socci, N. D., et al. (2011). miR-371-3 expression predicts neural differentiation propensity in human pluripotent stem cells. Cell Stem Cell, 8(6), 695-706.

Kim, J. H., Auerbach, J. M., Rodriguez-Gomez, J. A., Velasco, I., Gavin, D., Lumelsky, N., et al. (2002). Dopamine neurons derived from embryonic stem cells function in an animal model of Parkinson's disease. Nature, 418(6893), 50-56.

Kim, J. M., Park, S. K., Yang, J. J., Shin, E. S., Lee, J. Y., Yun, J. Y., et al. (2011). SNPs in axon guidance pathway genes and susceptibility for Parkinson's disease in the Korean population. J Hum Genet, 56(2), 125-129.

Kirkeby, A., Grealish, S., Wolf, D. A., Nelander, J., Wood, J., Lundblad, M., et al. (2012). Generation of regionally specified neural progenitors and functional neurons from human embryonic stem cells under defined conditions. Cell Rep, 1(6), 703-714.

Klein, C., \& Schlossmacher, M. G. (2007). Parkinson disease, 10 years after its genetic revolution: multiple clues to a complex disorder. Neurology, 69(22), 2093-2104.

Kleiner-Fisman, G., Herzog, J., Fisman, D. N., Tamma, F., Lyons, K. E., Pahwa, R., et al. (2006). Subthalamic nucleus deep brain stimulation: summary and meta-analysis of outcomes. Mov Disord, 21 Suppl 14, S290-304.

Kolmakov, K., Belov, V. N., Bierwagen, J., Ringemann, C., Muller, V., Eggeling, C., et al. (2010). Red-emitting rhodamine dyes for fluorescence microscopy and nanoscopy. Chemistry, 16(1), 158-166.

Kriks, S., Shim, J. W., Piao, J., Ganat, Y. M., Wakeman, D. R., Xie, Z., et al. (2011). Dopamine neurons derived from human ES cells efficiently engraft in animal models of Parkinson's disease. Nature, 480(7378), 547-551.

Kurian, L., Sancho-Martinez, I., Nivet, E., Aguirre, A., Moon, K., Pendaries, C., et al. (2013). Conversion of human fibroblasts to angioblast-like progenitor cells. Nat Methods, 10(1), 77-83.

Kurosawa, H. (2007). Methods for inducing embryoid body formation: in vitro differentiation system of embryonic stem cells. J Biosci Bioeng, 103(5), 389-398.

Lang, A. E., \& Lozano, A. M. (1998). Parkinson's disease. Second of two parts. N Engl J Med, 339(16), 1130-1143.

Langston, J. W., Ballard, P., Tetrud, J. W., \& Irwin, I. (1983). Chronic Parkinsonism in humans due to a product of meperidine-analog synthesis. Science, 219(4587), 979-980.

Lee, J. Y., Nagano, Y., Taylor, J. P., Lim, K. L., \& Yao, T. P. (2010). Disease-causing mutations in parkin impair mitochondrial ubiquitination, aggregation, and HDAC6-dependent mitophagy. J Cell Biol, 189(4), 671-679.

Lee, S. H., Lumelsky, N., Studer, L., Auerbach, J. M., \& McKay, R. D. (2000). Efficient generation of midbrain and hindbrain neurons from mouse embryonic stem cells. Nat Biotechnol, 18(6), 675-679.

Lees, A. J. (2007). Unresolved issues relating to the shaking palsy on the celebration of James Parkinson's 250th birthday. Mov Disord, 22 Suppl 17, S327-334.

Lesnick, T. G., Papapetropoulos, S., Mash, D. C., Ffrench-Mullen, J., Shehadeh, L., de Andrade, M., et al. (2007). A genomic pathway approach to a complex disease: axon guidance and Parkinson disease. PLoS Genet, 3(6), e98. 
Li, C., Zhou, J., Shi, G., Ma, Y., Yang, Y., Gu, J., et al. (2009). Pluripotency can be rapidly and efficiently induced in human amniotic fluid-derived cells. Hum Mol Genet, 18(22), 4340-4349.

Lill, C. M., Roehr, J. T., McQueen, M. B., Kavvoura, F. K., Bagade, S., Schjeide, B. M., et al. (2012). Comprehensive research synopsis and systematic meta-analyses in Parkinson's disease genetics: The PDGene database. PLoS Genet, 8(3), e1002548.

Lin, K. H., Oleskevich, S., \& Taschenberger, H. (2011). Presynaptic Ca2+ influx and vesicle exocytosis at the mouse endbulb of Held: a comparison of two auditory nerve terminals. J Physiol, 589(Pt 17), 4301-4320.

Lindvall, O., \& Bjorklund, A. (2004). Cell therapy in Parkinson's disease. NeuroRx, 1(4), 382-393.

Lotharius, J., \& Brundin, P. (2002). Impaired dopamine storage resulting from alpha-synuclein mutations may contribute to the pathogenesis of Parkinson's disease. Hum Mol Genet, 11(20), 2395-2407.

Mak, S. K., Huang, Y. A., Iranmanesh, S., Vangipuram, M., Sundararajan, R., Nguyen, L., et al. (2012). Small molecules greatly improve conversion of human-induced pluripotent stem cells to the neuronal lineage. Stem Cells Int, 2012, 140427.

Mali, P., Chou, B. K., Yen, J., Ye, Z., Zou, J., Dowey, S., et al. (2010). Butyrate greatly enhances derivation of human induced pluripotent stem cells by promoting epigenetic remodeling and the expression of pluripotency-associated genes. Stem Cells, 28(4), 713-720.

Mansouri, A., Fukumitsu, H., Schindehuette, J., \& Krieglstein, K. (2009). Differentiation of embryonic stem cells. Curr Protoc Neurosci, Chapter 3, Unit3 6.

Matsuda, N., Sato, S., Shiba, K., Okatsu, K., Saisho, K., Gautier, C. A., et al. (2010). PINK1 stabilized by mitochondrial depolarization recruits Parkin to damaged mitochondria and activates latent Parkin for mitophagy. J Cell Biol, 189(2), 211-221.

Mizuta, I., Tsunoda, T., Satake, W., Nakabayashi, Y., Watanabe, M., Takeda, A., et al. (2008). Calbindin 1, fibroblast growth factor 20, and alpha-synuclein in sporadic Parkinson's disease. Hum Genet, 124(1), 89-94.

Morizane, A., Doi, D., Kikuchi, T., Nishimura, K., \& Takahashi, J. (2011). Small-molecule inhibitors of bone morphogenic protein and activin/nodal signals promote highly efficient neural induction from human pluripotent stem cells. J Neurosci Res, 89(2), 117-126.

Murry, C. E., \& Keller, G. (2008). Differentiation of embryonic stem cells to clinically relevant populations: lessons from embryonic development. Cell, 132(4), 661-680.

Nakamura, K., Nemani, V. M., Azarbal, F., Skibinski, G., Levy, J. M., Egami, K., et al. (2011). Direct membrane association drives mitochondrial fission by the Parkinson disease-associated protein alpha-synuclein. J Biol Chem, 286(23), 20710-20726.

Narsinh, K. H., Jia, F., Robbins, R. C., Kay, M. A., Longaker, M. T., \& Wu, J. C. (2011). Generation of adult human induced pluripotent stem cells using nonviral minicircle DNA vectors. Nat Protoc, 6(1), 78-88.

Nguyen, H. N., Byers, B., Cord, B., Shcheglovitov, A., Byrne, J., Gujar, P., et al. (2011). LRRK2 mutant iPSC-derived DA neurons demonstrate increased susceptibility to oxidative stress. Cell Stem Cell, 8(3), 267-280.

Nichols, J., Zevnik, B., Anastassiadis, K., Niwa, H., Klewe-Nebenius, D., Chambers, I., et al. (1998). Formation of pluripotent stem cells in the mammalian embryo depends on the POU transcription factor Oct4. Cell, 95(3), 379-391. 
Obeso, J. A., Rodriguez-Oroz, M. C., Goetz, C. G., Marin, C., Kordower, J. H., Rodriguez, M., et al. (2010). Missing pieces in the Parkinson's disease puzzle. Nat Med, 16(6), 653-661.

Ohmachi, S., Mikami, T., Konishi, M., Miyake, A., \& Itoh, N. (2003). Preferential neurotrophic activity of fibroblast growth factor-20 for dopaminergic neurons through fibroblast growth factor receptor-1c. J Neurosci Res, 72(4), 436-443.

Ohmachi, S., Watanabe, Y., Mikami, T., Kusu, N., Ibi, T., Akaike, A., et al. (2000). FGF-20, a novel neurotrophic factor, preferentially expressed in the substantia nigra pars compacta of rat brain. Biochem Biophys Res Commun, 277(2), 355-360.

Okita, K., Ichisaka, T., \& Yamanaka, S. (2007). Generation of germline-competent induced pluripotent stem cells. Nature, 448(7151), 313-317.

Ossowska, K., Smialowska, M., Kuter, K., Wieronska, J., Zieba, B., Wardas, J., et al. (2006). Degeneration of dopaminergic mesocortical neurons and activation of compensatory processes induced by a long-term paraquat administration in rats: implications for Parkinson's disease. Neuroscience, 141(4), 2155-2165.

Pan, J., Li, H., Wang, Y., Ma, J. F., Zhang, J., Wang, G., et al. (2012). Fibroblast growth factor 20 (FGF20) polymorphism is a risk factor for Parkinson's disease in Chinese population. Parkinsonism Relat Disord, 18(5), 629-631.

Pankratz, N., Wilk, J. B., Latourelle, J. C., DeStefano, A. L., Halter, C., Pugh, E. W., et al. (2009). Genomewide association study for susceptibility genes contributing to familial Parkinson disease. Hum Genet, 124(6), 593-605.

Papapetropoulos, S., Ffrench-Mullen, J., McCorquodale, D., Qin, Y., Pablo, J., \& Mash, D. C. (2006). Multiregional gene expression profiling identifies MRPS6 as a possible candidate gene for Parkinson's disease. Gene Expr, 13(3), 205-215.

Park, I. H., Arora, N., Huo, H., Maherali, N., Ahfeldt, T., Shimamura, A., et al. (2008). Disease-specific induced pluripotent stem cells. Cell, 134(5), 877-886.

Park, I. H., Zhao, R., West, J. A., Yabuuchi, A., Huo, H., Ince, T. A., et al. (2008). Reprogramming of human somatic cells to pluripotency with defined factors. Nature, 451(7175), 141-146.

Pawitan, J. A. (2011). Prospect of cell therapy for Parkinson's disease. Anat Cell Biol, 44(4), 256-264.

Poskanzer, D. C., \& Schwab, R. S. (1963). Cohort Analysis of Parkinson's Syndrome: Evidence for a Single Etiology Related to Subclinical Infection About 1920. J Chronic Dis, 16, 961-973.

Pu, J., Jiang, H., Zhang, B., \& Feng, J. (2012). Redefining Parkinson's disease research using induced pluripotent stem cells. Curr Neurol Neurosci Rep, 12(4), 392-398.

Rakovic, A., Shurkewitsch, K., Seibler, P., Grunewald, A., Zanon, A., Hagenah, J., et al. (2013). Phosphatase and tensin homolog (PTEN)-induced putative kinase 1 (PINK1)-dependent ubiquitination of endogenous Parkin attenuates mitophagy: study in human primary fibroblasts and induced pluripotent stem cell-derived neurons. J Biol Chem, 288(4), 2223-2237.

Reinhardt, P., Schmid, B., Burbulla, L. F., Schondorf, D. C., Wagner, L., Glatza, M., et al. (2013). Genetic correction of a LRRK2 mutation in human iPSCs links parkinsonian neurodegeneration to ERK-dependent changes in gene expression. Cell Stem Cell, 12(3), 354-367.

Samii, A., Nutt, J. G., \& Ransom, B. R. (2004). Parkinson's disease. Lancet, 363(9423), 1783-1793. 
Sanchez-Danes, A., Richaud-Patin, Y., Carballo-Carbajal, I., Jimenez-Delgado, S., Caig, C., Mora, S., et al. (2012). Disease-specific phenotypes in dopamine neurons from human iPS-based models of genetic and sporadic Parkinson's disease. EMBO Mol Med, 4(5), 380-395.

Schapira, A. H., \& Jenner, P. (2011). Etiology and pathogenesis of Parkinson's disease. Mov Disord, 26(6), 1049-1055.

Seibler, P., Graziotto, J., Jeong, H., Simunovic, F., Klein, C., \& Krainc, D. (2011). Mitochondrial Parkin recruitment is impaired in neurons derived from mutant PINK1 induced pluripotent stem cells. J Neurosci, 31(16), 5970-5976.

Seki, T., Yuasa, S., Oda, M., Egashira, T., Yae, K., Kusumoto, D., et al. (2010). Generation of induced pluripotent stem cells from human terminally differentiated circulating $T$ cells. Cell Stem Cell, 7(1), 11-14.

Shi, Y., Do, J. T., Desponts, C., Hahm, H. S., Scholer, H. R., \& Ding, S. (2008). A combined chemical and genetic approach for the generation of induced pluripotent stem cells. Cell Stem Cell, 2(6), 525-528.

Shimada, H., Yoshimura, N., Tsuji, A., \& Kunisada, T. (2009). Differentiation of dopaminergic neurons from human embryonic stem cells: modulation of differentiation by FGF-20. J Biosci Bioeng, 107(4), 447-454.

Shimura, H., Hattori, N., Kubo, S., Mizuno, Y., Asakawa, S., Minoshima, S., et al. (2000). Familial Parkinson disease gene product, parkin, is a ubiquitin-protein ligase. Nat Genet, 25(3), 302-305.

Sleeman, I. J., Boshoff, E. L., \& Duty, S. (2012). Fibroblast growth factor-20 protects against dopamine neuron loss in vitro and provides functional protection in the 6-hydroxydopamine-lesioned rat model of Parkinson's disease. Neuropharmacology, 63(7), 1268-1277.

Smith, W. C., \& Harland, R. M. (1992). Expression cloning of noggin, a new dorsalizing factor localized to the Spemann organizer in Xenopus embryos. Cell, 70(5), 829-840.

Soldner, F., Hockemeyer, D., Beard, C., Gao, Q., Bell, G. W., Cook, E. G., et al. (2009). Parkinson's disease patient-derived induced pluripotent stem cells free of viral reprogramming factors. Cell, 136(5), 964-977.

Somers, A., Jean, J. C., Sommer, C. A., Omari, A., Ford, C. C., Mills, J. A., et al. (2010). Generation of transgene-free lung disease-specific human induced pluripotent stem cells using a single excisable lentiviral stem cell cassette. Stem Cells, 28(10), 1728-1740.

Sommer, C. A., Sommer, A. G., Longmire, T. A., Christodoulou, C., Thomas, D. D., Gostissa, M., et al. (2010). Excision of reprogramming transgenes improves the differentiation potential of iPS cells generated with a single excisable vector. Stem Cells, 28(1), 64-74.

Spence, J. R., Mayhew, C. N., Rankin, S. A., Kuhar, M. F., Vallance, J. E., Tolle, K., et al. (2011). Directed differentiation of human pluripotent stem cells into intestinal tissue in vitro. Nature, 470(7332), 105-109.

Spillantini, M. G., Schmidt, M. L., Lee, V. M., Trojanowski, J. Q., Jakes, R., \& Goedert, M. (1997). Alpha-synuclein in Lewy bodies. Nature, 388(6645), 839-840.

Srinivasan, B. S., Doostzadeh, J., Absalan, F., Mohandessi, S., Jalili, R., Bigdeli, S., et al. (2009). Whole genome survey of coding SNPs reveals a reproducible pathway determinant of Parkinson disease. Hum Mutat, 30(2), 228-238. 
Stadtfeld, M., Maherali, N., Borkent, M., \& Hochedlinger, K. (2010). A reprogrammable mouse strain from gene-targeted embryonic stem cells. Nat Methods, 7(1), 53-55.

Studer, L., Psylla, M., Buhler, B., Evtouchenko, L., Vouga, C. M., Leenders, K. L., et al. (1996). Noninvasive dopamine determination by reversed phase HPLC in the medium of free-floating roller tube cultures of rat fetal ventral mesencephalon: a tool to assess dopaminergic tissue prior to grafting. Brain Res Bull, 41(3), 143-150.

Su, Y. C., \& Qi, X. (2013). Inhibition of excessive mitochondrial fission reduced aberrant autophagy and neuronal damage caused by LRRK2 G2019S mutation. Hum Mol Genet.

Tachibana, M., Amato, P., Sparman, M., Gutierrez, N. M., Tippner-Hedges, R., Ma, H., et al. (2013). Human embryonic stem cells derived by somatic cell nuclear transfer. Cell, 153(6), 1228-1238.

Takahashi, K., Tanabe, K., Ohnuki, M., Narita, M., Ichisaka, T., Tomoda, K., et al. (2007). Induction of pluripotent stem cells from adult human fibroblasts by defined factors. Cell, 131(5), 861-872.

Takahashi, K., \& Yamanaka, S. (2006). Induction of pluripotent stem cells from mouse embryonic and adult fibroblast cultures by defined factors. Cell, 126(4), 663-676.

Tamaoki, N., Takahashi, K., Tanaka, T., Ichisaka, T., Aoki, H., Takeda-Kawaguchi, T., et al. (2010). Dental pulp cells for induced pluripotent stem cell banking. J Dent Res, 89(8), 773-778.

Thomas, B., \& Beal, M. F. (2007). Parkinson's disease. Hum Mol Genet, 16 Spec No. 2, R183-194.

Thomson, J. A., Itskovitz-Eldor, J., Shapiro, S. S., Waknitz, M. A., Swiergiel, J. J., Marshall, V. S., et al. (1998). Embryonic stem cell lines derived from human blastocysts. Science, 282(5391), 1145-1147.

Tofaris, G. K., Layfield, R., \& Spillantini, M. G. (2001). alpha-synuclein metabolism and aggregation is linked to ubiquitin-independent degradation by the proteasome. FEBS Lett, 509(1), 22-26.

van der Walt, J. M., Noureddine, M. A., Kittappa, R., Hauser, M. A., Scott, W. K., McKay, R., et al. (2004). Fibroblast growth factor 20 polymorphisms and haplotypes strongly influence risk of Parkinson disease. Am J Hum Genet, 74(6), 1121-1127.

Van Hoecke, A., Schoonaert, L., Lemmens, R., Timmers, M., Staats, K. A., Laird, A. S., et al. (2012). EPHA4 is a disease modifier of amyotrophic lateral sclerosis in animal models and in humans. Nat Med, 18(9), 1418-1422.

Vazin, T., Chen, J., Lee, C. T., Amable, R., \& Freed, W. J. (2008). Assessment of stromal-derived inducing activity in the generation of dopaminergic neurons from human embryonic stem cells. Stem Cells, 26(6), 1517-1525.

Warren, L., Manos, P. D., Ahfeldt, T., Loh, Y. H., Li, H., Lau, F., et al. (2010). Highly efficient reprogramming to pluripotency and directed differentiation of human cells with synthetic modified mRNA. Cell Stem Cell, 7(5), 618-630.

Woltjen, K., Michael, I. P., Mohseni, P., Desai, R., Mileikovsky, M., Hamalainen, R., et al. (2009). piggyBac transposition reprograms fibroblasts to induced pluripotent stem cells. Nature, 458(7239), 766-770.

Xi, J., Liu, Y., Liu, H., Chen, H., Emborg, M. E., \& Zhang, S. C. (2012). Specification of midbrain dopamine neurons from primate pluripotent stem cells. Stem Cells, 30(8), 1655-1663. 
Yu, J., Hu, K., Smuga-Otto, K., Tian, S., Stewart, R., Slukvin, II, et al. (2009). Human induced pluripotent stem cells free of vector and transgene sequences. Science, 324(5928), 797-801.

Yu, J., Vodyanik, M. A., Smuga-Otto, K., Antosiewicz-Bourget, J., Frane, J. L., Tian, S., et al. (2007). Induced pluripotent stem cell lines derived from human somatic cells. Science, 318(5858), 1917-1920.

Zhang, S. C., Wernig, M., Duncan, I. D., Brustle, O., \& Thomson, J. A. (2001). In vitro differentiation of transplantable neural precursors from human embryonic stem cells. Nat Biotechnol, 19(12), 1129-1133.

Zhang, W. Y., de Almeida, P. E., \& Wu, J. C. (2008). Teratoma formation: A tool for monitoring pluripotency in stem cell research StemBook. Cambridge (MA).

Zhou, T., Benda, C., Dunzinger, S., Huang, Y., Ho, J. C., Yang, J., et al. (2012). Generation of human induced pluripotent stem cells from urine samples. Nat Protoc, 7(12), 2080-2089.

Zhou, W., \& Freed, C. R. (2009). Adenoviral gene delivery can reprogram human fibroblasts to induced pluripotent stem cells. Stem Cells, 27(11), 2667-2674.

Zhu, Z., \& Huangfu, D. (2013). Human pluripotent stem cells: an emerging model in developmental biology. Development, 140(4), 705-717. 